University of Northern Colorado

Scholarship \& Creative Works @ Digital UNC

$12-2018$

\title{
Exploring Referee Abuse Through the Lens of the Collegiate Rugby Coach
}

Brittany Lauren Jacobs

Follow this and additional works at: https://digscholarship.unco.edu/dissertations

\section{Recommended Citation}

Jacobs, Brittany Lauren, "Exploring Referee Abuse Through the Lens of the Collegiate Rugby Coach" (2018). Dissertations. 532.

https://digscholarship.unco.edu/dissertations/532

This Text is brought to you for free and open access by the Student Research at Scholarship \& Creative Works @ Digital UNC. It has been accepted for inclusion in Dissertations by an authorized administrator of Scholarship \& Creative Works @ Digital UNC. For more information, please contact Jane.Monson@unco.edu. 
(C)2018

BRITTANY LAUREN JACOBS

ALL RIGHTS RESERVED 


\title{
UNIVERSITY OF NORTHERN COLORADO
}

\author{
Greeley, Colorado
}

The Graduate School

\section{EXPLORING REFEREE ABUSE THROUGH THE LENS OF THE COLLEGIATE RUGBY COACH}

\author{
A Dissertation Submitted in Partial Fulfillment \\ of the Requirements for the Degree of \\ Doctor of Philosophy \\ Brittany Lauren Jacobs
}

College of Natural and Health Sciences School of Sport and Exercise Science Sport Administration

December 2018 
This Dissertation by: Brittany Lauren Jacobs

Entitled: Exploring Referee Abuse through the Lens of the Collegiate Rugby Coach

has been approved as meeting the requirement for the Degree of Doctor of Philosophy in the College of Natural and Health Sciences in the School of Sport and Exercise Science, Sport Administration Program.

Accepted by the Doctoral Committee

Dr. Mark Smith, Ph.D., Committee Co-Chair

Dr. Brent Oja, Ph.D., Committee Co-Chair

Dr. Alan Morse, Ph.D., Committee Member

$\overline{\text { Dr. Milan Larson, Ph.D., Committee Member/Faculty Representative }}$

Date of Dissertation Defense

Accepted by the Graduate School

Linda L. Black, Ed.D.

Associate Provost and Dean

Graduate School and International Admissions 


\begin{abstract}
Jacobs, Brittany. Exploring referee abuse through the lens of the collegiate rugby coach. Published Doctor of Philosophy Dissertation, University of Northern Colorado, 2018.
\end{abstract}

Referees are a valuable contributor to the legitimacy of a sporting contest. Despite this, abuse in sport has become a growing concern and is regularly noted as an obstacle with which referees must contend. Continued abuses have been associated with referee discontinuation and have been noted as a key factor influencing the recruitment and retention of referees. Sporting organizations, coaches, and players all feel the impact when there is an inadequate supply of referees. Though the continuance of the referee is related to the continuance of the sport itself, little research has focused on the referee. Further, the studies that have considered referee abuse have focused only on the perspective of the referee seemingly ignoring the perceptions of other sporting stakeholders. Thus, the purpose of this study was to explore perceptions of referee abuse through the lens of the collegiate rugby coach.

Through a phenomenological research design utilizing semi-structured telephone interviews, the topic of referee abuse in rugby was explored. Purposive sampling allowed for a selection of participants that had adequate knowledge of the phenomenon under investigation. Participants were solicited via USA Rugby utilizing a pre-scripted email provided by the researcher. During the data collection phase, saturation was reached as 15 participants completed two phone interviews. The findings, derived from participant 
data, identified 12 themes that addressed coach perceptions of referee abuse, the multitude of factors that impact perceptions of abuse, and numerous coach-generated solutions to help curtail the abuse of referees.

From these findings, an Ecological Transactional Model of Factors Influencing Referee Abuse is proposed. This model categorizes the factors influencing referee abuse into various ecological levels, beginning with an individual's personal characteristics (the ontological level) and continuing through their cultural existence (the macrosystem). The model suggests that these factors influence both perceptions of referee abuse and the solutions generated regarding referee abuse. The transactional portion of this model also highlights the interaction between various levels of one's environment, proposing that the influences of numerous factors may exist at one time and that each of these factors may also influence one another.

Future research is required to explore referee abuse in other contexts and to illuminate the perceptions of referee abuse through the lenses of additional sporting stakeholders. Recommendations for such research include the application of this proposed model to other sporting contexts, the consideration of generational differences on abuse perceptions, and a further exploration of cultural differences as related to perceptions of abuse. Violence prevention is currently a primary focus in many realms, including the entertainment industry, higher education, and sport. Studies of this nature help to ensure that referee abuse is included in the ongoing dialogue regarding violence prevention in sport. 


\section{ACKNOWLEDGMENTS}

Though the writing of a dissertation may often be considered a solitary act, completion takes a village. To all of those who have been part of my village, I thank you! I must first thank the participants of this study, for without their willingness to share their experiences, none of this would have been possible. Thank you to the Division I-A rugby coaches who endured my onslaught of questions and continual reminder emails. They shared with me personal details and experiences that are the foundation of this work.

Thank you to my family without whom my desire for continued education may not exist. Thank you for supporting me as I pursue my academic dreams and for always expressing your confidence in my ability to succeed. You are the best!

I would also like to thank my amazing wife Kellie for enduring this process alongside me. She has supported me wholeheartedly throughout this entire journey and has been the most welcome distraction. Thank you for believing in me, even when I no longer did, and for pushing me through when I could no longer saw a way forward. I love you! No more degrees for at least another year or two, I promise!

Thank you to my committee members who have never allowed me to settle and have helped me to produce a work that is immensely better as a result of their critiques. Dr. Mark Smith, Dr. Brent Oja, Dr. Alan Morse, and Dr. Milan Larson, I cannot adequately express my gratitude for your time and commitment to this project. Thank you, thank you, thank you! It is my hope that our paths will often cross in the future.

To my fellow Sport Management doctoral students, thank you for always being a shoulder to lean on, for reading my work, for providing a sounding board, and for the laughs. I would not have made it without all of the wonderful memories that we have shared along the way. A big thank you to Nicole and Rammi for taking time out of their 
busy schedules to help me create a codebook and to serve as external coders. You all rock!

Thank you to Dr. Jacob Tingle for serving as an external reviewer for this study. Your feedback and support have been invaluable, and your enthusiasm has been contagious! I feel incredibly privileged by your willingness to assist me throughout this process.

I would be remiss if I did not express my gratitude for all of the professors who have positively impacted my academic career along the way. To all those at the University of New Hampshire and at the University of Texas, you have played an integral role in my academic pursuits and in shaping my teaching philosophy; for this I am eternally grateful.

To my dog, Oliver. I know you can't read, but thank you for forcing me out of the house every day as we went on our afternoon walks. The time to breathe in some fresh air, to stop staring at the computer, and to refresh my mind was paramount to my success in this process. And, for all of those with whom I've shared a smile or a beer in the last four years, thank you for helping me maintain work-life balance during the doctoral process. Those shared moments are priceless. They truly are what life is all about. 


\section{TABLE OF CONTENTS}

\section{CHAPTER}

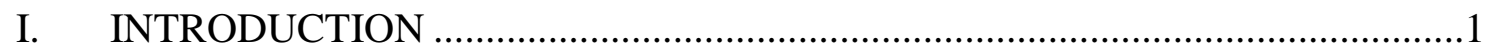

Statement of the Problem............................................................................2

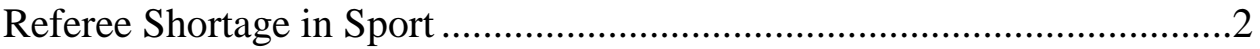

Rugby in America .......................................................................

The Importance of Coaches ...................................................................12

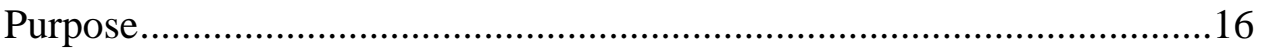

Significance of the Study .............................................................. 17

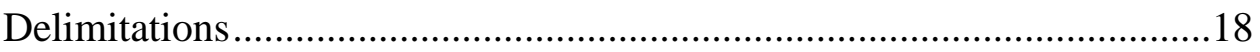

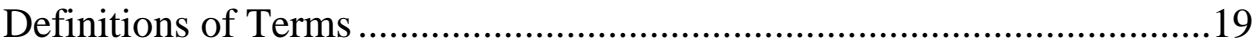

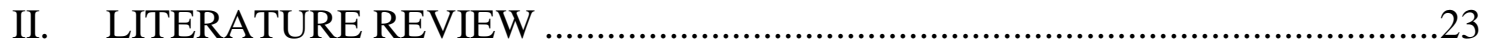

Role of the Referee .........................................................................24

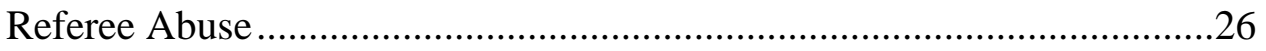

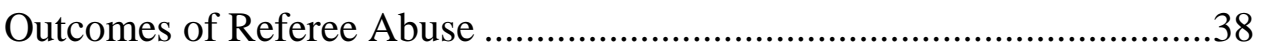

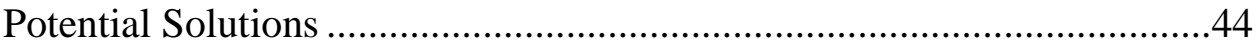

The Role and Power of the Sport Coach..................................................48

Conceptual Framework .................................................................50

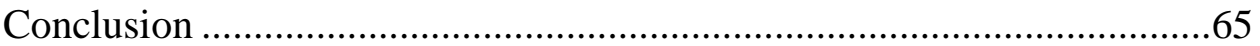

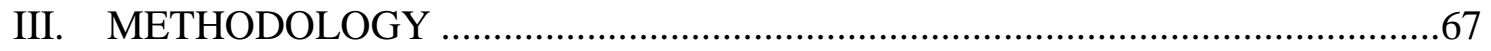

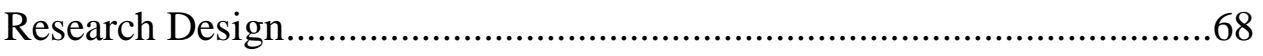

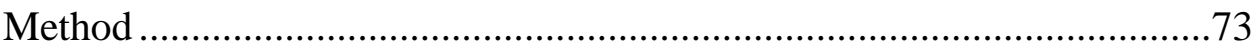

Trustworthiness and Dependability .................................................8

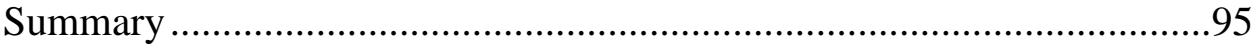

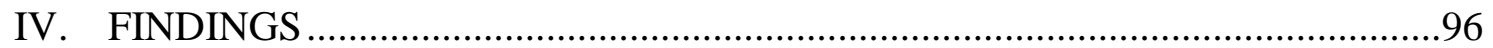

Coaches' Perceptions of Referee Abuse ...................................................96

Factors Influencing Coaches' Perceptions of Referee Abuse .................112

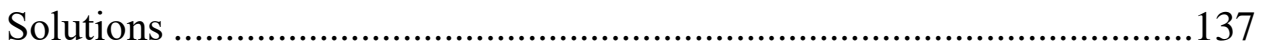

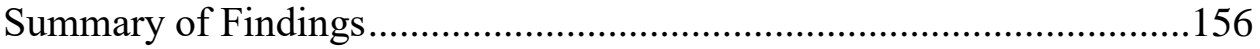




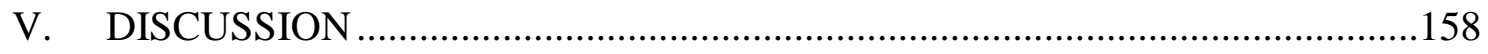

Review of the Research Problem ............................................................158

Perceptions of Referee Abuse …………………………………….......159

Factors Influencing Referee Abuse.......................................................166

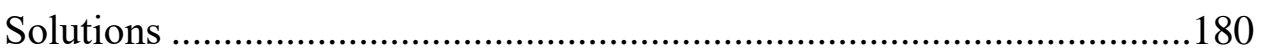

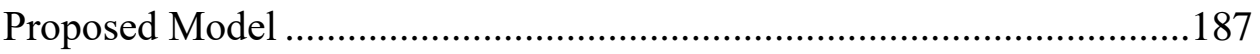

Practical Implications..........................................................................198

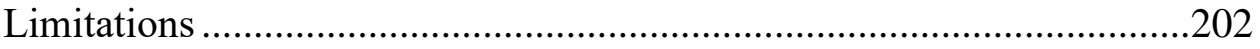

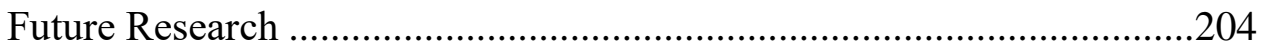

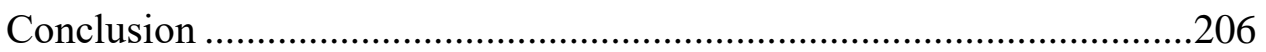

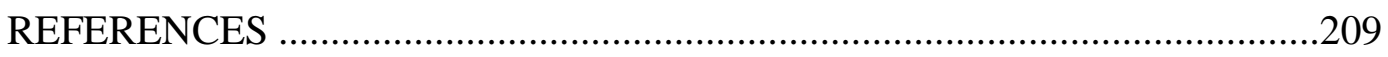

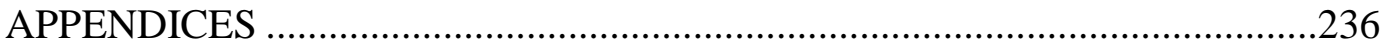

Appendix A: Demographic Questionnaire \& Consent Form....................236

Appendix B: Institutional Review Board Approval.................................238

Appendix C: Participation Solicitation Email to Coaches .........................239

Appendix D: Screening Questionnaire …………………….................240

Appendix E: Consent Form ..............................................................24

Appendix F: Interview Guide I ............................................................24

Appendix G: Interview Guide II ......................................................24 


\section{LIST OF FIGURES}

Figure 1. Cicchetti \& Lynch's (1993) Ecological Transactional Model. .........................54

Figure 2. Stirling \& Kerr's (2014) model of abuse in the coach/athlete relationship........56

Figure 3. Conceptualization of referee abuse from the perspective of the rugby coach....59

Figure 4. Crotty's (1998) model for qualitative research design................................68

Figure 5. Ecological Transactional Model of Factors Influencing Referee Abuse..........189 


\section{CHAPTER I}

\section{INTRODUCTION}

Chapter I seeks to introduce the issue of referee abuse in rugby and highlight the value of exploring the concept from the perspective of a collegiate rugby coach. This chapter is divided into nine sections: (1) Statement of the problem, (2) Referee shortage in sport, (3) Factors contributing to referee attrition, (4) Referee abuse, (5) Rugby in America, (6) Importance of coaches, (7) Purpose, (8) Delimitations, (9) Definition of terms. The introduction begins with a statement of the problem, which is followed by an exploration of referee scarcity. The subsequent section addresses how the apparent shortage of referees demands the exploration of factors contributing to this scarcity, including referee abuse. Chapter I then narrows the scope of the study, exploring the unique historical underpinnings of American rugby, addressing referee abuse in American rugby and emphasizing the importance of coaches in sport, specifically those in collegiate rugby. The preceding sections converge to illuminate the purpose of this study, which is to explore collegiate rugby coaches' perceptions of referee abuse. The chapter concludes with an expression of potential delimitations and an identification of terminology that will be utilized herein. 


\section{Statement of the Problem}

Referee recruitment and retention have become an increasingly pressing issue "for the mere survival of many sporting leagues" (Phillips \& Fairley, 2014, p. 185). To date, research has focused on the many factors that contribute to referee dropout, including referee abuse (e.g., Johnson, 2009; Rayner, Webb, \& Webb, 2016). Despite the developing consensus that referee abuse has become more frequent and more acceptable (Ackery, Tator, \& Snider, 2012; Dell, Gervis, \& Rhind, 2016), few scholars have focused on the root causes of such abuse behaviors or the power of non-referees to impact change. Coaches are viewed as both powerful and influential within the sporting sphere (Jones, Armour, \& Potrac, 2002). Coaches have the power to influence the behaviors of players and spectators, thus developing the competition environment in rugby. As such, this study will seek to explore perceptions and antecedents of referee abuse through the lens of collegiate rugby coaches and to explore possible actions derived from coaches, which may help eliminate or reduce referee abuse.

\section{Referee Shortage in Sport}

Within the sporting structure, referees serve as the arbiters of a match, ensuring fair and safe play. In nearly every organized sport, an official is utilized in some capacity, though their titles may oscillate among official, umpire, and referee. Often, the inclusion of a referee in a sporting competition formalizes the endeavor, differentiating it from recreational play. "Through their engagement in sporting contests and their authority to influence the course of a game, sports officials directly influence, positively or negatively, the quality of the sporting experience both for players and spectators" (Cuskelly \& Hoye, 2013, p. 452). As such, referees are vital to the production of 
organized sport competition, and yet, in recent years, there has been a dearth of research focusing on these important personnel (Warner, Tingle, \& Kellett, 2013). The few studies that have focused directly on the referee suggest a worrying dropout rate compounded by a stressful work environment (e.g., Anshel, Kang, \& Jubenville, 2013; Dorsch \& Paskevich, 2007; Rainey, 1995; Voight, 2009). In fact, the American Sport Education Program (ASEP) has labeled the shortage of officials in the United States a national crisis, and Cuskelly and colleagues (2004) found that it is a challenge to meet the demand for qualified referees across all sports, particularly at the grassroots level. Corroborating these studies, a National Organization of Sports Officials (NASO) survey found that $90 \%$ of high-school association executive directors noted a referee shortage within their own state (Johnson, 2009). Further, the National Federation of High Schools (NFHS) asserted that "for the past several years, state association leaders have warned that we were about to face a crisis relative to the number of officials we have. The crisis is now upon us and it has reached epidemic proportions" (Pappas, 2016, p. 1).

Referee attrition has been seen in a number of sporting contexts, including American rugby. Though nearly 1,000 new referees are trained through USA Rugby's certification programs annually, with 1,012 new referees trained in 2016 (USA Rugby, n.d.), the total number of referees registered with USA Rugby has decreased within the last five years. As of 2012, there were 1,639 registered referees, and at the close of the 2017 membership cycle, USA Rugby listed 1,585 registered referees (L. Zugschwert, personal communication, October 17, 2017). These membership figures show that over the course of the last five years, an overall negative change in referee membership has occurred, with the number of registered referees decreasing by 54 despite a steady flow 
of newly-trained referees joining USA Rugby. The decrease in referee membership is indicative of a significant issue for USA Rugby, as referees are a necessity for the continuation of the sport. According to its strategic plan, USA Rugby is seeking to expand its officiating membership to 2,600 by 2024 , a nearly $40 \%$ increase. Based on the aforementioned decrease in total referee numbers in the last five years, meeting this goal will require significant intervention.

\section{Impact of the Referee Shortage}

High levels of referee attrition create a significant human resource problem for sporting organizations. Additionally, "the cost of referees leaving the game can be significant and wide ranging" (Dell et al., p. 111). The individual referee, the sport organization, and the game itself can all be impacted as referees discontinue participation. Costs related to the individual may include loss of personal income and connections to a sport/activity. Costs to the sporting organization include the loss of resources invested in referee "training, [loss of] organizational knowledge and reputational damage, as well as to the game overall- through leagues closing down due to a lack of available referees" (Dell et al., 2016, p. 111). Ultimately, organized sporting competition is heavily dependent upon referees, and continued attrition impacts the availability and quality of sporting contests (Phillips \& Fairley, 2014).

While league disbandment is not unheard of, a more common result of referee shortages is modified schedules, including adding obscure game times or changes to the days of the week that games are played (Ridinger, 2015). Modification of game schedules is one attempt to alleviate some of the burdens organizations are facing when they lack an adequate number of referees. By modifying schedules, sport organizations utilize the 
same referee for multiple games in one day or for multiple days within one week, leading to an increased workload. In Australia, many officials are refereeing more than five times a week, with a larger workload during tournaments or carnivals (Cuskelly, Evans, \& Hoye, 2004). In the United States, a shortage of officials often requires sports to be played multiple days per week, increasing the workload of the officials to accommodate these matches. In areas where the referee shortages are more severe, sport organizations have been forced to cancel games or to decrease the number of referees involved in each match (Ridinger, Kim, Warner, \& Tingle, 2017; Topp, 2001). For example, a rugby match that typically operates with three total referees, one center official, and two touch judges (sideline officials) may now be assigned a single referee. Referees are not being set up for success when overworked and put into situations with a 1:30 referee-to-player ratio. Ironically, the referee shortage may be one of many factors that prompts referee attrition and impacts organizational outputs.

\section{Factors Contributing to Referee Attrition}

Referee attrition has been linked to numerous factors, including lack of training, lack of a referee community, and problematic social interactions (Warner et al., 2013). Because referees are not often prioritized within the sporting structure, they frequently lack the necessary training and/or mentoring to excel (Auger, Fortier, Thibault, \& Gravelle, 2010; Livingston \& Forbes, 2007). Typically, referee training focuses on rule knowledge and technical pieces of the role, such as positioning, but neglects to teach referees how to deal with more severe problems they may encounter in the role, including referee abuse. Another referee training issue is the lack of professional development, as little continuing education is available to referees throughout their careers (Warner et al., 
2013). Referee recruitment and retention are also influenced by the lack of a referee community (Kellett \& Warner, 2011). It has been suggested that when referees feel unsupported and alone, they are more likely to discontinue participation (Warner et al., 2013). Without appropriate training or support from an officiating community, many officials feel underprepared for the abuses that they will endure as an official. Thus, problematic social interactions, including verbal and physical abuses from players, coaches, and fans, are also associated with referee attrition (Dell et al., 2016). For many, the abuse endured is one of the factors that leads to their discontinuation of the refereeing profession (Anshel \& Weinberg, 1995; Rainey, 1995; Warner et al., 2013).

\section{Referee Abuse}

Referee abuse has a longstanding history in sport. Documentation of referee abuse dates back to the late 1800 s when referees were introduced to formalized sporting competitions. One early account of abuse was recorded in 1903: "The spectators, under the excitement of a great game, become hoodlums, exhibiting violent partisanship and gross profanity. ... Heaping abuse upon the referee, restrained oftentimes only by the players themselves from inflicting upon him actual bodily injury" (Patrick, 1903, p. 106). In 1964, one of the first U.S. court cases involving referee abuse was heard. In Toone v. Adams, a baseball umpire was assaulted after leaving the game (Chiafullo, 1998). As an outcome of the case, the court found that physical abuse should not have a place in sport but agreed that verbal abuse should be considered "a goodly part of the sport" (Chiafullo, 1998, p. 217).

There is emerging consensus that referee abuse is becoming both more frequent and more socially acceptable (Ackery et al., 2012; Dell et al., 2016). Chants such as "Get a rope, get a tree...Hang the f\#@!\&\%g referee" (Chiafullo, 1998, p. 201) have become 
commonplace. The example demonstrates a sporting culture that may normalize referee intimidation and verbal abuse. The sports media has also highlighted egregious occurrences of referee abuse in recent years. For example, in 2016, an Argentinian club soccer referee was shot after red-carding a player. The enraged player went to his bag, produced a gun, and subsequently shot the referee three times - in the head, neck, and chest. The referee did not survive the player's outburst (Perez, 2016). Extreme cases of referee abuse are not limited to soccer-crazed nations like Argentina, nor are they limited to the sport of soccer. In early 2017, a Michigan soccer referee was killed. In this instance, the referee, Kyle Bieniewicz, was preparing to send a player off. Witnesses say that the game had been officiated fairly and that nothing had been done to provoke an attack. Despite this, as Beiniewicz was looking down to retrieve his card, the offending player punched him in the head. The referee immediately hit the ground and turned blue; he had stopped breathing. After being rushed to the hospital, he was pronounced brain dead and was later taken off life support (Anderson, 2017). A similar incident took place about a month later in France as a French rugby player punched a referee in the face after being red-carded. Compared to the instances of referee abuse discussed above, this referee, Benjamin Casty, was fortunate. He was not killed by the attack but instead suffered cheekbone and jaw injuries and was expected to fully recover (Terrell, 2017). With a growing number of examples of referee abuse in various settings, it is not surprising that many are hesitant to take up refereeing as a vocation and that those already involved in officiating often drop out because of the abuse they endure (e.g., Dell et al., 2016; Nazarudin, Omar-Fauzee, \& Din, 2009). 
Rugby is not immune from the issue of referee abuse. Rayner et al. (2016) found that referees in the Rugby Football Union were subjected to significant levels of abuse from players, coaches, and spectators; further, they found that $67 \%$ of rugby referees reported experiencing abuse during their careers. Similarly, a report prepared for the Australian Sports Commission indicated that $78 \%$ of respondents had witnessed abuse of referees across a number of sports (Cuskelly et al., 2004). Cuskelly and colleagues (2004) established that some associations reported a first-year attrition rate of officials as high as $60 \%$. Further, the National Association of Sports Officials (NASO) reported that at least one-third of referees quit after their first year (Sabaini, 2001). Pappas (2016) noted as follows:

The writing has been on the wall for quite some time, but little has been done to truly heed the warning and to address the problem - the "why" of where we are and the "how" to fix it. Everyone has known [referee abuse and subsequent referee dropout] is a problem but what have we done institutionally to solve the problem at the statewide level, within each school, within each local officials' association and each of us individually? (p. 1)

Referees deserve protection from abusive people within the context of a sporting competition (Brackenridge, Pitchford, \& Wilson, 2011), and this systematic issue of referee abuse warrants further study.

\section{Rugby in America}

The sport of rugby is said to have originated at the Rugby School in England, where in 1823 a student playing football [soccer] picked up that ball and ran with it. The disregard for the traditional rules of football led to a new code of the sport, later named rugby after the school where it originated (Collins, 2009). While there was little evidence that confirmed the identity of the schoolboy who created rugby, the rugby community has appointed William Webb Ellis to fill this void. Though it could not have been known at 
the time, the Rugby School would play an integral role in rugby's development within Britain and the world, inextricably linking rugby with the traditions of morality put forth in Muscular Christianity.

The Rugby School prided itself in developing boys with character and governing the school by three notions: religious and moral principle, gentlemanly conduct, and intellectual ability (Collins, 2009). Thomas Arnold, the headmaster at the time of rugby's creation, expanded the goals of the school to include participation in physical activity, thus ascribing a set of moral values to the sport of rugby. This prescription provided meaning for the sport in contexts beyond the school, tying it directly to the Muscular Christianity movement. Muscular Christians believed that sport had the power to instill young men with positive traits, utilizing manly competition to stave off unwanted femininity (Watson, Weir, \& Friend, 2005). With the writing of Tom Hughes' novel Tom Brown's School Days, rugby would soon become an ideology intertwined with Muscular Christianity. In 1857, Hughes' novel highlighted the values of Muscular Christianity in building character for students within the public school system in England (Mangan \& Walvin, 1987). Drawing on his own experiences at the Rugby School, Hughes included an account of a rugby match in his writing, advocating that to play rugby was a way to build on the moral traditions that had been created at the Rugby School. Playing rugby was an ode to moral welfare (Hughes, 1895). The popularity of Hughes' novel and the ideas it encompassed led to numerous other public schools adopting the Rugby tradition (Mangan \& Walvin, 1987). Thus, the moral tradition of Muscular Christianity had now come to include the sport of rugby. 
Stemming from the prolific success of Hughes's novel, rugby grew rapidly in popularity and became known as an establishment sport for men and boys in England. Resulting from the moral foundations of the gentile class that played the sport, rugby later became known as a gentleman's sport, with a firm focus on the laws of the game and sportsmanship. Rugby's reach continued to expand around the world, and in North America, the rugby code was first taken to Canada by students of McGill University who had been introduced to the game in Britain (Collins, 2009). In 1874, the McGill team captain challenged Harvard University's football team to two matches—one under Harvard's football rules and the other under the Rugby School's football rules (Collins, 2009). The Harvard football team was so taken by rugby that they abandoned their own rules, and thus, traditions of rugby in the United States began with collegiate play (USA Rugby, n.d.). Harvard's infatuation with rugby led to the expansion of the game throughout the college sporting community. Rugby flourished in the United States as the sport's ideology exemplified Muscular Christianity, which, as noted above, sought to interlock Christian ideals and physical activity.

The philosophical and moral foundations of rugby make the sport unique from other American sports, creating an expectation of superior moral behavior. Rugby stakeholders have continued to refer to the sport as a gentleman's game, signifying that participants might engage in the competition with a high level of civility (Moore, 2000). Notably, it is common for rugby players to address male referees as sir, denoting respect. In the present day, the game is still governed by core values, including integrity, passion, solidarity, discipline, and respect, as outlined by the World Governing Body for Rugby (World Rugby, 2014). The apparent morality that underpins the rugby sporting ethos 
creates a distinctive context in which to study referee abuse and differentiates the sport from others that may be considered.

Though one may expect that the instances of referee abuse in rugby may be reduced based upon the sport's moral presuppositions, staff members at USA Rugby have found that abuse of officials is still a pervasive problem. James Wilber is the National Championships Manager at USA Rugby and oversees all championship competitions, including collegiate championships. Having attended numerous collegiate matches throughout his career, he expressed it this way:

I have witnessed abuse by players, coaches, parents, and even other referees. Though uncommon, at times there are disturbing themes of homophobia and xenophobia. Sadly, I cannot recall more than one instance of "self-policing," where the abuser was addressed by others for their inappropriate remarks. (J. Wilber, personal communication, November 7, 2017)

Wilber's commentary indicated that abuse is present within collegiate rugby and that additional policies may need to be implemented to curb these abusive behaviors since individuals are not taking initiative on their own or executing their own moral imperatives.

Beyond this, determining an appropriate and agreed-upon definition of abuse within rugby has proved difficult. According to Ed Todd, former Referee Department Director at USA Rugby:

One of the obstacles we run into during disciplinary hearing is identifying/defining abuse. Is it abuse when a sideline person (coach, sub, trainer) shouts "that's the worst call I've ever seen," or "my blind mother could call a better game"; what about "you're a f**king cheat" or "you deserve to be shot"? I have witnessed some of these verbal comments over the years. (personal communication, October 31, 2017)

The pervasive nature of referee abuse within the rugby sporting context is supported by USA Rugby's Collegiate Director, Rich Cortez, as he noted that he has seen "incessantly 
strong and profane language [used against referees]" (personal communication, October 31, 2017). He asserts that he has never personally seen a referee shoved or punched, though he has heard of it occurring in rare instances. Interestingly, he also emphasizes that though referee abuse does exist (as he has seen it firsthand), it does not exist to the degree that referees indicate. The belief that referees overstate abuse suggests a disconnect between rugby referees' perceptions of abuse and coaches' perceptions of referee abuse. Thus, studying referee abuse through the lens of the coach becomes even more compelling.

Cortez and Wilber also indicated that administrators and coaches have the power to advent change within the USA Rugby sporting structure. "I think the standards of behavior by some coaches needs improvement," says Cortez, "and administrators and coaches [have the power to improve the situation] by adopting game day protocols and use of Match Administrators to organize rosters, substitutions, technical zones and sideline manners" (personal communication, October 31, 2017). Wilber supported the assertion that coaches are influential figures within rugby, stating:

Coaches also need to understand that the referee officiating your match today is the referee of the future, and that they have the next largest role to play in fostering a supportive environment that generates more referees of higher quality. If these coaches drive every new referee out of the game due to abuse and lack of empathy for the developing referee, we will surely end up with fewer referees and of less quality. (personal communication, November 7, 2017)

\section{The Importance of Coaches}

In addition to the sentiments of USA Rugby employees, there has been a call for future research to consider not only the referee's perspective of abuses but also the perspective of the coach (Walters, Schluter, Stamp, Thomson, \& Payne, 2016). The perspective of the coach is valuable when considering referee abuses, as abuse from 
coaches has been found to be a regular occurrence. According to Anshel and Weinberg (1995, 1996), abuse from coaches is the most common and the second-most severe source of stress for basketball officials. The notion that coaches regularly act as the perpetrators of referee abuse has been further supported in the literature (e.g., Anshel \& Weinberg, 1995, 1996; Nazarudin et al., 2009; Rayner et al., 2016). Because coaches are one of the major propagators of referee abuse, it is important to unpack their experiences with referee abuse and to understand the factors that contribute to such behaviors.

Despite the regular display of their own poor behavior, coaches have also been found to take responsibility for the "development of moral, respectful, and pro-social behaviors" (Vella, Oades, \& Crowe, 2011, p. 43) of their players. In doing so, they place strong emphasis on respect for teammates, opposition, and officials (Vella et al., 2011). Though coaches may intend to teach their players positive sporting behaviors, players are likely to normalize and mimic the abusive behaviors of their coaches regardless of the coach's intention (Fields, Collins, \& Comstock, 2007). Players view their coaches as role models and experts (Conroy \& Coatsworth, 2006; Raakman, Dorsch, \& Rhind, 2010) and thus are likely to simulate the behaviors of their coach, even if such behaviors are incongruous with character-development teachings.

At the collegiate level, the influence of coaches becomes even more pronounced. In the college setting, student athletes are no longer controlled by parental oversight, enhancing the coach's power and influence over their players. With such a large influence on players, coaches have the power to perpetuate a vicious cycle of referee abuse or to address and mitigate the issue. Beyond the coaches' influence within the sporting structure, collegiate coaches were selected as participants for this study as 
college rugby is the largest segment of the rugby-playing population. Within USA Rugby, collegiate rugby makes up the largest percentage of the total player membership, with 34,004 registered members accounting for $31.2 \%$ of the total player membership and $29 \%$ of the overall membership of USA Rugby (Top Quartile Performance Institute, 2016). As a result, there are more registered collegiate rugby coaches than there are coaches in any other segment of the game (Top Quartile Performance Institute, 2016). The sheer number of collegiate rugby coaches places them in a position of power within the USA Rugby organization and within many of the decision-making committees. In rugby, the men's collegiate game is not governed by the National Collegiate Athletic Association; instead, it is governed directly by USA Rugby. This unique sporting structure differentiates rugby from nearly every other collegiate team sporting competition within the United States. Selecting a sporting competition that is not impeded by various levels of organizational hierarchy creates an opportunity for the application of solutions garnered within this research. While many sporting organizations would be required to seek the approval and buy-in of the National Collegiate Athletic Association, USA Rugby has exclusive rights over policy or programmatic change. As such, collegiate rugby becomes an amenable context to explore referee abuse and potential solutions.

Further, because USA Rugby oversees the governance of men's Division I-A (DIA), Division I-AA (DI-AA), and Division II (DII) teams, there is responsibility for the competition structures, national championships, disciplinary policies, eligibility rules, and certification for both coaches and referees (USA Rugby, n.d.). Under USA Rugby's purview, to coach at the collegiate level an individual must do the following: 
1. Register as a coach with USA Rugby. This means that the coach has paid their annual registration fee with USA Rugby.

2. Pass a biennial background screening. This requirement helps to protect the safety of the athletes who would be under the coach's purview.

3. Complete the player protection package. To satisfy this requirement the coach must complete a concussion training and the United States Olympic Committee (USOC) SafeSport training. The concussion training has increased in importance over the last few years as a result of the safety concerns that have been addressed in sports like football. The SafeSport program is intended to help protect athletes from sexual abuse within the sporting context.

4. Complete a certification course. Completing the Level 100 coaching certification will allow a coach to be current within USA Rugby's registration system. The Level 100 certification can be completed online and is nearly identical for every governing body that falls under the auspices of World Rugby. However, in the United States this certification is insufficient if the coach will be coaching contact rugby. In order to coach contact rugby, the coach must complete the second course in the series, a Level 200 Coaching certification.

5. Annually maintain current status. According to USA Rugby, maintaining a current status means that the coach has completed 10 professional development credits annually. Opportunities to complete the professional development credits primarily include officiating or strength \& conditioning certification courses through USA rugby, or related courses offered by the National Federation of High Schools (NFHS) or ASEP. (USA Rugby Coaches, n.d.)

As a result of the coaching requirements set forth by USA Rugby, every collegiate coach must have a base level of knowledge prior to participating in a USA Rugby-sanctioned collegiate competition. This structure again places collegiate rugby in a unique realm of sport. In this instance, the governing body of the sport manages both the sporting competitions and the certification processes for coaches and referees. The oversight of both organizational functions allows USA Rugby to integrate information derived from the collegiate competition into their certification courses, ultimately shaping future collegiate competition.

Though collegiate rugby differs from most other team sports in both governance and structure, the Division I-A level is still synonymous with the highest tier of competition and professionalism within the collegiate rugby game. Seventy teams 
participate in the DI-A men's competition, with 12 teams qualifying for the post-season playoff structure (Division I-A Rugby, n.d.). At the Division I-A level, the competitions are sponsored, most recently by IMG and provide revenue potential to USA Rugby (Solomon, 2014). The top-level collegiate competitions also garner significant television contracts. Such deals include the recent renewal of a contract with CBS Sports Network to televise the Men's Collegiate DI-A Championships in 2018 and 2019 (USA Rugby, n.d.). As in other sports, television coverage helps to ensure that the behaviors modeled within the highest tier of competition often trickle down into lower levels of the sport. Thus, Division I-A teams and their collegiate coaches may have an outsized influence on rugby sporting behaviors. Together with their players, collegiate coaches at the Division I-A level have the power to set the standard of behavior in collegiate rugby. Further, USA Rugby's governance structure allows for the implementation of such standards.

Therefore, it is important to understand referee abuse, its causes, and potential solutions from this perspective.

\section{Purpose}

The purpose of this study was to consider how collegiate rugby coaches perceive referee abuse and what factors have contributed to these perceptions. Thus far, research has primarily considered referee abuse from the perspective of the referee (Rayner et al., 2016) as it relates to their willingness to continue officiating. Therefore, the central purpose of this study was to expand the understanding of referee abuse in the rugby sporting context. As a result, the study explored collegiate rugby coaches' perceptions of referee abuse within the rugby sporting structure to understand the foundation of these 
perceptions. Additionally, this study sought to explore the ways coaches believe referee abuse in the sport could be minimized or eliminated.

Through the exploration of coaches' perceptions and proposed resolutions, sport managers may be able to better understand how referee abuse is perceived in the larger coaching community and look to develop potential strategies to alleviate the problem of coach-related referee abuse. Further, the research findings provided a foundation for policy development, allowing sport managers to take proactive steps toward eliminating referee abuse. As such, this study was guided by the following research questions:

Q1 How do men's Division I-A collegiate rugby coaches perceive referee abuse within the U.S. rugby sporting structure?

Q2 What factors contribute to Division I-A collegiate rugby coaches' perceptions of referee abuse?

Q3 From the perspective of a men's collegiate rugby coach, what actions may alleviate the problem of referee abuse in the college arena?

\section{Significance of the Study}

As previously suggested, high attrition rates may result from referee abuse (Gencay, 2009; Kellett \& Warner, 2011; Stevenson, 2001). Dell and colleagues (2016) have expressed that "abuse exposed officials to occupational stress related to their roles as referees and caused them to question their continued involvement in the game" (p. 117). Though much research has shown that referee abuse is a perpetual problem that relates to referee dropout, recent research has focused on administrative shortcomings and community structures (e.g., Kellett \& Warner, 2011; Warner et al., 2013; Warner, Tingle, \& Kellett, 2012). Despite these limited efforts, referee abuse persists, and further investigation was warranted. Additionally, most officiating research has focused solely on the referee; thus, this study sought to fill a gap in the literature by illuminating the 
issue of referee abuse from an external perspective. Although coaches are one of the main perpetrators of referee abuse (e.g., Friman, Nyberg, Archer, \& Norlander, 2004; Nazarudin et al., 2009; Rayner et al., 2016), they are seen as powerful components in sporting structures. Because the perspective of coaches is highly valued, they were able to help shed light on the otherwise overlooked issue of referee abuse. Understanding referee abuse from the perspective of the coach has helped to paint a more universal picture of the issue, facilitating the riddance of referee abuse and helping to combat the shortage of sports officials.

\section{Delimitations}

This study was limited in the transferability of findings, as only one specific sport—rugby—was be considered. As the first research of its kind to consider referee abuse from a non-referee perspective, the researcher chose to delve more deeply into the data of one sport rather than provide an audit of a number of sports. Additionally, as with any study seeking to explore a controversial topic, those who choose to participate in this study may have been markedly different from those who chose to exclude themselves from the study (Wiederman, 1999). Despite the potential for bias by self-exclusion, the sample size was sufficient to ensure that data saturation was reached and that the results appropriately represent men's rugby coaches' perceptions of both referee abuse and future strategies to combat said abuse. A final limitation of this study was the potential for social desirability. Because the discussion of abuse may be an uncomfortable topic for some, it was possible that participants may have responded to questions in a way that made them appear favorable to the researcher (Grimm, 2010). To help alleviate this concern, participants were informed that their data would remain confidential. 
Additionally, each participant was a pseudonymn associated with all presented data. Beyond this, all mentioned identifying information was changed or stricken from the record; that included items such as team information or conference information.

\section{Definitions of Terms}

The following terms are defined to ensure clarity of use and consistency throughout this study.

Assistant Referee: Linesman (woman) in the sport of rugby. Assistant referees monitor the touch line, as would a touch judge, but may also report foul play and other infringements to the center referee.

Attrition: Gradually reducing strength or effectiveness of someone or something as a result of sustained pressure. As related to employment, when an employee decides to leave a role and the individual is not replaced.

Breakdown: A period of play that includes a tackle and a potential ruck. At this time both teams are competing for possession of the ball.

Division I-A Rugby Coach: As defined by USA Rugby, Division I-A rugby coaches will include men's rugby coaches whose teams participate in the Division I-A level of competition.

Kiwi: A slang term used to reference individuals from New Zealand.

Laws of the Game: These are the rules of the game of rugby union as defined by the international organizing body, World Rugby.

Official: Also referred to as a referee or umpire, the official is the arbiter of a sporting competition. 
Perception: Organizing and interpreting information to understand a situation or one's environment.

Physical Abuse: Intentional and unwanted injury or trauma caused to another via bodily contact. As it relates to sport, sporting implements become an extension of the individual, thus contacting another with a sporting object (e.g., hockey stick) would be considered physical abuse, so long as such contact is outside the rules of the game.

Referee Recruitment: Referee recruitment is defined as successfully placing a new referee into the officiating role (Cuskelly et al., 2004).

Referee: The arbiter of a sporting competition. This person is tasked with managing the match while upholding the rules of competition and the integrity of the game. Also referred to as the center referee or the head referee.

Referee abuse: “A verbal statement or physical act which implies or threatens physical harm to a referee or a referee's property or equipment. Abuse includes, but is not limited to, the following acts committed on a referee: using foul or abusive language toward a referee, spewing any beverage on a referee's personal property, or verbally threatening a referee." (Chiafullo, 1998, p. 202)

Referee Retention: Retention is considered to be the preservation of an official from one season to another. A retention rate could be produced by dividing the number of officials that have continued into the next season 
by the number of available officials from the close of the previous season (Cuskelly et al., 2004).

Scrum: Used to restart the game, a scrum allows the two teams to contest for the ball. Eight players from each team create a pack that pushes against each another. The ball is rolled between the two packs, and each pack attempts to gain possession for their team by kicking the ball backward to their teammates.

Ruck: A ruck is formed if the ball is on the ground and one or more players from each team who are on their feet close around it. Players must not handle the ball in the ruck and must use their feet to move the ball or drive over it so that it emerges at the team's hindmost foot, at which point it can be picked up. (World Rugby, n.d.)

Sporting Structure: The organizational structure of a sporting entity, including policies, procedures, and by-laws that govern association with or participation under a specific sporting umbrella. In this case, the rugby coaches interviewed all operate within the USA Rugby sporting structure, as coaches, players, and referees are members of the organization and the Division I-A competition structures are facilitated through this organization.

Tackle: When one player wraps their arms around an opposing player, bringing them to the ground. The player is not considered to be tackled unless they have been wrapped in their opponent's arms and at least one knee has touched the ground. 
Touch: When the ball has traveled out-of-bounds in the sport of rugby. This is often referred to as the ball having gone into touch or having found touch.

Touch Judge: One of two linesmen (women) in rugby responsible for monitoring the touch line on either side of the field. Touch judges are also responsible for monitoring kicks at goal.

Turnover: When an employee decides to leave a company and the company subsequently replaces the employee. At times, turnover can be an intentional piece of human resources planning.

Umpire: An official who adjudicates a match. This terminology is often utilized in the sport of baseball.

Verbal abuse: Language intended to cause distress to the target. Examples of verbal abuse may include gossip, profanity, or openly hostile marks regarding one's competency (Brennan, 2001). 


\section{CHAPTER II}

\section{LITERATURE REVIEW}

In Chapter II, a comprehensive review of the extant literature regarding the abuse of sporting officials will be presented. The review examines a myriad of topics relating to referee abuse and is comprised of eight sections: (1) Role of the referee, (2) Referee abuse, (3) Social acceptability of referee abuse, (4) Outcomes of referee abuse, (5) Potential solutions, (6) The role and power of coaches, (7) Conceptual framework, (8) Conclusion. While researchers have completed numerous studies that discuss abuse in the larger social environment, the consideration of abuses within sporting contexts is an emerging field (Brackenridge, 1997b). In recent years, attention has been drawn to the abuses that mentors (coaches, trainers, and managers) have perpetrated against players, focusing primarily on sexual abuse (e.g., Harthill, 2009; Ryba, Schinke, \& Tenenbaum, 2010). As a result, sporting organizations have developed policies aimed at mitigating these abuse behaviors. Policies such as SafeSport, which seek to (1) address all forms of abuse in a sporting context, (2) support athletes of all ages and competitive levels, and (3) provide education and training on both abuse prevention and response (SafeSport, n.d.), have now been adopted by the United States Olympic Committee. Despite the recent push to protect players from abuses within the sporting environment, little attention has 
been paid to the abuses endured by sporting officials. Moving forward, it is important to consider the following:

Reasons for aggression and abuse [against sporting officials], the place of official abuse in cultural practices, situations and strategies that lead to or prevent abuse, the outcomes of abuse for those involved, or the relationship between abuse and decisions by referees to discontinue refereeing. (Simmons, 2006, p. 4)

\section{Role of the Referee}

To explore the concept of referee abuse, the role of the referee within the larger sporting structure will first be considered. Referees, regardless of the sport they officiate, share commonalities in their role as a referee (Dorsch \& Paskevich, 2007). The referee's on-field role is multi-faceted, with responsibilities that include managing the events of a match, applying the rules of the game, maintaining a fair competition, and addressing conflict between sporting participants (Dell et al., 2016). In rugby specifically, the referee's on-field role is defined by the following duties: (1) The referee is the sole judge of fact and of law during a match and must apply fairly all the Laws of the Game in every match; (2) the referee keeps the time; and (3) the referee keeps the score (Law 6.A.4, Rugby Union Laws of the Game). Both rugby officials and referees of other sports seek to fulfill these responsibilities with as little error as possible, yet like the athlete who has missed a pass or struck out, the referee is not infallible (Chiafullo, 1998). Beyond this, the subjectivity required to fulfill the officiating role is an area that is not always understood well.

As Baldwin (2008) explained, every referee may interpret the same rule or law slightly differently. When spectators, players, coaches, and media do not understand the referee's interpretation of laws (rules), the referee may be seen as inconsistent or of low quality (Baldwin, 2008). Based on the sheer number of decisions made by a referee 
throughout the course of a match, mistakes or subjective decisions are inevitable. Thus, it follows that criticism and controversy have become an inherent part of the refereeing role (Dell et al., 2016). The controversy created by the referee role has "been the source of aggravation between officials and players, coaches and spectators and, in doing so, has highlighted the vulnerability of the referee's position" (Cleland, O'Gorman, \& Bond, 2015, p. 552).

Despite the regular criticism of the official, referees are acknowledged as requisite for organized sporting competition. Yet within most sporting organizations, the referee is often relegated to a part-time or volunteer position (Cuskelly, Hoye, \& Auld, 2006). Referees are viewed as providing a service to the game rather than sporting participants in their own right (Phillips \& Fairley, 2014). Phillips and Fairley (2014) suggested that referees cannot and should not be viewed as mere service providers, finding that referees consider officiating a serious leisure pursuit. Although officials themselves may consider officiating to be a serious leisure pursuit, officiating is an endeavor that is rarely portrayed in a positive manner, and vilification of referees is ever-present (Kellett \& Shilbury, 2007). The widely accepted negative social constructions of the role have ensured that "umpires are maligned and afforded little respect" (Kellett \& Shilbury, 2007, p. 211). The connotations associated with refereeing present a setting in which abuse may become an innate consequence of the role (Kellett \& Shilbury, 2007). 


\section{Referee Abuse}

Drawing from the United States Soccer Association, Chiafullo (1998) recognized

referee abuse as follows:

A verbal statement or physical act which implies or threatens physical harm to a referee or a referee's property or equipment. Abuse includes, but is not limited to, the following acts committed [against] a referee: using foul or abusive language toward a referee; spewing any beverage on a referee's personal property; or verbally threatening a referee. (p. 202)

The definition extends those previously put forth (e.g., Folkesson, Nyberg, Archer, \& Norlander, 2002; Friman et al., 2004; Simmons, 2006) where abuse was understood to include verbal and non-verbal behaviors directed at referees that the referee experiences as unpleasant. The first step in eradicating referee abuse is to clarify the problem at hand. A clear and agreed upon definition of referee abuse ensures that all sporting stakeholders are in alignment as to what behaviors constitute referee abuse. Establishing an agreed upon definition of referee abuse may help to promote understanding and buy-in as sport managers seek to eliminate an issue that pre-dates the creation of any of the four major sports leagues in North America.

\section{History of Referee Abuse}

Prior to the 1800 s, most sports maintained that honesty was a construct of the gentile class participating in sport, thus there was no need for a referee (Colwell, 2000). In these settings, it was the responsibility of the team captains to resolve disputes during the competition. However, as sport became more organized in the late 1800 s and the stakes began to rise, the need to ensure a fair competition became paramount. At the same time, the game of rugby was evolving "from a folk game played by ruffians to a recreational activity of custom and ritual for public schoolboys" (Dunning \& Sheard, 2005 , p. 19). Formalization of the game enhanced the requirement for an authority figure 
whose primary task was to ensure fair play. Thus, in 1875, officials were first introduced to the sport of rugby and became a required part of competition in 1885 (Knight, 2009). Like in rugby, referees have become authority figures in nearly all sporting competitions dating back to the 1870s (Yendle, Leverton, Kemp, \& Batts, 2007). The advent of referees as part of sporting competition quickly led to the practice of harassing referees. Slabbert and Ukpere (2010) noted that the referee has been vulnerable to violence for more than a hundred years, and as Colwell (2000) noted, referee abuse has become deeply rooted in the sporting culture since that time.

Despite the longstanding history of referee abuse in sport, little has been done to address the problem. In fact, the 2017 rugby Laws of the Game do not directly reference abuse of the referee but offer 17 examples of foul play against players and the game, and the subsequent consequences are reviewed in depth. For a variety of reasons, these actions against referees within the sporting context have not been explicitly labeled as abuse (Brackenridge, Bringer, \& Bishopp, 2005). Avoiding referee abuse as a concept that should be managed by the Laws of the Game fosters a situation where the recourse for committing referee abuse is variable. At the highest levels of competition, disciplinary procedures address punishments for referee abuse, though the determination of said abuses is done by a disciplinary committee. As a result, the recourse for abuse varies throughout various constituencies. Perhaps resultantly, "no 'line in the sand' is ever drawn. Words no longer suffice-instead, conduct in the form of gesture and intimidation have become more prevalent." (Chiafullo, 1998, p. 206) 


\section{Prevalence of Referee Abuse}

Referees routinely experience dissent and abuse and other forms of aggression at all levels of match. They experience hostile reactions to their decisions and aggressive attempts to influence them from players, coaches, parents, and spectators. Abuse of sport officials is widely understood to be the main reason for a decline in the number of sport officials in Australia (Simmons, 2006). Though physical abuse of officials may receive the most notoriety from the press, Rayner and colleagues (2016) asserted that the predominant form of abuse in rugby union was verbal in nature, accounting for $88.8 \%$ of all reported abuse. The researchers also found that physical abuse accounted for $10.10 \%$ of abuses referees experienced, and mental abuse was the lowest reported form of abuse at $1.27 \%$ (Rayner et al., 2016).

Abuse of sporting officials is not limited to a specific sport. Rainey (1995) found that $11 \%(n=782)$ of baseball and softball officials had been assaulted at least once during their officiating career. Of those assaults, nearly half involved significant physical abuse, including punching, choking, or being hit with equipment. Despite the serious nature of these abuses, most of the occurrences went unpunished. In the basketball context, it was suggested that $13.6 \%(n=721)$ of referees had been assaulted at least once in their careers (Rainey \& Duggan, 1998). Again, nearly half of the perpetrators were not held responsible for their actions, as no punishment was enacted (Rainey \& Duggan, 1998). A subsequent study conducted on behalf of the Australian Sports Commission randomly sampled Australian households and found that referee abuse in non-professional sport is both frequent and increasing in occurrence (Cuskelly et al, 2004). Among study respondents, $77 \%$ reported to be somewhat concerned or very 
concerned about the abuse of officials, and $81 \%$ believed that abuse of officials is never acceptable. Despite this, $16 \%$ of those surveyed believed that referee abuse is acceptable sometimes, and $2 \%$ believed that abuse is always or mostly acceptable (Cuskelly et al., 2004). The acceptance of referee abuse is worth noting, as $18 \%$ of respondents in Australia find no fault with abuse of officials (Cuskelly et al., 2004). In order to quell such perceptions, sporting administrators can no longer turn a blind eye to the problem (Simmons, 2006).

A number of other scholars (e.g., Dell et al., 2016; Rayner et al., 2016; Walters et al., 2016) have supported the notion that referee abuse is ever present. Total Youth Football, a nationally-distributed soccer magazine in the United Kingdom, conducted a survey of referee abuse in the youth game and found that $82 \%(n=574)$ of soccer participants (e.g., parents, players, coaches, spectators, etc.) had witnessed abuse aimed at match officials (Total Youth Football, 2008). Further, the issue of official abuse has become so pervasive that almost all 12 of the soccer referees interviewed by Dell and colleagues (2016) expressed that they had experienced abuse. The notion of systematic referee abuse is further supported by Rayner and colleagues (2016) who found that 67\% $(n=106)$ of English rugby referees had reported being abused during a sporting competition. Further, Walters and colleagues (2016) found that nearly two-thirds of coaches and referees reported that they had witnessed verbal abuse of a referee $(62.7 \%, n$ $=145)$ over the course of the season.

Nearly two decades of scholars' research highlights how widespread referee abuse has become in sport. Referee abuse does not seem to discriminate and appears to be found in sports from softball to rugby, across numerous continents, and from youth to 
professional levels. Despite the widespread nature of referee abuse, the majority of the available research considers the issue from a narrow perspective- that of the referee. Because referees experience abuse from various sources, it is important to create a holistic picture of referee abuses, considering the sources of said abuse and the perceptions of abuse from these perspectives.

\section{Sources of Referee Abuse}

In the sporting context, referees suffer abuses from players, coaches, spectators, and sports media (Baldwin, 2008; Dell et al., 2016; Rayner et al., 2016).

Performance sports in which competition and winning are paramount have dramatically influenced sporting behaviour [sic] in recent years. The value of winning may become such a seductive goal that all thoughts of moral behaviour [sic] are temporarily put aside. (Woods, 2007, p. 170)

As the stakes of competition increase, with youth vying for college scholarships and collegiate players seeking to become professionals, so, too, do the instances of referee abuse in sport. Despite this, the sources of referee abuse have remained relatively consistent since the early 19th century.

Notably, Nazarudin and colleagues (2009) found that $46.8 \%(n=35)$ of rugby referees found verbal misconduct by spectators to be a serious or very serious problem. They also reported that $40 \%$ of officials noted that verbal misconduct by coaches was also a serious or very serious problem in the Malaysian Rugby Union. Beyond the significant concern regarding verbal abuses, physical misconduct by coaches and spectators was also perceived to be a serious or very serious problem, as reported by $25 \%$ $(n=35)$ of Malaysian rugby referees (Nazarudin et al., 2009). Supporting the results of this study, Rayner and colleagues (2016) found that rugby referees between the ages of 18 and 44 attribute most of the abuse they experience to players, while 45 - to 54-year-old 
referees find coaches as the major source of abuse. Referees aged 55 and older cite spectators as being the main perpetrator of abuses. These results support the anecdotes of many sporting participants, showing that referee abuse can come from the field of play, the sidelines, or the stands. Though referee abuse comes from many sources, officials attend more to aggression from players and coaches than aggression from spectators, as they find these sources of aggression more difficult to manage (Folkesson et al., 2002). While spectator abuse is certainly a problem, it appears that the physical distance between the referee and fans mitigates the impact of some of these abuses. Based on these findings, the study of abuses from players and coaches should be prioritized over the abuse behaviors performed by spectators.

In addition to the abuses received from the aforementioned sporting stakeholders, the sporting media can also serve as a propagator of referee abuse. In sport commentary, the referee often becomes the scapegoat for poor team performances. While these reports may be justified at times, the sporting media is also seeking to sell their stories and thus must cater to their audiences accordingly. As much sport reporting has found its home online, from web pages to social media sites, the ability to share information has increased exponentially. As a result, the sporting media has become a promulgator of referee abuse.

\section{Rationale for Referee Abuse}

Previous research has established that referee abuse exists (e.g., Brackenridge et al., 2011; Cleland et al., 2015; Rayner et al., 2016; Simmons, 2006), yet there is a dearth of research that seeks to better understand external perceptions of referee abuse or to ascribe potential solutions. Perhaps the lack of research is a result of the numerous factors 
that contribute to the rationalization of referee abuse. As Cleland and colleagues (2015) suggested:

Part of the explanation may rest in the potential for disparity between those governing the laws of the game [the sport organization], those applying the laws in an ostensibly objective manner (the referees), and those playing the game (the players and their increasing tendency to push the rule boundaries through the desire to win at all costs). (p. 552)

Generally, referees do not attribute abuses to errors on their own part, finding instead that most abuses are "caused by external factors, such as incidents that occurred during the game" (Simmons, 2006, p. 12). Referees' disassociation from responsibility aligns well with ideas presented in the fairness theory. Researchers exploring the fairness theory purport that a central tenet to one's understanding of fairness is the assignment of blame (Greenberg \& Cropanzano, 2001). "When people identify an instance of unfair treatment, they are holding someone accountable for an action (or inaction) that threatens another person's material or psychological well-being” (Greenberg \& Cropanzano, 2001, p. 1). According to fairness theory, those seeking fairness often engage in counterfactual thinking, where they imagine a situation different from reality (Colquitt, Greenberg, \& Zapata-Phelan, 2005), allowing themselves to assign blame to an external party. In instances of dissent or abuse, referees perceive the players' lack of rule knowledge and/or their internal biases as the antecedents rather than considering how their own error may contribute to the frustration of players, coaches, and spectators. Players and coaches simultaneously believe that the referee is erring rather than attributing any negative results to their own behavior or poor performance or preparation. Thus, for players and coaches, abuses are found to result from the perception that a referee's decision is inaccurate or erroneous (Kaissidis-Rodafinos \& Anshel, 2000) or that the referee's decisions are biased. In seeking a scapegoat for their own negative behaviors or 
shortcomings, players, coaches, and spectators alike place blame on the referee in order to protect their personal well-being and promote their perceptions of fairness.

Further, players associate aggression, hesitation, and arrogance with poor refereeing, and thus, these behaviors diminish an individual's perception of fairness (Simmons, 2006). If sporting constituents perceive their match to be unfair, that helps to justify their proclivity toward abusive behavior. Additionally, "players' concern for referees to be competent and dependable parallel organizational justice findings that employees perceive unfairness when there are deviations from expected procedure" (Van den Bos, Vermunt, \& Wilke, 1997, p. 96). The incongruence of perceived realities among referees, coaches, and players highlights the importance of considering referee abuse from perspectives beyond that of the referee.

Additionally, the referee must consider the behaviors and attitudes that they present to players, ensuring that they maintain their composure, even in moments of frenzy within a competition. A referee's interaction with players and coaches can be incredibly important to managing a sporting competition; as such, referees often employ deliberate strategies to control external perceptions. Clear communication, sensitivity, and proactivity are professed to enhance the acceptance of an official's decision (Simmons, 2006). These suggestions necessitate further research regarding referee abuse from an external perspective. Better understanding of how coaches and players perceive situations that lead to abuse will allow for robust strategies to counteract these occurrences. Because a unified approach to the problem of referee abuse has not been taken, referees are forced to employ several individualized strategies to cope with the stress and abuse they face within their profession. 


\section{Coping with Referee Abuse}

As previously mentioned, referees have identified a pattern of abuse; thus, coping skills have become an important part of their training as they learn to contend with this issue. Stressful events, including referee abuse, are shown to trigger a referee's coping response. Coping is defined as the psychological and behavioral efforts to master, reduce, or tolerate demands (Folkman \& Lazarus, 1985), and in this case, those efforts required by the refereeing profession. To remain in a high-stress position such as officiating, one must be able to cope with the stressors that exist. As previously discussed, officials are expected to manage numerous stressors during every match (e.g., abuses from players, coaches, spectators, referees; presence of media; potential for injuries; responsibility for the outcome of the match; working with a partner or team; etc.). Both the type of stressor and cultural factors have been shown to impact the referee's use of behavioral, emotional, and avoidance coping strategies during a match (Anshel \& Weinberg, 1996). Such strategies may include changing one's communication style, concentrating harder, and ignoring unbecoming behaviors. Anecdotally, coping strategies seem to be successful in alleviating stress when implemented, but little work has been done to quantify their value for referees.

One specific coping strategy that has been investigated in greater depth is that of reframing referee abuse. Kellett and Shilbury (2007) indicated that although abuse does exist for referees, those who have been retained as officials are able to use the reframing of abuse as a coping strategy. By doing so, the abuse becomes less personal and, instead, a product of their external environment. Reframing allows veteran referees to adapt to their surroundings rather than being bogged down by the abuse itself. Novice referees, 
however, have been shown to experience higher levels of stress (Anshel \& Weinberg, 1996). With inadequate training and development programs, these referees may never reach a veteran status where they have learned to reframe abuse. Further, as reframing abuse becomes an acceptable part of the veteran referee's experience, we must consider the responsibility placed on the referee to persevere despite a hostile work environment.

\section{Social Acceptability of Referee Abuse}

Socially acceptable behaviors are understood as those that do not violate social norms, regulations, or laws. Within the sporting world, abuse of officials is generally considered socially acceptable (Kellett \& Shilbury, 2007). Even referees have come to expect and accept abuses (Kellett \& Shilbury, 2007), expressing that often, retained referees are able to reframe abuses. The president of the National Association for Sport Officials in the United States issued a message during a recent video press release that insinuated referee abuse was an issue for which a resolution should not be expected:

First, I think we need to agree that we will be unable to change the expectations of those who use our services, we need to accept what they expect, it ain't fair, it ain't especially reasonable, and it ain't gonna [sic] change. Let's get over it. Suggestions - toughen up, keep our heads down with our antennas up, we need to better understand when to talk and when to shut up. Relying on others to make our world better is hope, but it isn't a strategy. (Mano, 2017, 1:58)

Such statements do little to combat the social acceptability of referee abuse but rather normalize the behavior. Normalization is a social process through which a behavior becomes incorporated into the everyday world, thus falling within the scope of expected behavior for a specific group (May et al., 2009). It has been suggested that sport can foster undesirable behaviors through its subcultural norms (Nelson, 1980). "In this [way], each sport has allowed certain actions, such as yelling at the umpire or arguing with the referee, to become traditional conduct which is tolerated and even expected" (Nelson, 
1980, p. 2). Such normalized sporting behavior has been termed a tradition of deviance (Loy, McPherson, \& Kenyon, 1978). A tradition of deviance is a product of a cohesive set of norms that favors deviant behavior. For example, highly identified fans have been found to "support yelling at or heckling a player, referee, or coach" (Rudd, 2016, p. 181). In this case, the deviant behavior of abuse becomes acceptable because it exists in a sporting context where referees are not viewed as human. Instead, referees are seen as outsiders responsible for organized and fair play within a sporting event (Chiafullo, 1998). Without a personal tie to said referee, dehumanization occurs. As a result, "verbal abuse and physical confrontation is no longer seen to affect a person, but rather, an entity. Decorum is cast aside and impulse reigns" (Chiafullo, 1998, p. 214). There are few other contexts where such abuses would be permitted, yet in the sporting environment, referee abuse is allowed and at times applauded. If we consider the same situations of abuse in another context, many would quickly come to the defense of the victim.

Like victims of abuse in other contexts, referees seem to rationalize abuse, at times minimizing the problem. As Kellett and Shilbury (2007) found:

Although umpires were cognizant of the fact that the abuse they receive is contextually specific, and unacceptable according to "normal" rules of social interaction and engagement, they did accept it as part of their role. Moreover, it was evident that "normalizing" abuse in this context was reinforced by the system. (p. 220)

The referees interviewed in Kellett and Shilbury's (2007) study utilized nearly identical terminology to describe the ways in which they responded to abuse. The identical descriptions of abuse denote a "learned rather than natural response" (Kellett \& Shilbury, 2007, p. 222) to referee abuse. In fact, it is often suggested that experienced referees have been taught to avoid taking abuse personally, as abuse may be beyond the control of the referee (Folkesson et al., 2002; Simmons, 2006). While disassociation from abuses may 
be required in order to continue operating in a hostile environment, ignoring referee abuse may further normalize the issue.

Walters and colleagues (2016) found that 19.2\% $(n=55)$ of coaches and referees purported to have never witnessed inappropriate behaviors in youth sport. Based on the prevalence of abuses in sport, this finding suggests that these inappropriate behaviors are being overlooked or are considered acceptable within the sporting context. One such participant in this study was able to identify referee abuse behaviors as inappropriate but found them to be acceptable if the referee were making decisions that did not support the success of their team (Walters et al., 2016). As a result, these behaviors may have become so normalized that some sporting participants could even view them as acceptable.

Rudd (2016) supported the notion that sport participants find some referee abuses to be warranted. Participants in this study indicated that though it was "morally wrong to throw objects or cast vulgar obscenities, it is part of the game to heckle opponents or yell in disgust with a referee's officiating" (Rudd, 2016, p. 182). The discrepancy between participants' perspectives of abuse may result from a lack of understanding of sportsmanship or an unclear understanding of abuse. If participants do not understand what constitutes abuse behaviors, they are also less likely to report the behaviors (Rayner et al., 2016). Walters and colleagues (2016) found an interesting difference in reporting behaviors between referees and coaches, with referees reporting more inappropriate behaviors than coaches. Coaches may report a smaller number of abuse behaviors, as they may result from the in-game behaviors of coaches themselves. Coaches have been found to contribute a "high volume of negative comments" (Walters et al., 2016, p. 68) 
during sporting events. Thus, it is reasonable to believe that because coaches are regularly participating in the verbal abuse of referees, they may be unaware or more tolerant of language that others would typically view as abusive. The lack of awareness or general acceptance of referee abuse spreads beyond the on-field participants in sport. Often, the public mirrors the behaviors of coaches, discounting sports-related violence and abuses as part of the game, but such an attitude is ultimately problematic as abuse does have an impact on its victims (Fields et al., 2007). To date, little work has considered a coach's understanding of referee abuse or the antecedents that prompt one to partake in referee abuse. As such, this study will seek to fill the gap in the literature revealing coaches' perceptions of referee abuse and illuminating potential causes and solutions.

\section{Outcomes of Referee Abuse}

Numerous negative outcomes have been found to result from the experience of referee abuse. For individual referees, abuse can impact their mental health, leading to performance issues, stress, and ultimately burnout or dropout (Goldsmith \& Williams, 1992; Taylor, Daniel, Leith, \& Burke, 1990; Voight, 2009). The impact of abuse also extends beyond the experiences of a single referee. Referee abuse may also impact the fairness and safety of a match along with the quality and availability of referees to oversee sporting contests.

\section{Negative Outcomes Related to Stress}

Officiating is often considered a highly stressful occupation (Baldwin, 2008). In fact, it has been suggested that the scope of referees' duties makes them among the most stressed individuals in a sporting contest (Anshel \& Weinberg, 1995; Rainey \& Hardy, 
1999; Rainey \& Winterich, 1995). Zoller (1985) noted that the job of sports officiating is so stressful that it ranks among air traffic controllers, inner city teachers, and police officers in terms of levels of occupational stress. Because of its high stress nature, officiating has been referred to as the worst job in sport (Baldwin, 2008).

Stress in the officiating role often manifests due to the perception that the official has inaccurately applied a rule, often for the benefit of the other team (Anshel et al., 2013). Making the wrong call or making an error can result in the referee receiving abuse from coaches, athletes, and/or spectators (Rainey, 1995), which often increases stress. In 1995, Rainey found that officials experience four categories of acute stress, including (1) fear of failure (e.g., errors in decision-making), (2) fear of physical harm (e.g., assaults by sporting stakeholders), (3) time pressure (e.g., scheduling, external constraints), and (4) interpersonal conflict (e.g., managing abusive participants). Anshel and Weinberg (1995) found that making a "wrong" call, verbal abuse by coaches, threats of physical abuse, and being in the "wrong" position when making a call were the most intense sources of stress for sporting officials.

Various combinations of the aforementioned stressors have been found in many subsequent studies, with the fear of physical harm (e.g., assault by a player/coach/ spectator) and the fear of failure (e.g., making an incorrect decision) serving as two stress-inducing factors that have remained consistent in almost all studies of referee stress (e.g., Goldsmith \& Williams, 1992; Kaissidis-Rodafinos, Anshel, \& Porter, 1997; Rainey, 1995; Stewart \& Ellery, 1998; Voight, 2009). More recently, verbal abuse by coaches has been ranked highest among the sources of stress for sporting officials (Walters et al., 2016). Consequently, Dorsch and Paskevich (2007) found the threat of physical abuse to 
be greater than any other stress factors, including verbal abuse. While the greatest sources of stress may vary by referee, the consistencies of these findings confirm that officiating is a high-stress vocation and that abuse — physical or verbal — contributes to the stress felt by officials.

Anshel and Weinberg (1995) highlighted how exposure to a violent environment can lead to symptoms associated with clinical stress. Thus, referees' continual exposure to stress is concerning for both their physiological and psychological well-being. Physiologically, stress can result in hypertension; over time, that can result in long-term increased blood pressure. Stress also increases the risk of ulcers and may suppress the immune system, exposing individuals to increased risk of a variety of infectious diseases (Sapolsky, 2000).

Psychologically, individuals may come to a point where they no longer try to cope with the stress that they are experiencing, also known as depression. The relationship between referee abuse and negative outcomes as a result of stress highlights the importance of understanding referee abuse with the hope of decreasing its presence in sport. In addition to the effects of stress on the individual referee, referee abuse may also hamper the referee's ability to provide a fair and safe sporting competition.

\section{Fair and Safe Sporting Competitions}

Experiencing aggression and abuse within the sporting context has been shown to impact a "referee's concentration, performance, motivation, and [has led to them] doubting their own decision-making” (Friman et al., 2004, p. 653). These individual responses highlight the importance of curbing referee abuse, not only to ensure the personal well-being of the referee but also to ensure the quality of a sporting competition. 
Dell et al. (2016) found that a number of referees felt underprepared for the abuses that they were experiencing, and this impacted their self-confidence and created a sense of isolation and vulnerability.

When referees experience abuses and thus feel vulnerable, they have a tendency to react with anger and seek retribution (Lazarus, 1991, 1993). Referees seeking to rebound from abuses tend to favor the opposition in their decision-making. Ackery and colleagues (2012) found that $92 \%(n=632)$ of referees acknowledged experiencing anger and abuse directed personally toward them; of those referees, $32 \%$ reported that these behaviors had influenced the outcome of the game. A referee's response to referee abuse has the potential to create an unfair bias as the referee victimizes the offending player's or coach's team. Referee abuse thus has the potential to impact the fairness of a sporting contest.

Beyond the consideration of fairness, referees are also tasked with the maintenance of a safe sporting environment through the promotion of safe play and rule enforcement (Collins, Fields, \& Comstock, 2008). The goal of maintaining a safe sporting environment can often be derailed as a result of referee abuse. Referees report that verbal or physical abuse of referees by coaches, fans, and parents has led to an increased risk of injury for participants. As mentioned earlier, referee abuse can lead to distracted referees; thus, a referee's ability to oversee a safety in a sporting competition becomes impaired. Further, the referees interviewed by Ackery and colleagues (2012) indicated that abuse behaviors resulted in the referee losing control of the match more than 50\% $(n=632)$ of the time. Losing control of the match implies that the referee no longer has the ability to control player or coach behaviors, thus putting participants at an 
increased risk for injury. Not only does referee abuse have the potential to cause stress and impair the quality of the sporting competition, but referee abuse may also lead to referee discontinuation and dropout.

\section{Referee Recruitment and Retention}

As demonstrated in other fields, continued abuse is likely to decrease referees' satisfaction with their vocations and increase the potential for discontinuation (Rowe \& Sherlock, 2005). As referees discontinue participation as a result of abuses (Anshel \& Weinberg, 1995; Dell et al., 2016; Rainey, 1995), sporting organizations face a problem: referee retention. Retention is considered the preservation of an official from one season to another. As such, a retention rate can be produced by dividing the number of officials who have continued into the next season by the number of available officials from the close of the previous season (Cuskelly et al., 2004). When considering retention, the goal is to increase the longevity of officials and reduce the average attrition rate (Warner et al., 2013). Attrition, or lack of retention, has been shown to result in high replacement costs resulting from lost productivity, retraining costs, and administrative costs (Al-Emadi, Schwabenland, \& Wei, 2015). Because of the high cost of replacing referees, sport managers should prioritize the development of practices that support referee retention when seeking to eliminate the officiating shortage (Kim, 2017).

Scholars have discovered dismal referee retention rates (e.g., Brackenridge et al., 2011; Cuskelly et al., 2004; Oke, 2002; Sabaini, 2001). Cuskelly and colleagues (2004) found that some sport associations in Australia reported a first-year attrition rate of up to $60 \%$. Further, a study from the National Association of Sports Officials (NASO) regarding referees in the United States found that at least one-third of referees quit after 
their first year (Sabaini, 2001). In Australia, New South Wales has had to replace nearly $25 \%$ of its soccer referees annually due to dropout (Oke, 2002). In England, the English Football Association (FA) reported that poor behavior, including referee abuse, resulted in the loss of $17 \%$ (nearly 5,500) of referees in the one-year span from the 2007-2008 to the 2008-2009 season (Brackenridge et al., 2011). Systematically, referee dropout as a result of sustained referee abuse is a serious problem for the future of sport, as the loss of referees is often exceeding those being replaced (Dell et al., 2016). Without enough referees, sporting organizations are forced to decrease the availability of competitions or utilize obscure scheduling to meet participant demand (Ridinger, 2015).

When referees drop out as a result of abuses, the requisite for referee recruitment has become an unexpected outcome. Referee recruitment is defined as successfully placing a new referee into the officiating role (Cuskelly et al., 2004). Recruitment does not consider the longevity of the referee but rather seeks to increase the quantity of officials at the outset, providing an ample number to meet demand. While recruitment could be a valuable tool to increase the number of active referees, the selling of a positive image of sports officiating can also be difficult. In most instances, the media portray officials in a negative light, focusing on abuses or performance errors rather than emphasizing efficiencies and successes (Cuskelly et al., 2004). Beyond this, players and fans alike see referees abused at matches on a regular basis. As such, the impact of referee abuse is twofold: current referees are discontinuing participation, and potential referees may be overlooking officiating as a viable pursuit. Referee abuse negatively impacts the individual referee and the collective sporting community; exploring referee 
abuse thus becomes important for the promotion of individual well-being and the sustenance of organized sporting competition.

\section{Potential Solutions}

To diminish the impact of referee abuse for the individual and sporting organization, sport managers must take a holistic approach. Solutions cannot be created and implemented by the referee alone. Rather, sport managers must engage with external sporting stakeholders, including coaches and players, to develop organizational strategies that will influence new and continued participation in officiating. While many negative outcomes of referee abuse have been made apparent, it is time that potential solutions are considered. Thus far, legislation, public information campaigns, punitive measures, and training have been suggested to help quell referee abuse in sport.

\section{Legislation}

Over the last 40 years, sporting organizations have enacted a number of policies seeking to curb referee abuse. Perhaps the most significant referee abuse policy has come in the form of new laws. In 1987, the National Association of Sport Officials (NASO) created model legislation that would make attacks on officials a criminal offense. The model legislation outlines the following:

Section 1: Any person who physically assaults any sports official at any level of competition, within the confines or immediate vicinity of the athletic facility at which the athletic contest in which the sports official was an active participant, shall be guilty of a crime (misdemeanor, felony, etc.) which shall be punishable by a fine of $\$ 10,000$ and/or imprisonment to a maximum of three (3) years.

Section 2: Sports officials are defined as those individuals who serve as referees, umpires, linesman (sic), and those who serve in similar capacities but may be known by other titles and are duly registered or members of a local, state, regional or national organization which is engaged in part in providing education and training to sports officials.

Section 3: The law shall take effect immediately. (Chiafullo, 1998, p. 219) 
Stemming from this proposed legislation, 23 of the 50 U.S. states have enacted laws that seek to restrain the instances of abuse against sport officials. These laws call for stiffer sentences for those convicted of assaulting or threatening sport officials (Chiafullo, 1998). Legislation has been mentioned as a viable deterrent to referee abuse (Chiafullo, 1998), though to date, little research is available to validate the impact of these new laws.

\section{Public Information Campaigns}

Beyond adopting legislation, sporting organizations have sought to enact programming aimed at deterring poor behavior against match officials. A common method employed by sport organizations is that of a public information campaign. The success of such a campaign is dependent on its ability to capture the attention of the appropriate audience and present a clear message that has the ability to influence beliefs to set the stage for the creation of desired social outcomes (Weiss \& Tschirhart, 1994). Public information campaigns "are generally passive instruments in that they intend to make a large targeted population more knowledgeable of a particular issue at a relatively low cost to inform choices and alter behavior" (Cleland et al., 2015, p. 554). While public information campaigns often succeed in increasing awareness and can impact the attitudes of the target market, this does not necessarily translate into improved behavior. The difficulty of changing entrenched behaviors has been cited in physical activity campaigns aimed at modifying health-related social norms (Randolph \& Viswanath, 2004). Despite the difficulty of affecting behavioral change through public information campaigns, the English Football Association (FA) attempted to curb referee abuse through a campaign called Respect. Cleland and colleagues (2015) found the following:

Whilst the capacity for the FA to act on poor behavior to match officials would appear to be significant in terms of knowledge, expertise and resources available 
to the governing body, the sheer number of participants, coaches and officials participating in the grassroots game presents challenges for the FA to implement change or tackle deeply embedded cultural norms that expose referees to criticism and abuse. (p. 554)

\section{Data Tracking and Reporting}

Data tracking and reporting is incredibly important in the development of these public information campaigns and laws. Data collection and the subsequent analysis of data ensure that policies and best practices are derived from statistical information rather than hearsay. The systematic reporting of data is critically important in the creation of any program or policy, but the data must be accurate to be effective. As such, all sporting stakeholders must take an active role in reporting instances of referee abuse. It is from such data that the assumptions of victims can be tested, appropriate punitive measures may be taken, and subsequent policy interventions can be planned (Brackenridge et al., 2005). Beyond the accuracy of recorded data, appropriate reporting procedures must also be in place. These procedures must ensure the safety of the reporter to eliminate future repercussions and eliminate fear of reporting. These procedural measures certainly have their place in eliminating referee abuse, but it is the buy-in of sporting stakeholders that will truly allow any program or policy to succeed.

\section{Punitive Measures}

Another potential solution that has been put forth is the referee's ability to enact punitive measures against the offending sport participant. Such measures include removing a participant or a coach from the field of play. It seems, however, that when referee abuse occurs at a level that warrants a send-off, referees inconsistently utilize these punitive measures. At higher levels of competition, cameras can detect such abusive language and action; however, consider the following: 
Inconsistency in applying such policy tools not only undermines a "top-down" approach towards behavior change but also appears to present mixed messages to footballers and stakeholders at the grassroots level. For those referees at the grassroots level, this then has an impact on their ability to control a Saturday or Sunday match. (Cleland et al., 2015, p. 557)

Beyond these on-field measures, sporting organizations also have the ability to enact punitive measures such as competition bans. Disciplinary committees, when they exist, can hear cases related to referee abuse and determine the appropriate punishment for the offending participant. The intended outcome of such punishments is the prevention of repeat behavior and the deterrent of similar behavior from other sporting participants.

\section{Training}

Beyond punitive measures, training has been suggested as one way to both decrease abuse behaviors and prepare referees to manage such situations. For nonreferees, sensitivity training that addresses information, such as what constitutes referee abuse, the policies that the sporting organization has adopted regarding referee abuse, and how to be a referee ally, may serve as a starting point to promote understanding around the issue. For referees, it has been noted that education should be improved to help them better identify abuse and cope with such situations. Simmons (2006) explained:

[All referees should] be taught very explicitly: that a decision made by a neutral, competent and well-positioned referee is likely to be more correct than an observation made by people with an interest in one of the teams. . . . [They should also learn] techniques for depersonalizing abuse, critics to expect to encounter, and how to recognize, player, coach and spectator behaviors that create uncertainty in the referee's mind, or somehow deter the referee from awarding against their team by making the consequences unpleasant. [Finally, it is important to reiterate] that they will make mistakes, and it's important to learn from them. (p. 13)

In these ways, it has been suggested that both referees and non-referees can be trained to help eliminate referee abuse. 


\section{The Role and Power of the Sport Coach}

The role of the sport coach extends beyond teaching athletes how to execute sport skills. In fact, within the sporting context, a coach is often considered to be not only a teacher of sport skills but also a mentor (Bloom, Durand-Bush, Schinke, \& Samela, 1998). By definition, it is the mentor's role to serve as an "adviser, coach, motivator, facilitator, and role model within a contextual setting" (Kerka, 1998, p. 4). By serving as mentors, coaches teach athletes life skills that translate beyond the playing field and often remain engrained throughout their lives (Walton, 1992). In the sporting arena, coaches have been found to be the most frequent mentor of athletes (Perna, Zaichkowsky, \& Bocknek, 1996) and have been shown to be particularly important to an athlete's development during college (Martens, Dams-O'Connor, \& Beck, 2006). Upon entering college, athletes are freed from their parental oversight for the first time. It is at this time that student athletes are able to assert their own independence and reevaluate their beliefs, values, and future goals. Despite the newfound autonomy of college student athletes, they are not unbound from the influence of their peer group or mentors. At such a transitional period in one's life, "coaches can exert a considerable influence" (Mastroleo, Marzell, Turrisi, \& Borsari, 2012, p. 64). In fact, Martens and colleagues (2006) found that a collegiate coach's influence may be greater than any other individual on campus.

Wielding such great influence, coaches have the ability to impact a large portion of the collegiate sporting culture and environment. Consequently, coaches' influence makes them unique candidates for study, as their perceptions and expectations of referees may impact sport more broadly than other sporting stakeholders. 
Coaches at the collegiate level have been shown to influence athletes' uptake of dietary supplements (e.g., Scofield \& Unruh, 2006), the use of performance-enhancing substances (e.g., Ranby et al., 2009), and drinking behaviors (e.g., Seitz, Wyrick, Rulison, Strack, \& Fearnow-Kenney, 2014). These studies have suggested that coaches are able to influence the behavior of others through the Social Norms Theory based upon their expectations and the behaviors that they will accept (Hahn-Smith \& Springer, 2005). The Social Norms Theory has also been used to show that individuals inflate their perceptions of the approval of their problem behaviors (Borsari \& Carey, 2003). As such, individuals may increase their own problem behavior in order to align with their inaccurate view of normal behavior and their incorrect perceptions of approval. This theory has been applied to collegiate athletes and their coaches in regard to drinking behaviors, as athletes have been found to drink more if they believe that their coaches approve of their drinking behaviors (Seitz et al., 2014). The increase in negative behaviors, if approved by a coach, is likely to hold true for other negative behaviors, including referee abuse. If a coach is perceived to approve of referee abuse, it is probable that players will continue to partake in such behaviors or even increase the amount of referee abuse in which they participate.

As previously mentioned, players, coaches, spectators, and the media are all guilty of referee abuse. But of these groups, coaches are thought to be the most influential in the sporting environment, especially in youth sport. Thus, this study will consider coaches' perceptions of referee abuse as a coach can maintain a complex combination of many roles, including that of an adult-role model, possible surrogate parent, potential peer, and possible prevention agent (Kirschenbaum \& Smith, 1983). 
In fact, the coach has become so influential that a 1996 study in Canada found that $96 \%$ of children surveyed expressed that their coach was more influential in their lives than their parents or teachers (LeBlanc \& Dickson, 1997). With such a broad influence, coaches have a responsibility to expose their players to positive sporting behaviors, but instead we often see coaches "teaching their teams to infringe" (Rayner et al., 2016, p. 75) or modeling inappropriate behaviors, such as the harassment or abuse of referees. Despite being some of the worst perpetrators of referee abuse, coaches have the ability to incite a shift in the cultural norms of sport. Coaches are seen as leaders and role models (Raakman et al., 2010) and are often followed blindly by their players. Should coaches decide to take a stand against referee abuse, their players would likely follow suit. Coaches standing against referee abuse would be a positive step forward in limiting on-field referee abuse and would serve as a unified statement to spectators that referee abuse is not welcome. While a unified approach against referee abuse would require a departure from deeply engrained cultural norms, it may be the catalyst that drives an increase in referee numbers and allows organized sport to continue for future generations.

\section{Conceptual Framework}

A conceptual framework is a visual model that explains the main features of a study, including key factors, concepts, or variables and any potential relationships among them (Miles \& Huberman, 1994). In the case of a phenomenological study, it is important to note that this framework serves as a guide to the phenomenon being studied but must often change in response to what is learned from the participants (Strauss, 1987). As Liehr and Smith (1999) described, a framework for research should provide guidance for the researcher in developing relevant research questions and selecting an appropriate 
methodology. The methodological assumption was that throughout the study, emerging framework design, shaped by the researcher's experience, along with inductive logic and data analysis, was utilized (Creswell, 2013). As such, qualitative inquiry must take account of theories and perspectives of those studied in order to determine how a conceptual framework fits or should be modified in order to appropriately portray the perspectives of the participants. Dressman (2009) noted that researchers often present their results in a way that completely supports their conceptual theories. A lack of critical analysis undermines the credibility of the findings and can also blur the outcomes of the study and our ultimate understanding of a phenomenon. Therefore, as data were collected, the researcher determined whether the findings aligned with the framework, analyzed discrepancies, and questioned whether or not the framework could be used to explain them. Within a phenomenological study, the researcher did not utilize hypothesis testing but rather allowed the participants' data to inform the outcomes of the study and any subsequent alignment with existing theory (Hycner, 1985).

In order to consider rugby referee abuse through the lens of rugby coaches, an ecological-transactional framework served as a tentative model. An ecologicaltransactional framework stems from the works of Bronfenbrenner (1979), Belsky (1980), and Cicchetti and Lynch (1993). Bronfenbrenner (1979) proposed the Ecological Systems Theory of human development. According to Bronfenbrenner (1979), five environmental systems are said to impact an individual's development, with the microsystem being most impactful. In the Ecological Systems model, the microsystem referred to the institutions that directly impacted the child's development, including church and family. The next environmental system, the meso system, interconnected the relationships between the 
microsystems; for example, the relationship between family members and teachers. The third level of this model, the exosystem, linked the social setting beyond an individual's immediate context. An example of this system may be a parent whose work schedule has changed. While there was no direct impact on the child, there may be a knock-on effect as the parent is unavailable during a child's waking hours. The macrosystem goes on to describe the culture in which a child exists. Finally, the chronosytem takes into account the timing of activities. This level seeks to consider the impact of sociohistorical circumstances on a child. Within each system exist rules and norms that ultimately impact the child's development. The seminal work of Bronfenbrenner (1979) has impacted the way in which a child's development is considered throughout his or her life's course.

An extension of theory derived from the Ecological Systems theory was proposed by Belsky (1980) in order to provide a framework for understanding the broader environment, or ecology, in which child maltreatment was occurring. This ecological model included the following:

Four levels of analysis: (1) ontogenic development, which includes factors within the individual that are associated with being a perpetrator of child maltreatment; (2) the microsystem, which includes factors within the family that contribute to the occurrence of child maltreatment; (3) the exosystem, which includes aspects of the communities in which families live that contribute to child maltreatment; and (4) the macrosystem, which includes the beliefs and values of the culture that contribute to the perpetuation of child maltreatment (Belsky, 1980).

A slight derivation created a model to explore abuse beyond the perspective of the individual, considering instead the entirety of one's environment. It is from Belsky's (1980) model that Cicchetti and Lynch (1993) proposed the ecological-transactional model of vulnerability (See Figure 1). Noting that the variables encompassed by the four levels of analysis were likely to interact with one another, the transactional piece was 
added to the model. The transactional element of the model presupposes that each level of one's ecological environment may be impacted by other levels. For example, familial factors (within the microsystem) are likely to be influenced by the exosystem level (the community). In this way, family values or perceptions of right and wrong may be shaped by the community in which they live. Thus, the potential for a transactional component between each of these levels was illuminated in Cicchetti and Lynch's (1993) model. 


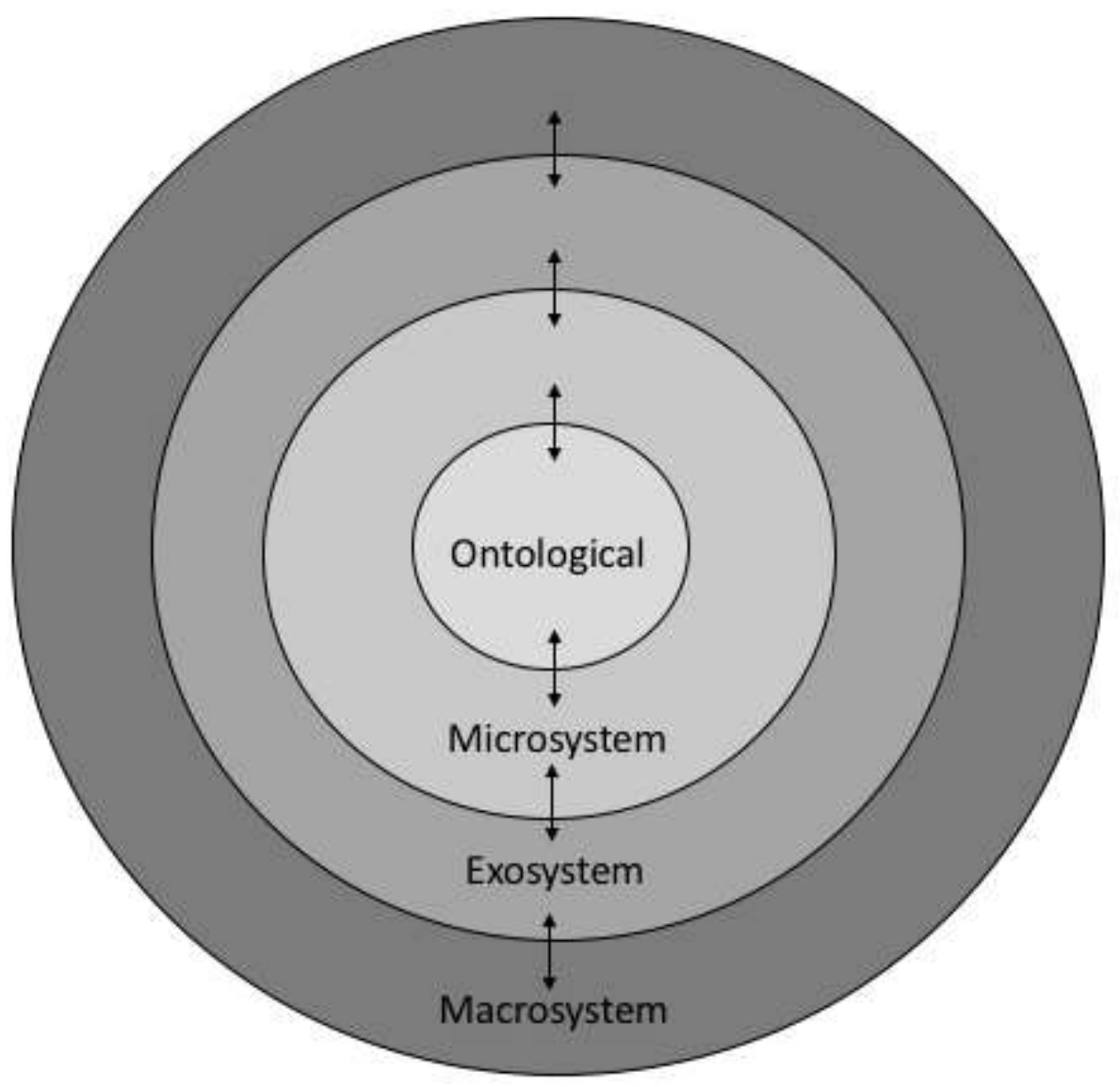

Figure 1. Cicchetti \& Lynch's (1993) Ecological Transactional Model. 
The ecological-transactional framework has the potential for widespread application and has previously been utilized to assess topics closely related to referee abuse, including community violence (e.g., Cicchetti \& Lynch, 1993) and emotional abuse in the coach-athlete relationship (e.g., Stirling \& Kerr, 2014). In both examples, the ecological framework looked at vulnerabilities, and variables of risk were organized by four categories: (1) ontological development, (2) the microsystem, (3) the exosystem, and (4) the macrosystem. These transactional categories describe factors that may contribute to negative behaviors (e.g., violence or emotional abuse) and how these variables may interact (Stirling \& Kerr, 2014). Stirling and Kerr's (2014) model of abuse in the coachathlete relationship (Figure 2) depicts such variables in their respective ecological level. 


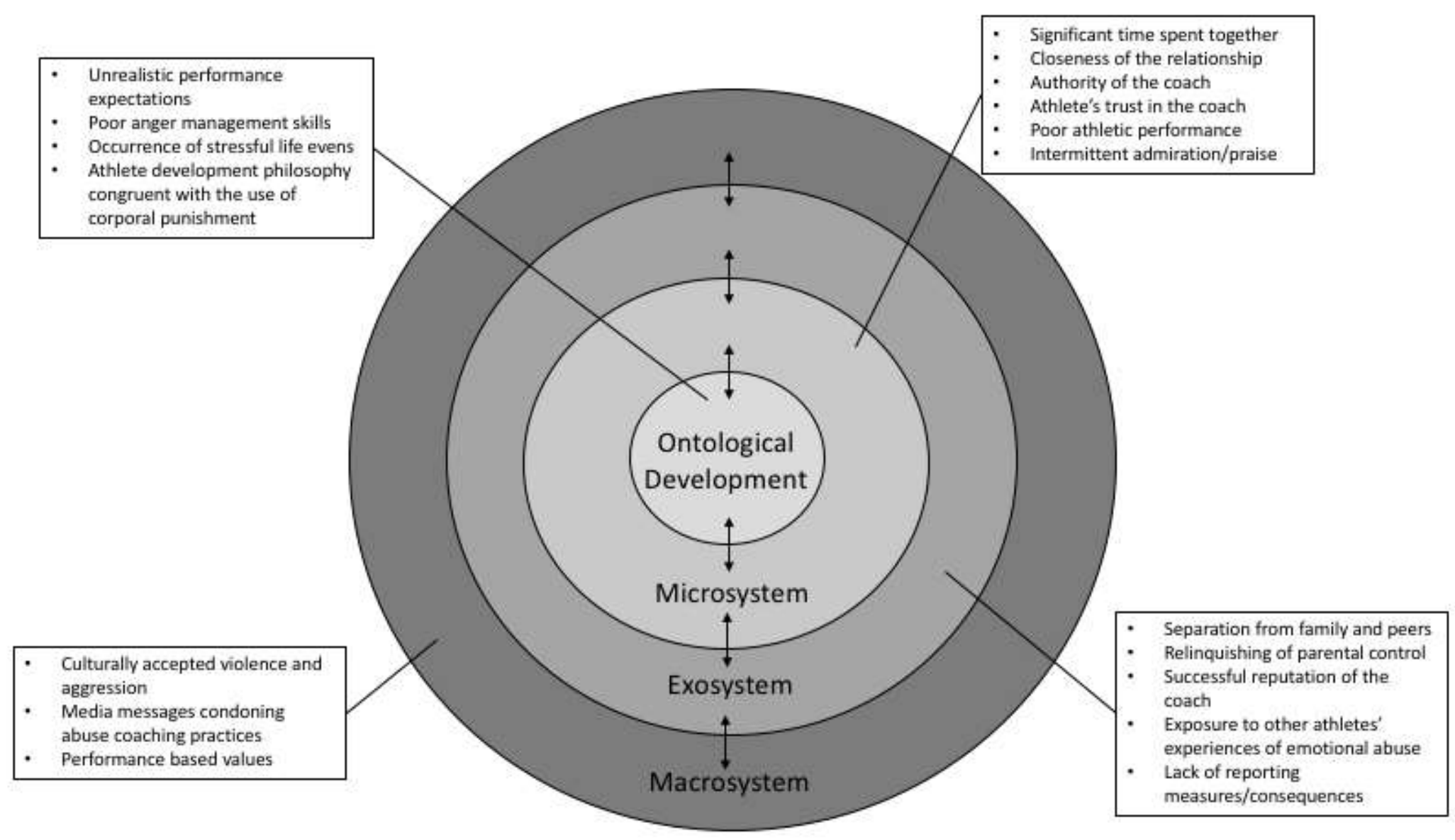

Figure 2. Stirling \& Kerr's (2014) model of abuse in the coach/athlete relationship. 
Stemming from Bronfenbrenner's (1979) early work in child development, the ecological-transactional framework can be applied to nearly any phenomena that may be influenced by ecological factors. As Stirling and Kerr (2014) have shown, the framework assists in the organization of factors that impact emotional abuse in the coach-athlete relationship. In its previous uses, this model has suggested that there are a number of factors that may contribute to negative behaviors and has subsequently organized these factors into four categories. Similarly, this framework was applied to this research project, exploring factors that impact referee abuse perceptions and behaviors within the rugby coaching population.

The ecological transactional framework helped to frame the research questions put forth in this study. As such, this phenomenological study was guided by an overarching question seeking to explore the phenomenon of referee abuse from the perspective of coaches but sought also to understand the factors that underlie such perceptions or behaviors. Clear guidance was taken from the ecological-transactional framework in developing this second research question. Based on previous research using this model, it became clear that numerous environmental factors have the potential to impact one's understanding or action related to a specific topic. Thus, similar factors may impact a coach's understanding of referee abuse or his or her own referee abuse behaviors. Though four levels of ecological analysis have been outlined in Cicchetti and Lynch's (1993) model (and utilized by Stirling \& Kerr, 2014), a phenomenological study should not be bound by existing theory. As a result, specific interview questions and follow-up questions have been derived from the notion that various environmental factors 
may impact a coach's perceptions or tendencies toward abuse, but the researcher did not organize the exploration of factors based solely upon the four ecological levels outlined by this model. Stemming from the work of Cicchetti and Lynch (1993), the interview questions sought to explore the overall ecology in which referee abuse exists. As such, participants' responses were derived directly from their own experiences with referee abuse and may have expanded beyond the confines of the four levels of ecological analysis previously put forth. 


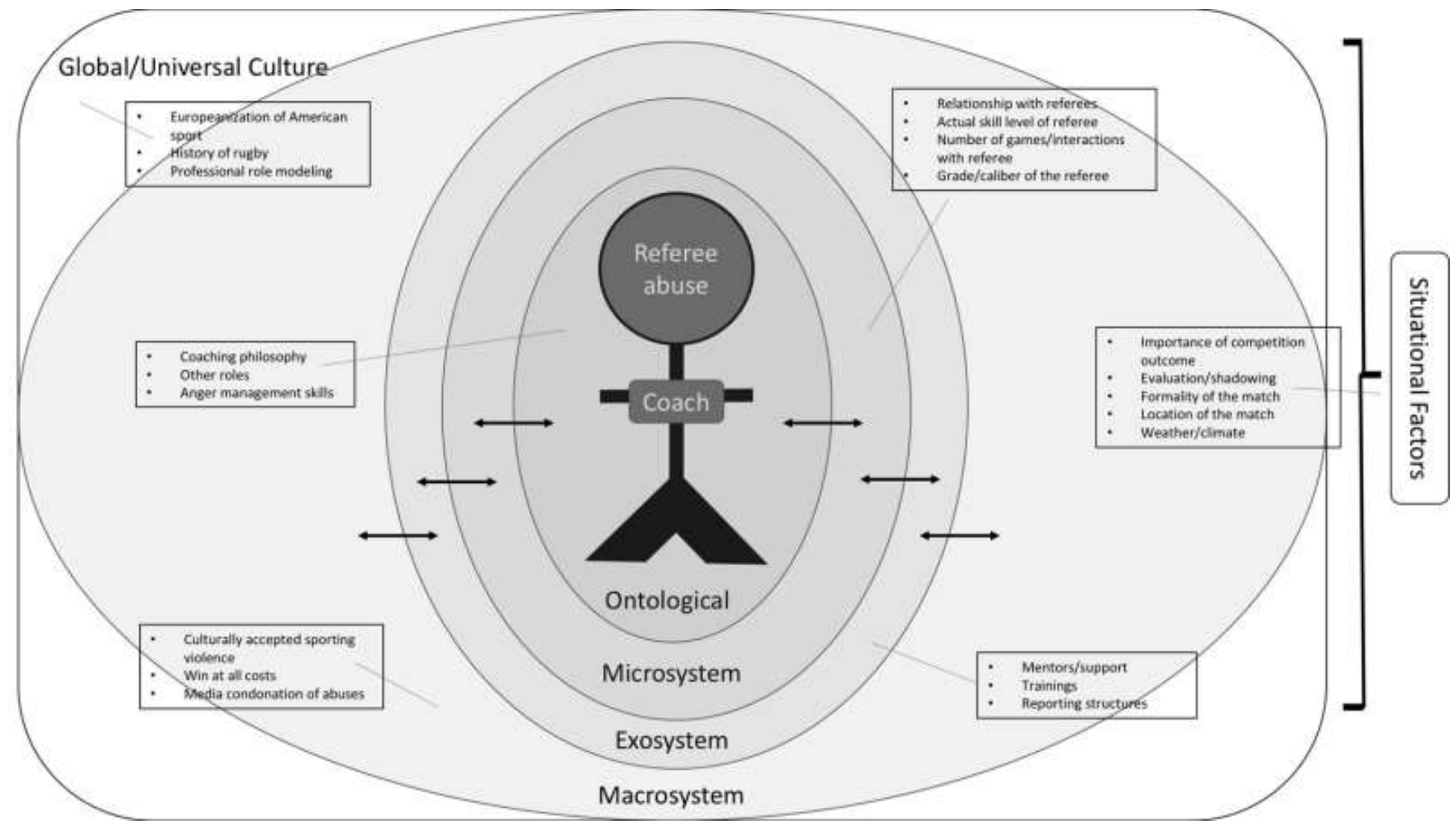

Figure 3. Conceptualization of referee abuse from the perspective of the rugby coach. 
The ecological-transactional framework aligns with the study of referee abuse from the perspective of the coach as it sets the stage for exploration of ecological factors that may impact one's proclivity toward or understanding of referee abuse. The ecological-transactional framework is bound by four categories of ecological factors, as shown in Figure 3. The first level of consideration, ontological development, encompasses the characteristics of the individual studied that may explain his or her tendency toward a behavior. In the case of this study, the researcher explored personal tendencies toward referee abuses or personal propensity toward specific perceptions of referee abuse. Such personal factors may have included an individual's own experiences with abuse or other roles held. For example, if rugby coaches have also held the role of referee, their perceptions of referee abuse or their likelihood to participate in abuse behaviors may be impacted.

Beyond coaches' internal processes and predispositions, the interrelational factors between the coach and referee may also impact their perceptions of abuse or abuse behaviors. In the ecological-transactional model of vulnerability, this is considered the microsystem level. Here, perceptions of power or authority may impact the rationalization of abuses. The relationship between the coach and the referee may determine the way the coach reacts to the referee. The coach's proclivity toward referee abuse may also be impacted by his or her perceived skill of the referee or the caliber of the referee (as determined by the referee's grade with USA Rugby's hierarchy). Both the perception of skill and the referee's grade denote a level of competence that may serve to deter abusive behaviors. However, it may increase abusive behaviors if the coach finds 
the official's refereeing to be of lower quality than anticipated. Further, the interactions between these two individuals (coach and referee) will also be predicated on the amount of time they spend in close proximity. In the case of sport, this proximity will likely be determined based upon the number of games that a referee has officiated while a specific coach is coaching. Thus, it is anticipated that if a coach perceives a referee to be poor in an initial meeting, the chances of referee abuse are enhanced in subsequent meetings. Conversely, if a coach perceives a referee to be exceptional, the instances of abuse in future outings will likely be low. The interactions between a coach and referee may impact the likelihood of abuse behaviors or may impact a coach's perceptions of abuse behaviors toward a particular referee.

Beyond the direct interactions between the coach and referee, the exosystem level considers the impact of a sporting community. Within the context of the sporting community, coaches may look to mentors, other sports coaches, or other prominent members of the sporting community to guide their behaviors and perceptions. The normalization of referee abuse within a specific sporting community may suggest abuse as an acceptable practice (Wolfson \& Neave, 2007). Further, tolerance exhibited by sporting organizations in the form of minimal repercussions for reported abuse increases the vulnerability of the referee. In the context of this study, the coaches' communities may have an impact on referee abuse perceptions or behaviors. The collegiate rugby coach exists within a number of communities, including the coaching community, the rugby community, and the collegiate community. The collegiate sporting community may expect a certain level of behavior from coaches and players, thus influencing the behavior of coaches when it comes to referee abuse. The rugby community may impact a coach's 
abuse behaviors based on the availability of training and reporting structures. If coaches are trained to understand referee abuse and provided an opportunity to report poor officiating, that may have an impact on their perceptions of referee abuse or their own abuse behaviors. Because each of these communities may have different and varying levels of impact on the coaches' behaviors and perceptions of referee abuse, this category of factors helps to create a holistic picture of referee abuse through the lens of the rugby coach. Thus, the ecological-transactional framework creates a guide for such exploration.

The final component of the ecological-transactional model is the macrosystem level; here, the sporting community at large is considered. Within the sporting community, aspects of sport culture may impact both the perceptions and behaviors of a coach and the vulnerability of a referee to abuses. Culture is often explored through the interconnectedness of meaning, materiality, and social practice (Malinowska \& Lebek, 2016), and one's culture is defined by the values, behaviors, and traits within a particular region (Hofstede, 1980; Huffman \& Hegarty, 1993). Individuals within a particular culture or who identify with a culture will adopt the social norms and established traits of the culture and may subsequently enact culturally accepted behaviors. Every social group manifests its own culture, and sport is no different. Sporting culture in America tends to normalize violence within sport (Fields et al., 2007) and to promote the meaning of sport to such great heights that a win-at-all-costs environment is often created. Stirling and Kerr (2014) have also indicated that "performance-based values in sport may lead to the prioritization of athletic performance over the well-being of an athlete, thus increasing the athlete's vulnerability to emotional abuse" (p. 131). The same may apply to officials in rugby. When rugby coaches prioritize athletic performance and success over fairness 
and equality, the referee may become more vulnerable to abuse. The media may have also helped to perpetuate a narrative that understands violence as part of sport and that abuse behaviors are to be accepted (Stirling \& Kerr, 2014). In American sport, this is exemplified in a television advertisement created by the National Football League (NFL) in which Mike Zimmer (coach of the Minnesota Vikings) writes repeatedly on a whiteboard, "I will be nicer to the officials." The advertisement, which is part of the NFL's character program, highlights the expectation of referee abuse within the American sporting culture. Rugby coaches' existence within the cultural norms of American sport and their exposure to such advertisements may impact the way in which they perceive referee abuse behaviors. In the rugby sporting context, the larger social norms of American sport may impact a coach's perception of or resultant referee abuse behavior. This final level of the ecological-transactional framework allows for exploration of social norms and sporting norms when considering referee abuse in rugby.

Beyond the current scope of the ecological-transactional framework is the idea that situational factors may also impact one's propensity toward a specific behavior or perception. While exploring abuse in the coach-athlete relationship, Stirling and Kerr (2014) reported that the frequency of abusive behaviors correlated with the intensity and importance of the competition. As the competition became more important, the instances of verbal abuse increased (Stirling \& Kerr, 2014). It has thus been concluded that one's propensity for abusive behavior is heightened by situational factors (Stirling \& Kerr, 2014). In considering referee abuse, it is likely that a similar pattern will emerge. Coaches' behaviors tend to become more egregious based on their perceptions of the intensity of the competition or situational factors such as their perception of a referee's 
ineptitude. In their research, Stirling and Kerr (2014) addressed situational factors within the ontological level of development, suggesting that one's reaction to situational factors was an innate predisposition. In the current study, since referee abuse often occurs simultaneously with numerous situational factors, the researcher had to consider whether or not those factors were truly confined to the ontological level of development or if their impact was more wide-reaching.

Further, unique to the study of coaches' perceptions of referee abuse in American rugby is the idea that there may be an international or historic level of ecological consideration that may exist. Because rugby is not originally an American sport, the ways in which American rugby coaches idolize their European or international counterparts or mimic their behaviors may impact referee abuse behaviors in American collegiate rugby. Internationally, professional rugby competitions are common, thus providing models and mentors for university level coaches. Further, the derivation of rugby values, including that of respect for the referee, from the beliefs of Muscular Christianity in Victorian-era England may or may not align with or even enter an American rugby coach's realm of understanding. As a result, the ecological transactional model put forth by Cicchetti and Lynch (1993) may categorize a significant portion of the factors that impact a coach's perception of referee abuse but may not be all-encompassing.

Because referee abuse can be so closely tied to previous utilizations of the ecological-transactional framework of vulnerability, it is reasonable to conjecture that the factors impacting the vulnerabilities of the referee may exist within the categories presented in the ecological transactional model of vulnerability. There are numerous ecological factors that may impact a coach's perception of referee abuse or their abuse 
behaviors. These factors may be able to be organized into the ontological, microsystem, exosystem, and macrosystem levels of analysis. As such, the ecological transactional model creates a template to organize such factors and helps to later position the data. As the data emerge from the research, the conceptual framework is likely to change in response to the information garnered from the participants, expanding or contracting the existing model. Allowing data to drive any outcomes is of utmost importance to a phenomenological study. Becker (1986) cautioned that trying to mold your data to fit a pre-defined framework may cause the researcher to overlook other conceptualizations. Thus, conceptual frameworks such as the ecological-transactional model are intended to guide phenomenological research rather than test hypotheses that may be encompassed within. Becker (1986) further proposed abandoning ideological assumptions during data analysis in order to illuminate new ways of framing the phenomenon. Viewing the data with an open mind allowed the researcher to consider the contribution of new conceptualizations or the ways in which an existing framework may or may not apply to a specific context or participant group.

\section{Conclusion}

Referee abuse appears to be a pressing issue facing sporting organizations at all levels. Continued abuse toward referees has the ability to contribute to referee stress, performance issues, and referee recruitment and retention concerns.

It is difficult to imagine any other modern western industry in which abuse, intimidation and violence of this kind would be regarded as legitimate instruments of managerial control; indeed, outside the relatively closed social world of professional [sport], these techniques would almost universally be regarded not just as bad management practice but would almost certainly result in cases being brought to industrial tribunals and might also result in criminal prosecutions. (Kelly \& Waddington, 2006, p. 154) 
Yet sporting professionals have done little to empower sporting participants to act as agents of change. Beginning with coaches, who have the ability to holistically impact the perceptions of referee abuse and model behavioral changes, this study took the first step in understanding perceptions of referee abuse and providing potential solutions to decrease its prevalence.

The literature provides support for this study as coaches are shown to be key influencers in the sporting structure (Mastroleo et al., 2012; Perna et al., 1996). Coaches have the power to influence participant attitudes and behaviors, and thus the understanding of their perceptions regarding referee abuse is key to the development of a holistic view of referee abuses and potential solutions. Therefore, this study attempted to fill a gap in the literature by examining referee abuse through the lens of collegiate rugby coaches. 


\section{CHAPTER III}

\section{METHODOLOGY}

Chapter three is comprised of five sections: (1) Research Design, (2) Methods, (3) Data Analysis, (4) Trustworthiness and Dependability, and (5) Summary. The primary purpose of the study was to explore collegiate coaches' perceptions of referee abuse and to better understand the factors that contribute to these perceptions. Additionally, the study sought to explore potential solutions, generated by coaches, to alleviate the problem of referee abuse within the sport of rugby at the collegiate Division I-A level.

Crotty (1998) suggested that there are four requisite elements necessary for any qualitative research process: (1) Epistemology, (2) Theoretical Perspective, (3) Methodology, and (4) Methods (see Figure 4). The first element, epistemology, explores the researcher's philosophical assumptions about knowledge and how it is developed. Understanding one's epistemology helps to illuminate the beliefs that underpin a researcher's understanding of the world and how they may be incorporated into the present study. The second element in the qualitative research process is one's theoretical perspective. A theoretical perspective is the researcher's approach to understanding the world and making sense of it (Crotty, 1998). Both a researcher's epistemology and theoretical framework help to justify the chosen methodology. A methodology, or plan of action, expresses how one's research was designed and links to the use of a particular set 
of methods. The fourth element, methods, includes techniques or tools that are used to gather data and explore a research question (Crotty, 1998). These four elements are used as scaffolding to outline the qualitative process in this study.

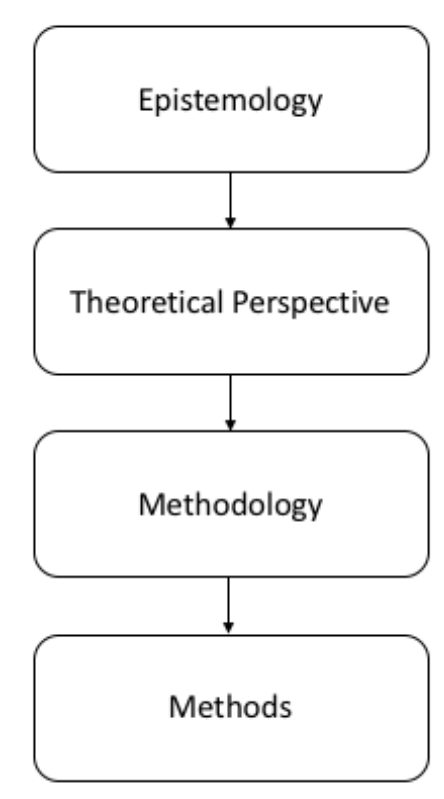

Figure 4. Crotty's (1998) model for qualitative research design.

\section{Research Design}

In designing a qualitative study, one must begin by understanding the researcher's theory of knowledge and exploring how one distinguishes truth from opinion (Schwandt, 2015). The way that one views the world is known as an epistemology. Researchers' epistemology deals with their understanding of the "nature of knowledge, its possibility, scope and general basis" (Hamlyn, 1995, p. 242). Essentially, researchers' epistemology serves as an explanation of how they know what they know (Crotty, 1998). The researcher in this study held a constructivist epistemology, suggesting there is no objective truth but rather that truth is subjective and comes into existence based on one's interactions with the world (Crotty, 1998). Thus, meaning is constructed rather than 
discovered and comes into existence as subjects and objects interact (Crotty, 1998).

Without a mind, there can be no creation of meaning. The belief that meaning is constructed through interaction and transmitted in a social context explains the perspective of the researcher who drove this study. Coaches' perceptions of referee abuse are not absolute or objective; they may be informed by experiences, values, situational factors, and societal norms. A researcher who ascribes to a constructionist epistemology perceives there to be an importance in understanding that every research participant has prior experiences, knowledge, skills, values, and beliefs that impact the way in which they construct meaning from a situation (Hein, 1999). These perceptions were then used to ascribe meaning to referee abuse within the sporting structure. The epistemological foundation (in this case constructivism) drove the theoretical perspective that served as the lens through which the research was viewed.

Based on the constructivist epistemology held, an interpretivist theoretical perspective was utilized to explore the stated purpose of the research study. An interpretivist approach to qualitative research focuses on meaning and seeks to explore an issue from a number of perspectives with the goal of deeper understanding (Creswell, 2013). The interpretivist theoretical perspective was dependent on constructive epistemology as the researcher must interpret the participants' experiences based upon the subjective meanings that they have constructed (Goldkuhl, 2012; Silverman, 1970). Because of the strong focus on meaning, interpretivism allowed the individual experiences of the rugby coaches to guide and be the focus of the research. The subjective meanings of a coach's experience with referee abuse were found to be varied 
and multiple. As such, this research sought to explore referee abuse through the lens of the coach's experience, utilizing their viewpoints to derive any conclusion.

A researcher utilizing an interpretivist framework understands "subjective meanings [to be] negotiated socially and historically" (Creswell, 2013, p. 25). Thus, an individual's perceptions are socially constructed via interactions with others and are impacted by the social norms of the environment in which the individual operates. These perceptions are then interpreted in order to ascribe meaning. Coaches' perceptions of referee abuse cannot be adequately accomplished within a vacuum, for these perceptions exist within the larger sporting structure and the sporting environment in which the coaches participate. Thus, an interpretivist theoretical framework allowed individual meanings to guide this study while considering the larger context in which those meanings were derived. An interpretivist theoretical perspective aligns well with the aforementioned conceptual framework, which sought to explore referee abuse within one's larger ecological context. The ecological-transactional conceptual framework (Cicchetti \& Lynch, 1993) oriented this research in a way that allowed for the interpretation of experiences within varying social contexts, driving the constructs and concepts to be considered as part of the study. To explore the concept of referee abuse within a coach's environment and to allow for the interpretation of a coach's experiences within a social and historical frame, a qualitative methodology and phenomenological approach were utilized.

Methodology is defined as the process or design that underlies a researcher's choice of methods (Crotty, 1998). When conducting qualitative research, there are numerous methodological frameworks that can be utilized to guide the study, including 
ethnography, phenomenological research, grounded theory, and heuristic inquiry. The researcher chose a phenomenological methodology to guide the selection of interview methods and shape their use throughout the study.

Phenomenology is largely credited to Edmund Husserl, who advocated that reality consists of phenomena and how we experience those phenomena. Phenomena are described as things as they appear, thus the term phenomenology, which is "derived from the Greek word phainomenon, meaning appearance” (Sarker, 1994, p. 17). Husserl believed that individuals' perceptions accurately represent their consciousness (Fouche, 1993) and that everyday consciousness could be described while suspending, or bracketing, any preconceived notions. As a result, phenomenology is often descriptive rather than explanatory and provides a concise description of the way things appear. Stewart and Mickunas (1990) expressed four philosophical perspectives intrinsic to phenomenology: (1) a return to traditional tasks of philosophy—searching for wisdom, (2) a philosophy without presuppositions [epoche] — suspending judgments until they can be more foundationally sound, (3) intentionality of consciousness-one's reality is directly related to one's consciousness of an object, and (4) refusal of subject-object dichotomy - reality can only be perceived from the meaning that an individual derives from their experiences. These philosophical perspectives broadly encompass the two primary forms of phenomenology, hermeneutic phenomenology, and transcendental phenomenology. According to van Manen (1990), phenomenology is not merely a description but an interpretation of the meaning of lived experiences by the researcher. Here, van Manen follows the logic of Martin Heidegger, asserting that interpretation necessitates personal awareness, and therefore, it is impossible to completely negate or 
bracket our experiences related to a particular phenomenon. Conversely, transcendental phenomenology, as understood by Moustakas (1994), requires the bracketing of one's experiences, though Moustakas admits that this is an imperfect process.

Thus, this study utilized a hermeneutic phenomenological approach that aligns with the constructivist epistemology and interpretive theoretical perspective adopted by the researcher. The researcher believes that no phenomenon can exist in a vacuum, and thus it is difficult to fully bracket out any preconceived notions regarding the phenomenon. Despite this, the researcher was sure to identify any biases and conceptual underpinnings that may bear reference to the study. Ultimately, through the phenomenological inquiry, the researcher sought to illuminate the perceptions of rugby coaches toward referee abuse, as these perceptions represent the reality of referee abuse in this context. A further goal of this phenomenological research was to determine the factors that underlie individuals' perceptions and develop actionable solutions. A phenomenological approach allowed the researcher to deconstruct the human experience and to ascribe meaning to those experiences (Creswell, 2013) as they relate to a particular object. In this case, the researcher explored the ways in which coaches have experienced referee abuse in the sporting structure as the object of intention. Because phenomenology is particularly effective at providing an in-depth look at motivations and behaviors (Crotty, 1998), the approach afforded the research the opportunity to elucidate the current state of referee abuse as experienced by the collegiate rugby coach. A rugby coach's insights are based on the individual's own experiences and perceptions rather than assumptions or conventional wisdom. By exploring referee abuse through the lived experience of a coach, the individual is able to challenge or affirm social and structural 
norms and attempt to "grasp the very nature of the thing" (van Manen, 1990, p. 170).

“The basic purpose of phenomenology is to reduce the individual's experiences with a phenomenon to a description of universal essence" (van Manen, 1990, p. 177). Thus, instead of generating a list of socially acceptable responses, a phenomenological approach allowed the researcher to clarify the underlying assumptions that surround referee abuse and to find "commonalities across participants to see how lived experiences relate to a phenomenon of interest" (Hays \& Singh, 2012, p. 50). The exploration of lived experiences through the use of a phenomenological methodology dictates the use of interview methods in order to uncover the truth in the study.

\section{Method}

\section{Participants}

The current study explored referee abuse within the sport of rugby. Rugby was selected as the sport of study because rugby has often been referred to as a gentleman's game where respect is afforded to the referee. The historical underpinnings of rugby and the close connection to Muscular Christianity create a game that is currently governed by core values, including integrity, passion, solidarity, discipline, and respect (World Rugby, 2014). These intrinsic elements of rugby suggest that the social norms for referee abuse in rugby may differ from other sports. Within the sport of rugby, USA Rugby collegiate coaches in the Division I-A men's competition were selected as the sampling frame. Individuals who were registered members of the USA Rugby National Governing Body at the time of the study and serve as a men's Division I-A collegiate rugby coach were eligible to participate in this study. USA Rugby currently lists 70 men's Division I-A collegiate programs. USA Rugby is the National Governing Body for the sport of rugby 
in the United States, which provides authenticity and credibility to the coaches' participation in rugby. Further, because men's Division I-A rugby is the highest level of the collegiate rugby game, the stakes for competition are typically higher. High-stakes competition may have an impact on the breadth and depth of referee abuse that coaches have experienced. Additionally, coaches at the Division I-A collegiate level have a requisite amount of experience in rugby and coaching. Based on the experience that the Division I-A level necessitates, these coaches were likely to have worked in rugby for a number of years and thus would have greater experience to draw from when discussing referee abuse within the rugby structure.

Oftentimes, it is not feasible to collect data from the entirety of a target population; because of this, a subset of this population, or sample, is selected for study (Fraenkel, Wallen, \& Hyun, 2011). In this case, participants were recruited through purposive sampling, which is defined as having been selected based on the characteristics of the population and the objectives of the study (Palinkas et al., 2015). In a phenomenological study, it is "essential that all participants have the experience of the phenomenon being studied" (Creswell, 2013, p. 144). Here, it was essential that all coaches had witnessed or participated in referee abuse within the sport of rugby.

Based on the use of the inclusion criterion in selecting a sample for this study, this type of purposeful sampling is known as criterion sampling. Criterion sampling requires the selection of selecting cases that "meet some predetermined criterion of importance" (Patton, 1987, p. 56). In this case, participants were recruited and selected based on three criteria: (1) registration as a coach with USA Rugby, (2) currently coaching at the men's Division I-A level (as defined by USA Rugby), and (3) having borne witness to or 
experienced referee abuse within the game of rugby. According to Moustakas (1994), the researcher should collect data from individuals who have experienced the phenomenon; thus, the third inclusion criterion is of great importance. It is also important that the participants were registered as coaches with USA Rugby in order to verify their association with the sport. Further, the coaches must be currently coaching at the Division I-A level as it is the most prestigious collegiate competition available and has often been seen to set the standard for policies and structures at other levels of competition. Being a current coach, as signified by one's coaching membership with USA Rugby and one's association with a Division I-A men's rugby team, ensured that the participants were able to provide contemporary accounts of their experiences with referee abuse. While many retired coaches may have had similar experiences, they would not have had the same ability to reflect on referee abuse in the present.

Because there is a limited number of people who could serve as a primary data source to examine this phenomenon, criterion sampling allowed for selection and inclusion as a result of the participants' shared characteristics (Creswell, 2013; Palinkas et al., 2015). Thus, purposeful criterion sampling provided access to "key informants in the field who can help in identifying information-rich cases" (Suri, 2011, p. 67). When speaking with these coaches regarding referee abuse, the interviewer sought to understand what they experienced and how they experienced it. That allowed the researcher to develop a composite description of the experience of referee abuse in order to portray the essence of the phenomenon (Moustakas, 1994).

Coaches in this men's Division I-A collegiate membership category were recruited through an email sent by Kevin Battle, USA Rugby's DI-A Commissioner, to 
all registered men's Division I-A head coaches. Those interested in participation were asked to complete a brief demographic survey (See Appendix A) that began with a consent from and asked questions including the number of years coaching rugby, the number of years coaching DI-A rugby, and questions confirming the aforementioned inclusion criteria. Fifteen coaches, including five assistant coaches and 10 head coaches, met the inclusion criteria to be included in the study. This sample size was adequate to explore the phenomenon of referee abuse in rugby, as Guest, Bunce, \& Johnson (2006) found that 12 interviews of a homogenous group would allow the data to reach saturation. Crotty (1998) also indicated that a phenomenological study would require five to 25 participants. Morse (1994) noted that at least six participants would be necessary, and Creswell (2013) posited that a phenomenological study requires " 3 to 4 individuals to 10 to 15 individuals" (p. 78).

In phenomenological research, it is important to ensure that the essence of the issue has been adequately described by the participants in the study and the study is able to be replicated (Fusch \& Ness, 2015). Saturation is an important piece of qualitative research, including in phenomenological inquiries, but must be appropriately defined within the study. In this case, the researcher sought "saturation of knowledge" (Bertaux, 1981, p. 37), meaning that the researcher had come to recognize patterns in the experience of participants and that additional interviews were serving to confirm what the researcher had previously sensed. The researcher looked for saturation of topics discussed between interviews in order to determine if additional participation should be interviewed. 
Once topics discussed had become redundant and new information was no longer emerging from the interview sessions, the researcher considered this the point of data saturation.

\section{Demographic Data}

Of the initial 20 participants who expressed interest in the study, two were excluded based on the requisite inclusion criteria: (1) registration as a coach with USA rugby, (2) currently coaching at the men's Division I-A level (as defined by USA Rugby), and (3) having borne witness to or experienced referee abuse within the game of rugby. Another three participants expressed that they would schedule their first interview after the collegiate playoffs had concluded. Based on the lack of response to follow-up emails sent by the researcher, these coaches were removed from the study. Thus, of the initial 20 individuals who had expressed interest in participation, 15 coaches completed the study. All 15 participants were able to complete two interviews for a total of 30 completed interviews. The interviews ranged in length from 36 minutes to 68 minutes, with the average first interview lasting 51 minutes and the average second interview lasting 53 minutes. Demographics were presented through the following sections: programmatic demographics, rugby roles, and personal demographics.

Programmatic demographics. Collectively, the 15 coaches represented 11 of the $71(15.5 \%)$ Division I-A collegiate rugby programs in the United States. The rugby coaches included in this sample represented programs in six of the eight Division I-A collegiate rugby conferences, including the Big10, Cal, East, Liberty, Red River, and West Conferences. The conferences and therefore the programs are geographically spread across the country, representing institutions from the East Coast to the West Coast of the 
United States. Eight public institutions and three private institutions were represented, with institutional enrollment ranging from 1,500 to more than 50,000 .

Rugby roles. Despite the relative youth of the Division I-A (DI-A) league structure, which started in 2011, the coaches in this sample averaged 4.6 years of coaching participation at the elite collegiate level (DI-A). The coaches also had significant experience coaching rugby (at any level) with an average coaching tenure of 18.1 years and coaching careers that ranged from four to 35 years in length. In terms of the coaching role held, five of the participants held the role of men's Division I-A collegiate assistant coach, and 10 were men's Division I-A rugby head coaches. The head coaches averaged a total coaching tenure that was 11.9 years longer than the assistant coaches and was, on average, 1.5 years longer at the elite Division I-A level. In addition to their coaching roles, ally coaches interviewed had played rugby. Several had also participated on a national team representing their country of origin.

In addition to their previous playing and coaching careers, nearly every coach noted that they maintained at least one other professional role. For some, this was fulltime employment outside of rugby; for others, this was another role within the rugby community. Just more than half of the coaches who participated in this study ( 8 of 15) were also certified as referees. Two of those who were not certified as referees mentioned consciously avoiding officiating because they did not feel that it aligned with their strengths. Another two coaches, who noted that they were not referee certified, mentioned that although they were not certified, they did occasionally officiate to help their teams or youth levels of the game. 
Personal demographics. Of the 15 participants interviewed, 14 were male and one was female. The male-to-female ratio of coaches was consistent with the coaching demographic and was therefore expected in men's collegiate rugby since at the time of the study, no female head coaches existed at the men's Division I-A level. However, it is important to note that women served as officials for the Division I-A men's competition. Looking beyond gender of the coaches who participated, five nationalities were represented, 10 coaches were born in the United States, and five coaches were born outside of the United States. Holistically, the group of coaches included individuals from four continents: Asia, Europe, North America, and Oceania.

The 15 Division I-A collegiate rugby coaches offered a vast array of rugby-related experiences, which provided a wealth of knowledge. As such, representative, verbatim interviewee responses were included in the study to retain the voices of the participants. Participant responses were identified by an assigned pseudonym, which is designated by a set of initials. Responses were stripped of any additional identifying information. The participants' reflections upon and understandings of referee abuse in rugby are detailed in the subsequent sections.

\section{Data Collection Procedures}

The approval of the university Institutional Review Board was sought preceding data collection (see Appendix B). Upon receipt of approval, the researcher worked with DI-A League Commissioner Kevin Battle to disseminate an email (see Appendix C) to the Division I-A collegiate rugby coaching population, soliciting participation in the study. The initial contact email asked that those who were willing to participate fill out a brief screening questionnaire in order to confirm their interest and to ensure that they 
meet the sampling criteria: (1) registration as a coach with USA Rugby, (2) coaching at the Division I-A level (as defined by USA Rugby), and (3) having borne witness to or experienced referee abuse within the game of rugby. If the individual did not meet any of the sampling criterion, they were thanked for their participation and removed from consideration for the study. In order to solicit additional participants, Battle also sent a follow-up email to the DI-A coaching listserv. A subsequent follow-up email was sent by the investigator.

Of the initial 20 participants who expressed interest, two were excluded since they did not meet the inclusion criteria. Another three participants failed to schedule an interview with the researcher after completing the pre-screening questionnaire. Thus, two interview sessions were scheduled with the remaining 15 participants, and each participant was emailed a consent form for their review. A total of 30 interviews were completed. Oftentimes, phenomenological studies consist of multiple in-depth interviews with participants (Polkinghorne, 1989). Completing multiple interviews provided a structure in which the researcher could clarify previously collected data and probe further to extrapolate additional information (Glaser \& Strauss, 1967) regarding one’s experience with the phenomenon of referee abuse. Utilizing two semi-structured interviews in the data collection process also allowed the participant to become more familiar with the researcher, creating a safe environment to share experiences (Knox \& Burkard, 2009). Further, utilizing dual interviews in this study increased the depth and meaning that the researcher was able to extract from the interviews and allowed the interviewer to explore additional considerations from the first interview during the subsequent contact (May, 1991). The interviews took place by phone, which allowed the researcher to access 
coaches from all over the United States rather than limiting the pool of participants to a specific geographical region. Though there are few research studies that compare inperson interviewing to phone interviewing, Siemiatycki (1979) found that the two procedures were comparable in terms of the quality of data obtained and thus suggested that the additional cost of in-person interviewing is unwarranted. Beyond that, the nonverbal cues that a researcher introduces during an in-person interview may impact the participants' responses and create a potential for bias (Marcus \& Crane, 1986; Musselwhite, Cuff, McGregor, \& King, 2006). As a result, phone interviews were utilized in this study, with the first interviews being completed in late March of 2018 and continuing through May of 2018.

Two semi-structured interview guides were utilized to collect data. The creation of the first interview guide (see Appendix F) was guided by the ecological transactional model of vulnerability (Cicchetti \& Lynch, 1993). From Cicchetti and Lynch's (1993) model, the researcher was able to discern interview questions regarding factors that may influence one's perceptions of referee abuse or propensity to engage in referee abuse. Moustakas (1994) also advised that phenomenological studies using interviews should include two broad questions regarding: (1) experiences with the phenomenon and (2) contexts or situations that influenced these experiences. As such, the interview guide included the following broad questions regarding the phenomenon of referee abuse (the full interview guide may be found in Appendix F):

1. Can you please describe any instances of referee abuse that you have seen or experienced during your time in rugby?

2. What factors (contexts or situations) have influenced or affected your experiences with referee abuse? 
The first interview also examined the coaches' perceptions regarding potential solutions to the problem of referee abuse, should they feel that solutions are warranted. Data collected from the first set of interviews then helped to inform the creation of the interview guide for the second interview (as seen in Appendix G).

Because the topic of referee abuse could have been difficult for some coaches to discuss and some element of social desirability bias may have existed, it was important to fully engage the participants in the conversation. The semi-structured approach was thus selected to facilitate the natural flow of conversation, ensuring that additional insights would not be missed due to the rigidity of questioning (Creswell, 2013). Further, it has been suggested that semi-structured interviewing can "incorporate both open-ended questions and more theoretically-driven questions, eliciting data grounded in the experience of the participant as well as data guided by the constructs in the particular discipline within which one is conducting research" (Galletta, 2013, p. 45). When utilizing semi-structured interviewers, the interviewer asks all of the questions included in the interview guide but may further pursue particular topics that emerge from the questioning for each interviewee (Hill et al., 2005; Hill, Thompson, \& Williams, 1997). Such was the case for the semi-structured interviewing process utilized in this study. Using the same interview frame for each participant enabled the researcher to make comparisons across cases, and semi-structured interviews also provided the researcher with the opportunity to vary the sequence of questioning to enhance the natural flow of the conversations.

Within the interview guide, the interview questions utilized were open-ended to facilitate discussion regarding the topic of consideration: referee abuse (Jacob \& 
Furgerson, 2012). Patton (1987) recommended that good research questions should be open-ended, neutral, and clearly understood by the interviewee. The research questions were framed using terminology common to the participant and have been checked by experts in the field of rugby to ensure clarity of questions. Using open-ended questions allowed the researcher to communicate in the interviewee's own vocabulary (Britten, 1995 ) and to ensure clarity and understanding. Open-ended questions provided the participants with the opportunity to infuse their experiences into the discussion (Silk, 2005). Allowing participants to use their own words and to ascribe meaning to their experiences as they saw fit originates with the phenomenological methodology chosen. As the participants shared their stories, the researcher encouraged participants to elaborate on any short answers in order to fully explore the topics that arose. To do so, probes were utilized as needed (e.g., Can you please explain that further? Are you able to provide an example? and/or Is there anything else?) (Peters \& Halcomb, 2015).

Prior to data collection, the interview guide was field tested to ensure the appropriateness and relevance of the questions contained within. The interview guide was field tested by three experts in the field of rugby: James Wilber, the USA Rugby National Competitions Manager for Collegiate Events; Richard Every, the High-Performance Referee Manager at USA Rugby; and Chase Langdon, the former Coaching Department Manager at USA Rugby. Field testing of the interview guide helped to ensure that interview questions were relevant and appropriate while enhancing readability and comprehension (Mertens, 2010; Nevo, 1985). Based on the results of the field test, the interview guide was refined, and minor changes in question content and order were made prior to implementation with study participants (de Vaus, 2014). The final version of the 
interview guide was then utilized to conduct pilot testing with two individuals external to the study, one who had a background in rugby and the other in coaching. Pilot testing allowed for the determination of timing and to evaluate the need for additional lines of questioning (Creswell, 2013; Galletta, 2013; Jacob \& Furgerson, 2012; Turner, 2010). When pilot tested, the interviews took between 46 and 52 minutes, and no additional lines of questioning were added.

Each of the participants' first interviews began with a review of the informedconsent form, and participants were asked to verbally reconfirm consent to participate (see Appendix E). Data were then collected through the use of the two aforementioned semi-structured interview guides. The interviews were audio-recorded, and the researcher maintained a reflective research journal throughout the process to ensure the replicability of the process (Henwood \& Pidgeon, 1992). The researcher's journal consisted of descriptive information, including dates, times, and reflective information; and thoughts, questions, and concerns that arose during the interviews. Maintaining a reflective journal helped ensure the replicability of the study while also providing the researcher the ability to be transparent about any concerns or biases that may have been encountered during the research (Ortlipp, 2008).

\section{Data Analysis Procedures}

As a means to protect the identity of the participants, each participant was assigned a set of randomly-generated initials to serve as a pseudonym. The pseudonym was then utilized for the remainder of the study and was used in the presentation of results. The data collected from the semi-structured interviews were audio recorded and transcribed verbatim. Verbatim transcriptions helped to ensure that the essence of ideas 
put forth by the participants was captured and that the voices of the individuals were preserved (Creswell, 2013). As the transcriptions were completed, inductive thematic analysis was utilized, as there is no prescribed method for data analysis within hermeneutic phenomenology (Green \& Holloway, 1997). Kafle (2013) suggested that although there are no analytic requirements, the following five research activities serve as recommendations: (1) commit to an abiding concern (phenomenon), (2) utilize an oriented stance toward the question, (3) investigate the experience as it is lived, (4) describe the phenomenon through writing and rewriting, and (5) consider both the parts and the whole. Inductive thematic analysis, as outlined by Braun and Clarke (2006), appropriately addressed these recommendations and provided a structure for the derivation of meaning from the interview data.

To pursue an inductive thematic analysis, the researcher (1) became familiar with the data, (2) generated initial codes from the open-ended responses of the participants, (3) assessed the codes to create themes, (4) reviewed developed themes, and (5) defined and named created themes (Braun \& Clarke, 2006). The first step in the data analysis process, becoming familiar with the data, included transcription of all interviews by the researcher. The researcher transcribed each interview verbatim, and the process of transcription allowed the researcher to become initially familiar with the data. Following transcription, the interviewer provided all participants with a copy of their transcribed interviews by email. The participants had the opportunity to review the transcriptions and ensure that the data collected were correctly captured by the researcher. The participants' review of transcripts served as a means of member checking and helped to build credibility with the data (Patton, 1987). Once the participants confirmed that the data 
included in their transcripts were correct, the researcher read and reread the transcripts. At that point, the researcher delved into the minutia of the data, which allowed the phenomenon to reveal itself from within. Taking the time to actively reflect on the data was foundational to the future data analysis, and it was at that point that the researcher began to make notes on potential patterns and highlight key insights provided by the participants.

In the next step of this analysis, initial codes were generated. Because the data analysis framework was inductive, codes were derived from the data rather than from a previous hypothesis or categories that had been determined a priori (Guest, MacQueen, \& Namey, 2011). To create the initial codes, the researcher worked with two external coders to ensure a thorough review of the data. To develop codes, both the coders and the researcher met and coded two interview transcripts. Preliminary codes were created, and the coders revisited each code in order to ensure that the code appropriately captured the essence of the data it would present. From this process, the researcher created a preliminary codebook. The codebook included a definition for each code that could be used in future coding, as well as sample quotes to help clarify the meaning of the codes. Using the codebook, the researcher coded the remainder of the interviews, adding new codes to the codebook as the data warranted. NVivo 11, software developed by QSR, was used to code the interviews. This software was incredibly beneficial as it provided the researcher with the ability to include demographic data for each participant interview, thus allowing the researcher to segment the codes in numerous ways. Toward the end of the coding phase, the coders were asked to code an additional two transcripts. When coding transcripts for a second time, the researcher and external coders were looking for 
intercoder agreement among the codes assigned to a particular passage based on the definition created in the codebook. There were a few inconsistencies noted in the coding of the passages. The majority of the inconsistencies were a result of multiple codes that were similar in definition or connotation. Some examples of the coding inconsistencies included the following codes: values of the game vs. spirit of the game; feedback vs. communication; and ego vs. entitlement. As recommended by Miles and Huberman (1994), at least $80 \%$ of the codes assigned to the passages in the two verification interviews matched between the coders and the researcher. Despite this, the researcher did go back and update the codebook, combining or clarifying the codes that caused many of the coding inconsistencies. The researcher also used NVivo 11 to recode or combine codes and ultimately group and categorize data during the analysis.

In the third step of the data analysis process, the codes were organized into preliminary themes. A theme is defined as a coherent integration of the disparate pieces of data that constitute the findings (Sandelowski \& Leeman, 2012). Here, the researcher looked for patterns between the codes and coded data, categorizing and grouping the data. The tentative themes were often visualized through drawings/maps. A thematic map helped to create a visual representation of the codes and subsequently developed themes (Braun \& Clarke, 2006). The goal of such a process was to ensure that clear and distinct themes were created during this review stage. After many iterations, the tentative themes were reviewed by Dr. Jacob Tingle, an external reviewer in the field of Sport Management. Tingle is an expert in the study of referee recruitment and retention, inclusive of issues related to abuse. Thus, his review of themes provided additional insights that aided the researcher in the naming of themes and confirming their 
relationships to one another. Hermeneutic phenomenology, which focuses on the interpretation of an experience, allows for preconceptions, including existing knowledge, models, or frameworks, to be integrated into the research findings (Caelli, 2000; Dahlberg, Drew, \& Nystrom, 2008). Thus, the researcher was able to integrate generated themes with existing conceptual frameworks to appropriately position the current study within the sport management discipline.

In phenomenology, no matter the type or tradition, the idea of openness is key during data analysis. Husserl's transcendental phenomenology believed that a focus of phenomenological inquiry is to manage preconceptions by bracketing or abstaining from them. Heidegger's hermeneutic phenomenology, in contrast, aligns with the view that we cannot escape our own presuppositions; thus, from this perspective, induction relies on the researcher's willingness to remain open. "Here, the researcher must step away from initial pre-understandings to gain sufficient distance from which to critically and reflectively interrogate them" (Finlay, 2003, p. 21). Drawing from Heidegger's view of phenomenology, the researcher reflected on her own pre-understandings, frameworks, and biases regarding the phenomenon of referee abuse and utilized a reflexive perspective (Finlay, 2003; van Manen, 2003). Being reflexive means that the researcher was conscious about the ways in which the research questions and methods could impact the data or outcomes of the study throughout the duration of the study (Langdridge, 2007).

\section{Trustworthiness and Dependability}

To ensure authentic outcomes from this research study, the research had to be (1) credible, (2) dependable, (3) confirmable, and (4) transferrable (Guba \& Lincoln, 1994). Numerous procedures were adopted to ensure the viability of study results, including 
audio-recorded interviews, verbatim interview transcription, and the maintenance of an in-depth research journal that helped to ensure that the data collected and the themes garnered were as dependable and as confirmable as possible. The use of audio recording and the maintenance of a research journal provided a framework for replication and verification of this study. Additionally, verbatim transcription ensured that the participant's voice was reflected in the outcomes of the study. Beyond the collection of relevant data through strong research processes, the credibility of this research was also confirmed through peer review, member checking, and clarifying researcher bias.

Processes to verify trustworthiness and dependability of both the data and the study as a whole were utilized throughout the study to ensure that any emergent problems were addressed prior to the creation of permanent consequences (Schwandt, 2015).

\section{Peer Review}

Peer review served as an external check of the processes undertaken and outcomes established during this research study. Three external reviewers, Richard Every, Chase Langdon, and James Wilber, who are experts in the field of rugby coaching or officiating, reviewed the interview guide to ensure its usability with collegiate rugby coaches. Additionally, once the data were analyzed, an external peer reviewer from the field of sport management was utilized to challenge the assertions made by the researcher (Lincoln \& Guba, 1985) and "assess whether the findings are plausible, based on the data" (Merriam \& Tisdell, 2016, p. 250). In thematic development, the external reviewer was able to confirm many of the assertions made by the researcher and assisted in the clarification of rouge or misaligning themes. Throughout the study, peer review helped to clarify outcomes and ensure that the appropriate analyses had been used throughout the 
study. External review ultimately helped to verify thematic outcomes and thus increase the credibility of the results. Another review tool that was utilized in order to ensure the trustworthiness of the outcomes presented was member checking.

\section{Member Checking}

Member checking, also known as respondent validation, is the solicitation of feedback on emerging findings from those who participated in the study. Since the study consisted of two interviews, the researcher was able to utilize the second interview as an opportunity for respondents to validate or reject some of the initial findings. The second interview also allowed the researcher to confirm much of the information that the participants had presented in their first interviews. Maxwell (2008) noted that "this is the single most important way of ruling out the possibility of misinterpreting the meaning of what the participants say" (p. 244). Beyond the member checking utilized during the interview process, after the data had been transcribed, the researcher sent all participants a copy of their transcripts. That allowed the participants to review the collected data and note any inconsistencies. When reviewing the analyzed data, the participants were able to review the information that they had provided in order to ensure that it reflected their experiences and was appropriately representative. In addition to checking the research with members of the study, external coders were also used to ensure intercoder agreement.

\section{Intercoder Agreement}

In social settings, the traditional notion of reliability has been challenged as individual perceptions and behaviors are not static. As such, Merriam and Tisdell (2016) have suggested that in qualitative research, the important question to answer is "whether 
the results are consistent with the data collected" (p. 251) (i.e., are the results dependable?). Lincoln and Guba (1985) have conceptualized this idea as the dependability of one's results, advising that the results must make sense and that external researchers should agree with conclusions established. Intercoder agreement, in essence, measures the reliability of the coders as they identify themes and to validate the emergent constructs within the study (Ryan, 1999). To establish dependability in this study, intercoder agreement was sought to ensure that the coding process and subsequent themes garnered from this research were in alignment among the researcher and two external coders.

Armstrong, Gosling, Weinman, and Marteau (1997) suggested that a researcher must decide what is being agreed upon between coders, be it code names, coded passages, or the same passages coded in the same way. As part of this research design, intercoder agreement referred to both the names of codes and passages being coded in the same way among various coders across a fixed set of units. To create intercoder agreement in the naming of codes, the researcher and two external reviewers worked collaboratively to develop initial codes and then apply an appropriate name to each code. The collaborative creation of codes allowed the researcher to develop a codebook that included definitions of each code along with representative quotes for each code. Once all transcripts had been coded, the coders were again enlisted to code two final transcripts. At that time, the researcher and coders sought intercoder agreement among the codes that they assigned to specific passages throughout two interviews. Agreement of assigned codes was set at $80 \%$, as suggested by Miles and Huberman (1994). The alignment in coding between the researcher and external coders did meet the $80 \%$ threshold. Despite 
this, there was inconsistency in coding, and thus the researcher evaluated the quality of the conflicting codes and ultimately refined and combined these codes in order to increase intercoder agreement. Once the codes had been clarified or merged, the researcher recoded the interviews as necessary.

In addition to the credibility, dependability, and confirmability of this research, the transferability of findings must be addressed. Here, the researcher ensured that enough descriptive data were provided to allow future researchers to apply the findings to new settings. Lincoln and Guba (1985) stress that the burden of transferability truly lies with the new investigator who is looking to apply the findings in a new scenario. It is suggested that "the original inquirer cannot know the sites to which transferability might be sought, but the appliers can and do" (Lincoln \& Guba, 1985, p. 289). Lincoln and Guba (1985) have further asserted that "the only generalization is that there is no generalization" (p. 110). Based upon the constructivist lens utilized for this research, it is believed that all phenomena are context- and time-specific. As a result, the purpose of our inquiry was to expand a body of knowledge rather than to generalize the findings to all contexts. According to Denzin (1983), interpretivist epistemology also rejects generalization as the aim of phenomenological research, arguing that human interactions and experiences will always have multiple meanings. Thus, this research sought to provide descriptive data and foundational ideologies that may be applied elsewhere but do not venture to generalize the findings beyond the specific time and space of this research study. Explorative studies are not particularly generalizable but instead seek to develop general statements or hypotheses that can be tested for generality in subsequent studies, which this study has done. Beyond the generalizability of the study, the 
researcher will also clarify her own research bias in order to provide the utmost transparency regarding potential preconceptions or predispositions.

\section{Clarifying Researcher Bias}

In order to adequately address one's own bias as it relates to the topic of study, an individual must be reflective and honest. Understanding one's position and biases from the outset can help the reader understand how "the individual researcher might have arrived at the particular interpretation of the data" (Merriam \& Tisdell, 2016, p. 249). Throughout the study, it was important for the researcher to be open and aware of their own bias to ensure that the experiences of the participants are able to shine through and can assert their truth against the researcher's own fore-meanings (Gadamer, 1975). In this study, the researcher included a full researcher's stance (in the subsequent section), which will help to illuminate any biases or assumptions that may have existed within this study.

\section{Researcher's Stance}

As previously mentioned, it is of primary importance to consider the researcher's background in order to acknowledge any existing assumptions or relationships with the subject matter involved in the research. These previous experiences have the power to impact the research process and the interpretation of results (Sparkes \& Smith, 2014). Thus, in phenomenological studies, a researcher may bracket them out of the study (Creswell, 2013). This bracketing is not a matter of forgetting past experiences but rather determining experiences without engaging this previous knowledge (Fischer, 2009; Husserl, 1946). To acknowledge the innate biases of the researcher, the following descriptions detail the researcher's experience with rugby, referees, and referee abuse. 
Beginning in high school, the researcher became familiar with referee abuse. As a basketball and field hockey player, she witnessed numerous players, coaches, and spectators berating referees. Reflecting on her sporting career, the researcher found that she, too, was abusing referees in an attempt to bend the rules to meet her personal needs. Continuing into college, the researcher found that her experiences at that level were a near mirror image of those in high school. She often found her team's coaches and the coaches of other teams verbally abusing referees when calls were not favoring their team. Players were not immune to such behavior, and there were instances that the researcher recalls yelling at the referees as well.

The researcher has also served as a field hockey referee, beginning in high school and continuing through college. As such, she regularly minimized or ignored abuses from coaches and players. She also became familiar with using humor to de-escalate tense situations and the value of developing a relationship with both players and coaches. After college, the researcher went on to serve as the Referee Department Manager at USA Rugby. In this role, she was exposed to numerous instances of referee abuse. Beyond this, she spoke with many prospective referees and learned that the hostile work environment a referee experiences often made officiating not worth it. Similarly, it seemed that many referees left officiating due to the extreme pressures, stresses, and abuses that they faced. Anecdotally, these issues seemed to impact sporting organizations across the country, and when the researcher spoke with other national governing bodies, they all expressed similar concerns about the slow or non-existent growth rate of refereeing in their respective sports. The researcher no longer holds a role at USA Rugby and has never 
served as a rugby referee, player, or coach, in any capacity. She previously served as a field hockey coach and referee at both the club and high school levels.

The researcher acknowledges that these experiences may lead to personal bias that has the potential to distort research processes and data interpretation (Creswell, 2013). As such, the researcher made a point to remain open to the individual perceptions of the participants during the interview process, the coding, and the thematic analysis. Additionally, the researcher utilized both peer and member checking to validate findings and ensure that the outcomes of this study represent the perceptions of the participant rather than the biases of the investigator.

\section{Summary}

The phenomenological study completed has utilized 30 total semi-structured interviews with 15 men's Division I-A collegiate rugby coaches to (1) explore how men's Division I-A collegiate rugby coaches perceive referee abuse, (2) examine factors that contribute to these perceptions, and (3) illuminate actions that coaches believe may reduce referee abuse in rugby. From these interviews, inductive thematic analysis allowed the researcher to derive codes and, ultimately, pertinent themes. The salient themes guided the discussion, which utilized direct participant quotes to provide a rich description of referee abuse from the perspective of Division I-A rugby coaches. The outcomes of this study have been confirmed by both experts in the field and the participants themselves, ensuring that the essence of the phenomenon was appropriately captured and reported. Further, the findings from this study serve as a foundation for future referee abuse research and may help to develop strategies that deter referee abuse, thus increasing referee recruitment and retention. 


\section{CHAPTER IV}

\section{FINDINGS}

The findings resulting from the phenomenological methodology utilized in this study are an expression of the information and data articulated by the participants during the interview process. The findings represent the examination of coaches' perceptions of abuse in rugby through 30 interviews. In this chapter, the findings derived from the interview data were categorized into themes and organized by research question. Verbatim quotes from the participants were utilized to express the findings in the subsequent sections. Throughout this section, the participants are referred to utilizing the randomly generated pseudonyms. Because there were both males and female in this sample, the pseudonyms have been reduced to initials only.

\section{Coaches' Perceptions of Referee Abuse}

The first research question addressed was "How do men's Division I-A collegiate rugby coaches perceive referee abuse within the United States rugby sporting structure?" This section addresses the research question through the following three emergent themes: (1) defining referee abuse, (2) occurrences of referee abuse, and (3) outcomes of referee abuse. 


\section{Defining Referee Abuse}

The coaches interviewed found defining referee abuse within the context of rugby to be a challenge. While all 15 coaches interviewed noted that referee abuse was unacceptable in collegiate rugby, the behaviors that would be considered abuse varied considerably. A statement from AL epitomized the consensus among coaches regarding their perceptions of referee abuse: 'Number one is, you know, I don't condone abuse of anybody regardless of what they're doing." Throughout the interviews, it was made clear that there was no place for abuse of any kind, specifically referee abuse, in the sport of rugby. Whether or not the coaches had participated in abuse behaviors themselves during their coaching career, referee abuse was not to be tolerated. The difficulty, then, was defining the differences between negative behaviors and abusive behaviors. GR expressed difficulty in defining referee abuse:

Where do we draw the line between what's the tipping scale when you step beyond, you know, being agitated and being angry and into what constitutes abuse? Is negative feedback abuse? Is even shouting on the field "you missed the call sir," is that abuse? So, I think I don't know where the clear line of distinction is. Certainly, we don't want to misconstrue or call every negative action abuse, but we certainly don't want to give a pass for abusive actions.

The rhetorical questioning in GR's response indicated that these coaches considered a wide range of behaviors that they may perceive as abuse behaviors. Many hesitated when contemplating which behaviors should be defined as referee abuse. Some coaches described the definition of referee abuse as all encompassing, including anything that was not explicitly productive to the game. HS explained referee abuse as "anything that would distract the referee from his or her ability to organize and control the game." But all 15 coaches explained that physical abuse was fairly easy to define. The consensus among the 
coaches was that threatening physical harm or following through on that threat and physical contact with the referee would undoubtedly constitute abuse. EP explained:

I mean any time that you're threatening physical harm or implying physical harm that's well beyond the boundaries-I think that's crossing the line. Now I don't have to threaten physical harm with a referee to be abusive to him.... I think whenever you talk about physical harm we're talking about potential criminal action. And I would say that abuse can include up to criminal action, but it starts well before that, too.

Physical behaviors against a referee were understood to be unacceptable in rugby and to constitute referee abuse. The uniform understanding of physical abuses led the coaches to focus, primarily, on other behaviors that may not be as easily categorized as abuse.

When considering referee abuse, the coaches noted that they wanted the ability to be competitive and to address their own disagreement with calls without having to worry that it would be misconstrued as abuse. HS pondered:

I can see how if you give a referee a wider latitude how me just arguing a call in a competitive manner could be construed as referee abuse and that's not something that I would say would be referee abuse, but on the same token, like, it's such a fine line between what would be acceptable and sportsman, if you will, and what would not be acceptable. It's just so tricky so you know, like, I'm sorry to hedge right in the middle of this answer. But if it's such a fine line between when you're trying to help your team and you're being competitive about it and when you're just being a jerk and yelling at a referee.

MX compounded HS's perception that arguing calls or yelling from the sidelines in and

of itself was not enough to be considered abuse. MX explained:

But referee abuse to me, you know, wouldn't be standing on the sidelines yelling out at the field - that isn't referee abuse. If I'm name-calling, if I go up and get in a referee's personal space and I'm being abusive meaning I'm calling him names or being personal about it. Or if I throw a punch or one of my player's throws a punch and it hits the referee. Or if we pull him into a ruck or tackle him or on purpose run him over, that's what I'm looking at referee abuse as. Me yelling at a referee and helping him ref the game or a parent in the grandstand, I don't consider that referee abuse. 
While all coaches wanted the opportunity to provide feedback to the referee, the appropriate method to do so was not agreed upon. Many of the coaches agreed with MX and HS that yelling alone would not be considered referee abuse. On the other hand, some coaches felt that yelling at a referee crossed the line and should be considered abuse. According to PZ, "If you're making someone miserable while they're doing something, then whether we like it or not, that kind of goes into the realm of abusing someone, if you don't intend to do it or not-you really are." PZ was not the only coach who felt referee abuse included yelling about a call. KU added, "Even if it's meant in humor, any verbal condemnation of a referee is the start of abuse." The differences of opinion regarding the categorization of yelling at the referee did, ultimately, lead to a consensus of abuse indicators among the coaches interviewed.

In attempting to define abuse, the coaches perceived specific indicators that would help one determine if abuse was occurring. For FQ, these indicators included the verbal expression of "anything vulgar or anything about someone's mother or any comments about family members." In this example, FQ denounced vulgarity and personally charged comments as abuse behaviors. Though the difference between yelling and yelling a personal comment may seem subtle, OY's perception seems to clarify that "saying a call is horse shit is one thing; calling the ref a piece of shit is different." When stated in these terms, it appears that while the coaches want some flexibility in voicing their opinions, most are aware when their behaviors have gone too far. "You can't be threatening, you can't be homophobic, you can't be sexist, you can't be racist, but outside of that . . banter, it's part and parcel of sport as long as it's done in good humor," says KU. 
GR expressed similar commentary:

I would say going above and beyond shouting at the referee-if you make it personal, name-calling or obscenities. Certainly, if you get in the referee's personal space, particularly in an aggressive posture where you're blocking their path or you're in an aggressive or intimidating stance. I think what was that old definition of pornography? I can't define it, but I know it when I see it.

There seems to be concurrence among 11 of the 15 rugby coaches interviewed that vulgarity and personal comments are an indicator of referee abuse. And, as seen above in the presented data, GR extended the definition of abuse to include physical behaviors such as entering the referee's personal space or blocking the path. GR highlighted the idea that these physical behaviors are meant to be intimidating. Thus, intimidation appears to be another indicator of abuse for nine rugby coaches. PZ explained it this way:

Anything that I think that makes a referee feel uncomfortable ranging all the way up to intimidation. I'd say as soon as you start to get into sort of the uncomfortable feeling for a referee, I think we're starting to border on that intimidation. You know, once they feel intimidated I think that's extreme. But I think you can be guilty of abuse if you put the referee in a situation where they feel uncomfortable and can't do the job they're supposed to be doing.

PZ's reflection underlined intimidation as another factor that coaches perceive may indicate referee abuse. A few coaches also suggested that repetition or tone may be indicators of referee abuse but also noted that these were not markers that could easily be included in a definition or clearly understood by everyone. Thus, the coaches tended to focus on vulgarity, personal comments, intimidation, and repetition as four indicators that could be utilized to define the differences between abusive and non-abusive behaviors.

Many of the coaches also expressed that they alone could not determine what might be considered referee abuse, suggesting that in some instances, the referee may be more or less sensitive to negative behaviors. 
GR explained:

I think that when a referee has informed you that they've heard enough from you and if you continue then I think that now you were treading on that line or possibly stepping over that line. I think once the referee has had to warn you about speaking to them it is incumbent upon you as a coach, you're responsible now at that point, to cease continuing in that vein. I don't know if this is going way off base, but you know I would say the same thing when you're approaching somebody on a social level. You know if you're making an overture towards a person and they let you know thank you but no thank you. No more. And if you continue you've crossed the line and if you say OK, message received, and you leave them alone, then I think, well, you found out where the line was-they drew it. Now it's your responsibility not to go further.

LV mirrored GR's sentiment that perhaps the referee should be responsible for defining what is and what is not considered an abusive behavior. LV suggested:

For instance, we were playing a game this year and we had a very vocal ref and we go into it with him. And when I say we, I mean my fellow coaches and I, not me specifically, have gotten into some arguments and even abusive type dialogue. Specifically, one of my fellow coaches and him. And he was the touch judge and my fellow coach was all over him as a touch judge and he told him if he doesn't shut up he's going to send him up into the stands. So, obviously he felt that the abuse had gone too far, and he shot back a little abuse himself, but to me that's a clear line. Like, hey, the line's drawn don't go any further. So, unless the ref gives some type of reaction how are you supposed to know?

Like GR and LV, HS noted that it is often difficult to know what other people are

thinking or to know when they may feel they are being abused. HS explains:

It's almost the golden rule. If you are the referee and somebody was yelling at you like that would you think it was referee abuse? Again, it's all personal to me because like what I might put up with would be different than what you would put up with as a referee, so it almost has to have a flexible line based on who the referee is just like any other rule or any other law out there, it's kind of based on how the referee wants to call it.

HS highlighted the idea that referee abuse should be defined based upon the biblical tradition of the golden rule: do unto others as you would have them do unto you. Thus, one's understanding of referee abuse must be informed not only by your own perceptions of abuse but also by the referee's perceptions of abuse. LV felt that referees had "kind of 
set [themselves] into a trap there by saying it's not up to us, it's up to the individual being abused." Determining which parties should be responsible for defining abuse or creating a definition of referee abuse that addresses both the perceptions of referees and the perceptions of coaches proved difficult. KU noted, "I suppose part of the difficulty is when that line starts to get blurry, right? It's like, then, from a coach's perspective, what you do you see and also with some referees [in the same instance they feel that] they're getting too much stick." Throughout the study, the coaches were cognizant that variable understandings of abuse were important in creating a universally operationalized definition of referee abuse in rugby.

As seen above, the coaches perceived physical contact to be easily defined as referee abuse. They also suggested four additional indicators of referee abuse: vulgarity, personal commentary, intimidation, and repetition. Finally, the coaches suggested that multiple perspectives of abuse, including that of both the coach and the referee, should be considered when defining referee abuse. Ultimately, said JA, "There has to be a standard, a baseline standard. This is acceptable, and this is not."

\section{Occurrences of Referee Abuse}

Despite the coaches' insistence that referee abuse had no place in the sport of rugby, all 15 of them indicated that they had witnessed referee abuse in their tenures as coaches. The abuses witnessed came from coaches, players, and spectators. To ensure that all of the coaches were considering the same definition of referee abuse, the following definition was verbally provided to all participants:

A verbal statement or physical act which implies or threatens physical harm to a referee or a referee's property or equipment. Abuse includes, but is not limited to, the following acts committed [against] a referee: using foul or abusive language 
toward a referee; spewing any beverage on a referee's personal property; or verbally threatening a referee. (Chiafullo, 1998, p. 202)

Following this definition, MX recounted one particularly egregious instance of referee abuse during a club rugby competition:

I saw referees get punched in the face. I saw all sorts of crazy stuff. We actually had to stop a couple games with shotguns because that day turned out they were abusing the refs and then we stood up for the referee and the fans, 150 fans, ran onto the field. I mean you know we had to shoot rifles into the air to stop the games.

MX's retelling of this story made it clear that physical violence perpetrated against a referee was unacceptable and that as coaches, they were willing to stand up for referees in these instances. The 15 coaches involved in this study suggested that though they had often heard stories of physical abuse occurring in American rugby, it was a rare occurrence. Only seven of the coaches reported that they had witnessed physical abuse. HS explained:

I literally had to warn a couple of people this weekend as an assistant referee. I see a lot of verbal, I see a lot of just you're an idiot, you're dumb, that's the wrong call. I see a lot of people doing the-where you throw your hands up and you're just like hurrhh [sic] and you make a loud noise all that stuff. Even that, I would consider abuse because if you look at our game internationally no one does that. You don't even do that. I don't see very much physical abuse, I've never seen anything thrown at a referee or you know someone squirting water at a referee. I've never seen anything on the field where the referee was targeted but I've heard about things happening. Definitely heard about referees and line judges being assaulted, middle of the match, end of the match, being intimidated, but I've never encountered that myself.

As mentioned here, there was consensus among the 15 coaches that the primary type of referee abuse that coaches encountered in rugby was verbal in nature. PZ noted, "Most of the stuff I've seen or experienced would absolutely $100 \%$ be verbal, you know swearing just calling them all sorts of ridiculous names and that type of stuff." Instances of verbal abuse appear to range from intense questioning of the referee to consistent vulgar attacks 
and threats. CN explained the type of verbal abuse that he has seen as a coach included "direct poisonously verbal attacks on referees-with plenty of explicative type of things." PZ extended upon the frequency in which vulgarity was used as a means to verbally abuse the referee:

You know I've been on a sideline where the opposing coach has used language that I probably wouldn't use outside of my close friends on a night out and it was relentless. You know, we're not talking about one or two instances in a game. We're literally talking about from the first minute to the 80th minute. Where you could tell everyone feels uncomfortable about it. Know you feel uncomfortable and I'm not even talking about just uncomfortable for the referee. You feel uncomfortable with the situation as a whole it's not a good environment.... Unfortunate, but consistent and pretty relentless-never spilled out to anything physical, never that I'm aware of spilled onto anything directly threatening harm or anything like that. But the language was, to your first point is the definition, the language was at best foul at worst something more than that.

As the coaches depicted their experiences, they continually explained that while physical abuse occurred infrequently, verbal abuse was commonplace. The verbal abuse that the Division I-A coaches had witnessed does not appear to discriminate and was seen across all sectors of rugby. MX noted, "I've seen referee abuse at the Division I-A level. I hear people yelling on the sideline." CN agreed with MX, stating, "I've seen it at pretty much every level." Though referee abuse appeared to be pervasive across the sport of rugby, most of the coaches interviewed found it to be more common at lower levels than at higher levels. Coaches defined the lower levels of rugby to be youth rugby or less competitive leagues. When referring to the higher levels of rugby, they seemed to be speaking of elite high school programs, collegiate rugby, club rugby, and professional rugby. Coaches referenced that at the lower level, they often encountered coaches and parents with little exposure to rugby culture. As a result, they brought their behaviors from other sports - including their proclivity to abuse a referee. 
According to PZ:

I have seen it more at the lower levels of high school—at high school programs. So, the better high school programs, DI programs, not seeing it. But the lower level programs where perhaps the sport is coming from a different culture, not necessarily race culture, but maybe not as long and deep in rugby culture-I see it more often.

Having not been exposed to the rugby culture outside of the United States, many coaches, parents, and players had been exposed only to an American sporting culture. In the American sporting culture, the coaches perceived that even at the lower levels of sport, parents were abusing the referees. AL explained:

I mean it's been awhile, but I umpired little league and coached baseball. But I can remember as an ump in little league baseball kicking parents out of the area because of their abuse. And I don't see that that has changed.

According to AL and many of the coaches, referee abuse seemed to be normalized and accepted in youth sport, which creates a sporting environment rife with abuses. DO explained the parental impact on abuses at the lower level noting, "I think the high school level is much worse than the collegiate level. I think a lot of that has to do with parents. You see more parents at games and parents want to protect their kids." By the time players, coaches, and spectators get to the elite college level, the coaches perceived that much of the referee abuse had subsided. LV explained:

Kind of like anything else, everywhere has its different social rules, norms. So, in a classroom you have to treat a teacher a certain way, and maybe you're a complete asshole outside of the classroom, but in the classroom if you behave badly, you'll get an F, so maybe you behave better. So just the same way you have to learn in a classroom to behave and you're kind of conditioned to do so throughout life, and you have ups and downs and hopefully figure it out by the time you get to college. It's the same with rugby. Maybe at the high school level it was allowed, or you had coaches that did it, we get kids from all different types of backgrounds, but when you get to the collegiate level, at least usually in our program, and you're competing at a high level and games can come down to a call here and there I think you're more conscious that the referee is important. 
Generally, the coaches interviewed agreed that as the level of rugby increased, so did the knowledge of the game and culture. As a result, there was more respect for the sport and for the referee, and thus, the instances of referee abuse decreased. While most coaches agreed that referee abuse was more prevalent at lower levels of competition, some coaches did note that these abusive behaviors had begun to creep into more elite levels of play. NW expressed how referee abuse was emerging at the professional level:

I have definitely seen there has been an increase in referee abuse even at the elite level. It's, obviously, for me growing up in England seeing soccer referees getting 20 guys in the referee's face, I've just never had any respect for that. And you're just starting to see it slowly creep in now.

As the stakes of elite competition are increased, two of the coaches suggested that the propensity for abuses may increase as well. In some instances, coaches' employment depends on the success of their rugby program; as a result, disagreements with the referee can seem that much more important. So while the majority of coaches found an abundance of referee abuse at the lower levels, others noted that elite competitions also saw their fair share of referee abuse.

Despite the existence of referee abuse in rugby, 13 of the coaches believed that the instances of referee abuse in rugby paled in comparison to other sports. AL suggested:

Rugby still stands significantly apart from the other sports cultures. You're going to find some different ones in there. I mean I think if you look at I'm trying to think of some of the ones that you know that I've seen you know let them have the respect- tennis is obviously one that has an ultimate respect for them for the linesmen, volleyball is pretty good for respect, but I think across the board rugby is still far above par from the rest of them.

Based on the perceptions of the majority of the coaches interviewed, less referee abuse existed in rugby than in other sports. There were, however, other sports that the coaches 
believed follow a similar tradition of respecting the referee. AL noted that tennis and volleyball also seem to follow that tradition, and other coaches mentioned golf and sailing as sports with less referee abuse than more mainstream sports.

While rugby may stand out among its sporting counterparts in championing respect for the referee, when comparing rugby in the United States to rugby in other nations, American rugby falls short.

I think it's a little worse here. I don't think it's football, I don't think it's football that drives the referee abuse. I think it's more basketball. And I think that's pretty much on display right now if you're watching the drama that's happened between players and referees in the NBA. But I think it's more prevalent here. (GR)

The notion that rugby referee abuse was more common in America than in other countries seemed to ring true for a number of coaches. Nearly all have had the opportunity to play, coach, or travel overseas with their rugby clubs. As a result, they have been exposed to rugby in both American and non-American settings. For MX, "the five years that I coached in New Zealand ... I never saw referee abuse. I never saw anyone yelling at the referee. It wasn't until I came back to America where I saw it." CN echoed this sentiment, expressing that referee abuse was "way more prevalent in this country than it was in [my home country]." These seven coaches related the prevalence of referee abuse in the United States to a lack of understanding of the rugby culture and/or the behaviors modeled by other sports in the American sporting culture. The perceived impact of culture will be discussed in a subsequent section where the factors that influenced coaches' perceptions of referee abuse are presented.

When considering the occurrences of referee abuse, the coaches understood referee abuse to exist within all levels of rugby, particularly at lower levels of the game. Of the abuse that they have witnessed, the most common type of was verbal, and while 
they have heard stories of physical abuse, personal experiences with physical abuse were limited. While the coaches acknowledged the existence of referee abuse in rugby, they felt that instances of referee abuse were more prevalent in American rugby than other rugby-playing nations. Beyond this, they believed referee abuse to be less prevalent in rugby than other American sports. The discussion of abuse occurrences led to dialogue regarding the potential negative outcomes resulting from referee abuse.

\section{Outcomes of Referee Abuse}

When discussing referee abuse, the 15 Division I-A coaches expressed their awareness of their sporting surroundings and were mindful of the impact of referee abuse within rugby. The coaches noted that referee abuse seems to impact rugby in three ways: (1) individual outcomes, (2) match outcomes, and (3) outcomes for the sport. HS explained:

I guess it goes without saying that any time you're abusive to anyone you know it certainly has a negative consequence that resonates probably far beyond the playing field and particularly when you have abuse of a match official somebody who is the authority in the match. I think the message that you send to everyone, players and spectators, youth, I think that message that you send out certainly then has an impact in the future, on other games, because people start to see that as permission as to how they can behave or act. So, I think that that means that we all have a vested interest to make sure that that doesn't happen.

According to eight of the coaches, the negative consequences of abuse begin with the impact on the individual referee. GR expanded upon the notion of personal impact of referee abuse: 'If you say referee, you're just horrible, you don't know what you're doing. That's affecting someone's mental well-being." The impact of referee abuse on one's mental well-being “can affect performance," said LV. "It's a high-pressure job and somebody near you being abusive and you're kind of being placed in an unsafe work environment would definitely impact their job and how they feel when they're doing it." 
Based upon the recollection of the coaches, referee abuse could, and they believe does, have a negative impact on referees personally, exposing them to unsafe work environments and undermining their mental well-being.

As referee abuse impacts the individual referee, the coaches believed there was a knock-on effect. OY explained, 'It's one of two things. The ref is intimidated and can't call good game, or the ref becomes vindictive, we're all humans." Therefore, referee abuse could impact the referee's ability to make appropriate calls or may even incite a bias in the referee as the referee becomes defensive or vindictive. In either instance, the referee was believed to be unable to perform his job to the best of his ability, and, as a result, the outcome of the match may have been compromised.

Referee abuse not only has the potential to undermine the referee and compromise the outcome of the match, but it may also impact the referee's continuation in his or her role. NW noted:

Some referees if they have a bad game where they're just constantly getting abuse it could potentially deter them away from ever refereeing a game again. And, obviously, in America there aren't a lot of referees, so we want to try to keep the ones we've got in the game and try and get more people into it. But, you're not going to be getting more referees if they know they're just going to be getting abuse. Same thing if you're refereeing and you have two to three weeks in a row of just getting abuse, you're like what am I doing this for.

As NW explained, referee abuse is a potential deterrent to both current and future referees. GR added:

Let's start with one-just the referee himself or herself. I don't think that anybody would stand for being treated that way and certainly wouldn't want to return to an environment that treats them in that regard. So, I think the first thing, the first immediate reaction is that we lose good people who are absolutely necessary for the function and participation of our sport. 
According to GR and NW, abuse behaviors could create an environment that would not be conducive to the participation of referees and would make them continually reconsider if they should continue to participate. HS suggested, "Who wants to referee a sport if you're going to get beat up?" CN mirrored that sentiment:

I think it undermines confidence and trust in the whole system and the whole setup and the whole idea of referees to a certain extent and I'm certain it would affect the psyche or the mindset of the referee, not just about necessarily how they referee, but whether they really want to continue refereeing.

Referee abuse was perceived to be off-putting for both current and potential referees. $\mathrm{CN}$ continued:

I see it actually being a real problem for us when trying to get referees. As much as people bemoan referees and all that stuff, we can't really play a game of rugby without referees. So, I know because I'm a level 1 referee and [my referee society] is emailing all of the time trying to find referees. So, I know it's a really difficult thing to get people into. So, I'm sure the abuse factor is definitely one of the elements that prevents people from taking up the whistle anywhere.

According to 11 of the 15 coaches, referee abuse was likely to have a negative impact on referee recruitment or retention. The coaches suggested that referees do not want to operate in a workplace where they feel unsafe or are treated poorly and thus may hesitate to continue refereeing under such abusive circumstances. The coaches interviewed suggested that of the negative outcomes associated with referee abuse, reduced recruitment and retention of officials may be the principal concern. OY explained:

The biggest impact [is] it stops more referees from joining and that's the biggest concern. In my hierarchy of what is needed in rugby is a plot of land, a ball, and players. Without those things we don't have the sport. Coming in right behind those - we need referees, and we need good referees. ... So, I think referee abuse is harming that portion of it. 
As OY noted, referees are an organizational requirement to enact a successful rugby match. Continued referee abuse was perceived to incite referees to question their participation in rugby and, at times, leave the profession.

Beyond the issues of recruitment and retention, which the coaches believed to be enflamed by referee abuse, three of the coaches also suggested that referee abuse detracts from the value of rugby as a sporting product. JA explained:

If I'm a corporate sponsor, if I'm coming from Ford, and I go to a ballgame. And you just get ... it's like a bunch of hooligans screaming at the refs. I don't know, I think it cheapens the totality of the greatness of the sport and of the product itself. ... So, I think it just really hurts the product and I think it hurts the growth and development.

In this way referee abuse has the potential to set a bad precedent for the sport, exposing a weakness to potential investors and reducing the value of the sporting product.

You never know who is there! The way I tell it to the guys is everyone there is a potential sponsor, supporter, or financial backer [of] the sport. You want to abuse this guy? People screaming and yelling? You want to be a part of that? Is that that something I want to be a part of? Probably not. (JA)

As JA explained, the impact of referee abuse can reach far beyond the pitch, suggesting that every spectator may be a potential investor. Allowing referee abuse to continue may potentially decrease the marketability of rugby as a product: "It's a crummy environment; it just casts a shadow on the program and the event itself," explained JA.

The potential negative impacts of referee abuse are well-documented by the Division I-A rugby coaches. The coaches highlight the trickle-down effect of referee abuse on the game of rugby, beginning with the impact on the referee's personal wellbeing. As the referee is personally impacted by abuse, they are perceived to possibly become biased or have the potential to err in their decision-making, ultimately expressing a larger impact on the game. Beyond individual and match-level outcomes, referee abuse 
was believed to detract from an organization's ability to recruit and retain referees and to secure sponsorship resources. The coaches in this study perceived the outcomes of referee abuse to be incredibly negative, impacting rugby at many levels.

The exploration of Division I-A rugby coaches' perceptions of referee abuse highlighted three emergent themes: (1) difficulty in defining referee abuse; (2) the occurrences of referee abuse in rugby; and (3) the perceived outcomes of referee abuse. The coaches agreed that referee abuse did not belong in the sport of rugby and that physical referee abuse was relatively simple to define. Defining verbal abuse proved to be more challenging. To do so, the coaches identified a number of behaviors that suggested a line had been crossed and that verbal abuse was present. Abuse indicators included foul language, repetition, intimidation, and personal comments. The coaches also suggested that the coach's perspective alone was not enough to create a definition of referee abuse but that the input of other sporting stakeholders should be solicited. Both the coaches' ability to suggest indicators of abuse as well as their personal experiences suggest that referee abuse was ever present. According to the coaches, referee abuse occurred most commonly at the youth and high school levels. Though referee abuse was less pervasive in rugby than in other sporting contexts, witnessing referee abuse in rugby in the United States was more likely than in other rugby-playing countries. Finally, the coaches indicated the detrimental outcomes of referee abuse on the sport of rugby, the match, and the individual referee.

\section{Factors Influencing Coaches' Perceptions of Referee Abuse}

Answering the first research question in the previous section created a context from which to explore the factors influencing coaches' perceptions of referee abuse (Q2). 
Five themes emerged to describe the factors influencing the coaches' perceptions and, subsequently, their likelihood to engage in referee abuse. The coaches' perceptions of abuse were influenced by (1) the personal characteristics/philosophy of the coach, (2) the coach's relationship with referees, (3) social influences, (4) organizational role expectations, and (5) culture. Each of these emergent themes is distinct yet related to the others, as the factors influencing the coach do not exist independently.

\section{Personal Characteristics and Philosophy of the Coach}

When considering what may influence a coach's perception of referee abuse, the coaches suggested that we must begin at the source. Innate characteristics, selfawareness, and accepted philosophies of a coach impact their perceptions of referee abuse and their likelihood to engage in abuse behaviors. According to NW:

Each person is completely unique and individual. Some people are more likely to try and have that abuse at the referee and if a referee starts giving them yellow cards and stuff like that, usually, it's going to antagonize them even more. Whereas others, if someone gets a yellow card incorrectly, they may just accept the decision. Again, it's all on the innate personality of the individual if they sort of take it or they want to fight back.

In this example, NW explained how one's personality characteristics may impact his or her predisposition to engage in referee abuse. Some individuals may be able to internalize their feelings and accept decisions made by the referee, while others become more vocal and express their discontent when mistakes occur. The basis of these alternatives is associated with one's innate personality. NW continued:

I mean obviously you've got different personalities out there across the entire team and in the stands, as well. And some people enjoy that confrontation and that sort of argumentative state. Myself, I've never been, I've got no time for it myself. Obviously, part of your personality-if you like to be vocal about your thoughts and stuff like that-so if your thought is that was a terrible call by the referee, you're going to outlay that to the people around you. 
NW continued to highlight how differing personalities may lead an individual toward or away from confrontation. HS explained:

Maybe it's a little bit of personal-what's even the right word-if the coach is a hothead in real life, if he or she is a very reactionary person, that carries over into rugby, that carries over into all aspects of someone's life.

According to the coaches, stepping onto the pitch does not allow the coach to disassociate with their everyday personality traits. These traits exist regardless of the context and thus impact one's perception of abuse and the likelihood to abuse the referee. One specific personality trait that 11 coaches believed to impact referee abuse was ego. Ego from both the coaches and the referees seemed to influence abuse perceptions. GR explained:

I mean one of the things that's commonly said is the referee is the sole judge of law. Right? Or as we would say, the referee is God on the field. Whatever the referee says goes no matter what. So, what I told that group of referees was if you're God and if you think about god without getting too religious, god is all knowing, all powerful, but also merciful. God is kind, God is loving. And unfortunately, we deal with a fair number of referees where that power has gone to their head and they're just going to referee with a vengeance.

While many referees are perceived to be seeking power, a number of coaches highlighted that a coach's ego also influences his or her abuse behaviors. Coaches want to be perceived as being correct and having all of the information. For JA, abuse stems from the "egos. Know it alls. In rugby you've got a lot of know it alls." OY extended upon this notion and suggested:

This is my opinion, they feel that their working knowledge is as good as anyone's. Then you deal with a lot of alpha males in sport, it's easy to make up for your shortcomings by looking at the shortcomings of others. That's not a good thing, that's just what I see ... 99\% of the time it's ego on both sides.

Ego seems to come into play from both the referee and the coach. In either instance, the personality trait of the individual is seen to influence a coach's perception of referee 
abuse. According to DB, “They're all intertwined. You might have a shocking referee full stop, you may have a shocking coach full stop. At the end of the day both guys think they're right." Based upon the coaches' discussions, the desire to be right seemed to fuel both the coach's and referee's egotistical behavior, potentially leading to frustration and abuses.

Despite individual innate personality traits, eight of the coaches believed that professional development and a growth mindset could help coaches be more cognizant of their potential for abuse behaviors. For DB, it took a reflective approach to change the perception of referee abuse. DB recounted:

Just at the end of the day, look, I just knew myself. I just wasn't the best person that I could have been. Abusing the refs or being too hard on some of the players, you just come home when the dust has settled, and hindsight is 20/20 and you just know I shouldn't have done that.

Some of the coaches expressed that self-reflection had enabled them to move beyond their innate personality characteristics and make decisions rather than react. EP remembered early in their coaching career when they were known as a yeller and a referee abuser. EP explained:

And I'm not throwing stones in a glass house, I had to go do that in 2009. As a player transitioning to a coach, and all the life stuff that was piling on, I had to go and do that. It was interesting. It's interesting that I'm saying that because at one point I was a frustrated young adult. I was becoming a HS coach, I was becoming a collegiate women's coach. Fortunately, I had a partner at the time who at one of my outbursts - which was a Del Taco bag and a soda cup in a tree- she just looked at me and goes, "does that make you feel better?" I said, "nah, it doesn't." And she said, "well, maybe we should start working on some things." And I was like okay, you know and then what is it 2018, nine years later and I'm self-aware, I'm much more self-aware than I was then.

For many coaches, the transition from player to coach was difficult, described as a time when they were forced to evaluate their own values and the philosophies that guided their 
behaviors. When transitioning into a coaching role, the coaches noted that it was often difficult for them to step back and realize that they are no longer on the field and that their behavior influences numerous other individuals. PZ recalled:

When I was younger, I was definitely a firebrand and I would sit with refs afterwards and talk to them and try to figure out what it was that would make me do that. And it's been awhile, probably the last eight or nine years where that's changed.

These coaches perceived that reflecting on their behaviors allowed them to find clarity and, at times, reconsider their previous actions. LV noted that their abuse behaviors decreased overtime as a result of self-reflection:

I mean, my own kind of coaching development, maturity, and honestly seeing others. I see others and they're bitching about every call and I think they look like idiots. So, they may be wrong this time, but there's plenty of times when I didn't get the call and I was vocalizing it and I looked like an idiot.

Being able to reflect upon their own actions and the actions of others has allowed LV and others to consider the impact of their behaviors. OY explained, "One of the reasons I really started to temper it was because I would see videos of me yelling all game, whether it was abuse or not. That's all I was doing, how was I helping anything?" For many, it took a period of self-reflection to stop excusing their own behaviors and change their perceptions of abuse and their likelihood to engage in abuse behaviors.

Both the innate characteristics of an individual and his or her willingness to selfreflect are personal characteristics that the coaches believed influenced their role in referee abuse. Further, for 13 of the coaches, their coaching philosophy also served as a factor that influenced their actions and perceptions.

Your coaching philosophy is probably a direct reflection of how you approach most things in life, and I think that some people have a mindset of continued growth, and learning, and cooperation and others are more hard-headed and so that leads into how they treat referees. (LV) 
Here, LV explains the convergence of a coach's innate personal characteristics, his or her openness to personal development, and his or her coaching philosophy. A slight majority of coaches $(53 \%)$ suggested that within their coaching philosophy, a focus on outcome, or wins, led them to be more aggressive with the referee.

When I focused on outcome, I was a much angrier person and the frustration trickled into what we talked about before the fairness of calls, what I was deeming unfairness to my players got me to be frustrated, I was also not mature enough at the time to go and be self-aware, but I was also worried about the win. So, winning caused me to have more frustration. When I started to focus, and I was enlightened to the way of my athletes' performance is first and foremost, and my performance as a coach is first and foremost, and that if I get that piece right, if I get my performance right, my players get their performance right, then winning is an outcome of good performance. Then I never worried about winning and all I did was focus on my players, that's when I changed. (EP)

With a focus on strong performance rather than outcome alone, EP believed they were able to impact the likelihood that they would abuse a referee. The focus on performance required EP to take responsibility for personal actions and for the training that had been provided to the players. Good training would result in good outcomes. In this way, the referee became a non-factor in the outcome of the game, and EP was able to decrease the personal tendency to abuse the referee. As EP reflected on the relationship between the referee and the team's win/loss record, EP acknowledged:

A win or an outcome is the result of good performance. So, we acknowledge that we don't have control over the referees, but we need to adjust to the referee just like it's a condition in the weather. The referee's interpretation of law could be considered an adjustment just like the rain, the snow, sunshine, a narrow pitch, a wide pitch, a short pitch, same thing for us.

Other coaches' perceptions of referee abuse were also impacted by their philosophies. FQ noted:

Nobody talks to referee except the captain or myself. That's it. If there's going to be anybody that has an issue with it leave it up to the to the person that you were 
elected to stick up for you-your captain — and me whose job it is to do that. So, I guess it's teaching them like teaching them like hey this is [University] way.

We're not going to do that here. I don't care how you did it before, but here we're not going to do it. It's usually just like chirps like, "sir what are you doing that's not a penalty" or just dumb things like that.

In this way, FQ utilized a coaching frame to ensure that the players were not abusing the referee. Allowing conversations with the referee to occur through two individuals, the coach or captain helped negate extraneous referee abuse or chatter. Thus, the coach's philosophy impacted not only the behavior toward referees but also helped mitigate abuse behaviors from players. AL's perceptions paralleled FQ's:

I think number one is that coaches not accept it from their players. I know I've had young players that I've taken off the field immediately and played short because they were young, they were enthusiastic they hadn't absorbed the culture yet. So, if you if you take immediate action whenever you see it against a referee, that's number one. Number two is you don't accept anything less than, you know, a staunch character even in practices.

Again, AL's comment highlighted how the coach's philosophy and execution of said philosophy not only impacted the way that the coach may behave but also impacted the team's perception of referee abuse and subsequent abuse behaviors. If referee abuse is unacceptable according to a coach's philosophy, both their behaviors and the behaviors of their players seemed to mirror this philosophy. KU suggested:

It's got to be a constant message of what's acceptable as a team. You know and it's a constant message, constant message, constant message. Only person that talks to the referee is the captain. And he's going to approach the referee and he's going to talk to him in this manner and it's constant. Respect the opponent. Respect the game. Respect yourself. Respect the referee. So, it's just it's constant at practice, it's constant game day, so you've got to have consistency.

Consistently talking about it. And then setting expectations and when those expectations are not in place then punishing people internally, letting the group know, making examples of people, that that behavior will not be accepted in our program and then from that point on it's a growth mind-set. 
A philosophy of respect impacted not only the coach's perceptions and behaviors but also those of the players. Coaches who were constantly discussing respect for all participants in the game, including referees, hold themselves to a high standard. DO noted:

We wouldn't do that to each other so why would we do it to someone else? And we have a similar zero tolerance policy internally for anything that just might not fit in terms of being a good human being, and referee abuse obviously doesn't fit in that.

DO's coaching philosophy, like those of the majority of coaches interviewed, suggested that all participants in the game of rugby should be respected. DO highlighted that abuse among teammates would be considered unacceptable, as would coach abuse of players; thus, the same expectation holds true for coaches and players regarding referees. The coaches believed that one's coaching philosophy may impact perceptions of abuse and abuse behaviors.

There are numerous factors that may impact coaches' perception and actions related to referee abuse, including their personal characteristics and philosophies. As discussed, the innate characteristics of individuals, their openness to self-reflection, and their accepted coaching philosophies converge to form their perception of referee abuse and their subsequent likelihood to engage in abuse behaviors. The personal characteristics and philosophies of coaches not only impacted them but also appeared to impact their players. Because the players also adopt the philosophies of a coach, the influence of a coach's personal characteristics and philosophies on referee abuse is exponentially greater than factors that may influence the coach alone. In addition to the impact of personal characteristics and philosophies on one's perception of referee abuse, the coaches noted that relationships with the referee also play an important role. 


\section{Relationships with Referees}

In this study, coaches noted that having a strong relationship with referees humanized the individual and served as a deterrent from abuse behaviors. Few coaches claimed to have strong relationships with the referees officiating their competitions, but all of the coaches noted that relationships with referees may impact the way they engage with that individual.

Same way you would see benefits from building a relationship between your players and your coach type of thing, you know. Just like we're all in this together-it's not really about the referees or the coaches, it's about the players. But, we are there to help the players to enjoy the game and to help them be successful in the game as much as we possibly can, so I think that that definitely helps and the more you know someone the less likely you are to engage in abuse, to be perfectly honest. (CN)

$\mathrm{CN}$ noted that as relationships develop, one is less likely to engage in abuse. "I know so many people and I know the referees personally, I'm not going to risk that personal friendship by yelling about a knock on," said HS, who went on to explain that a personal friendship with referees would be prioritized over a call in the game. PZ felt similarly, reflecting on the advice that a friend (a referee) had given:

And now you know I have a couple of USA panel refs that are friends and one with them has coached with me in the past and their advice has always been how about you try to figure out something about [the referee] away from the game. I think once you get to realize that they do it for enjoyment just like you, you know maybe they didn't want to be a coach. Maybe they didn't want to be a player, or maybe this was what they decided was their way to contribute to the game. Once coaches understand that more, I think we start to look at the rest differently and see them as sort of the critical part of game day, and the critical part of, actually, coaches getting better because they tend to educate us.

Getting to know the referee on a personal level appeared to increase the perceived value of the referee to the overall sporting context. Coaches explained that they would be less likely to abuse a referee whom they saw as a friend and valued as part of the game. As 
coaches develop relationships with referees and begin to humanize the individual, these ideals were reportedly passed along to their players. GR provided a great example of how a relationship with a referee influenced GR's perceptions of referee abuse and helped prevent abuse behaviors:

We need to have more interaction off the field with coaches and referees because I think when you get to know each other and ... I've got an example for you. [Referee] did one of our matches, I wasn't particularly pleased with his performance, but I really like him, and I have a tremendous amount of respect for him. At half time, and you know I'm a little agitated, but he's a good guy and we've had - I don't want to say a great friendship, but I would like to say that we have a great amount of respect for each other. So, I walk out onto the field quietly and I say to him, [Jimmy], what's happening at the breakdowns, man? We're getting crushed. He starts kind of talking me through what he's thinking and what he's seeing and why he's calling what he's calling. I said well, okay, here's the penalty count - I just didn't think we're that bad. And he said well this is what your guys are doing. So, I was able to walk over and talk to my guys and say this is what he's seeing. And after the game I was able to walk over and talk to himbut I couldn't be mad at him. We had a disagreement in what we saw, but ultimately what he saw mattered most and my job was to help my guys adjust. I think because of the friendship, because of the respect, because [the referee's] a good guy, and also, I mean I love and respect the fact that anyone that's going to take time out of their own schedule to come out and blow the whistle for our boys to enjoy playing. There's got to be something that you automatically give just for that. You're giving of yourself, your time, so thank you. So, but I think the more that we can get our coaches and our referees to engage off the field the less likelihood that we're going to see the bad behavior on the field.

In this instance, GR's relationship with the referee allowed GR to have an open and honest conversation about the calls being made. As GR explained, once the referee's rationale for decisions was understood, GR's anger subsided. While GR may have been frustrated by or disagreed with the decisions, GR respected the relationship and the individual enough to maintain composure and help the players adjust to the referee's calls. Extending upon GR's belief that relationships help prevent poor behavior on the field, NV posited: 
I think in this situation it comes down to relationships. Once everyone realizes that we're all human beings, nobody is out here to screw anybody over, it humanizes the situation and it's a lot harder to have that negative undertone and tension. Which I think is great for refereeing, but at the same time you're not always going to know your referees, so I think it's tough.

The difficulty, as NZ mentioned, was that in most instances, the coaches do not know their referees or have the opportunity to develop a strong relationship with them.

Typically, referees and coaches have little time to interact outside of match day. As a result, FQ discussed the difficulty in building relationships with referees:

I do go out of my way on game days to make sure I talk to them as much as possible, like oh we have a trainer, we have water, we have this, we have that for you, just let me know. But that's the extent of my knowledge of them.

While there was consensus that relationships between the referees and coaches created positive outcomes in regard to reducing abuse behaviors and changing perceptions of abuse, coaches also noted that at present there were limited opportunities for relationship building. Those who had relationships with referees reported positive interactions and a decreased likelihood for referee abuse.

\section{Social Influencers}

Coaches acknowledged that they do not exist in a bubble; thus, the third factor that may influence referee abuse was their social environment. Twelve of the coaches found themselves to be influential figures within the rugby sphere, noting their responsibility as a role model for their players and programs. As a result, these coaches believed that it was their responsibility to exert a positive social influence on their players, colleagues, and fans. JA explained, 'It's the coach's responsibility $100 \%$. If the coach says I'm going to yell, 'rah, rah,' players will follow suit, 100\%." With such 
influence over their player's behaviors, coaches believe that they are accountable for any referee abuse that occurs within their program. OY agreed wholeheartedly:

It starts with the coaches and comes from the coach. Everything in our sport starts with the coach. The fans feed off the coaches, the players feed off the coaches, the coach can stop the players, the coach can stop the fans.

In this way, the coaches felt that referee abuse in rugby could be traced back to the views and behaviors of the coaches. NW explained:

I always think how your players act is a reflection on you as a coach. So, when I've had some of my players and people in the stands shouting abuse at a referee, I consider that reflective of me. And obviously I'm a big advocate for just accepting a decision and dealing with it, so when I see my players and supporters shouting abuse at a referee, I see that as a reflection of me, which doesn't do me any good.

Because player behavior tended to reflect the actions modeled by the coach, the coaches interviewed became obligated to mitigate their abuse behaviors. In this way, the coaches' perceptions of abuse or likelihood to abuse a referee were influenced by their players' likelihood to replicate their actions.

I just think in any sporting context the coach is in a leadership position. Especially, I coach young men-18-23-year olds - and they kind of are looking for guidance and example setting, so if a coach is abusing a referee it's obviously giving the impression that it's ok to do so to your players, as well as to the other coaches across from you. And parent groups as well, so I think a lot of people can take the lead from the coach in those situations. So, if they do it they'll lead to others doing it for sure. (LV)

As LV explained, the behavior of a coach has a domino effect. Coaches who abuse the referee give credence to the notion that referee abuse is acceptable and tolerated. Players, other coaches, and even spectators might then perceive that abuse is appropriate. According to PZ:

I'm responsible for not only my own behavior but all of the players, and our fans, and everything that happens at our home field. And I think it does-you do that and that perception carries with you. I think it sort of negatively impacts the rest 
of the division, or conference, or league, or whatever part you're in. Because if you allow it to happen in one setting you can almost guarantee that it'll happen in another.

Coaches believed they served as role models not only for their players but also for spectators.

Beyond the influence of the coach as a role model, the coaches also noted that peer influence could play a role in rationalizing abuse behaviors and in proliferating the perception that abuse is tolerated within the sport of rugby. DO found the herd mentality among players enabled abuse behaviors:

Friends' influence is huge. Social pressure is huge. If kids see somebody else doing it and then they've been told that it's ok, that just gives them that much more feeling of freedom, like oh, I can do that too. Or she got away with it, I can do that too. Social pressure is huge.

DO reported that peer influences created new acceptable standards of behavior, which both rationalized and supported referee abuse. PZ explained:

Historically it's been it's OK to yell at the refs. I guess it's been okay to do it. Parent one does it and it's like you've got dominoes falling. One does it, two does it, three does it, four does it. They'll think it's cool because nobody can stand tall and go hey you don't know the laws, right. Guy is having a tough time. The kids don't really know all the laws. Give this guy, give him an opportunity try to manage, talk to him after the game.

One parent or spectator engaging in referee abuse potentially made it acceptable for another to follow suit. PZ continued, "I think once you get a handful of people the confidence of doing stuff and saying stuff without getting in trouble gets higher and that's what I call the mob mentality." When discussing the ways peer influence impacted perceptions of referee abuse and abuse behaviors, coaches continually emphasized that behaviors proliferate only if there is no consequence. In this way, coaches acknowledged that their own behaviors must send the message that referee abuse is not to be tolerated. 
Coaches noted that it was their responsibility to engage both their players and spectators to curb abuses and hold them accountable to the standards of the program.

According to the coaches, role modeling and peer influence were social influencers impacting one's perceptions of referee abuse and likelihood to abuse a referee. The coaches prioritize their impact on other sporting stakeholders rather than focus on the social stimuli that influence their behaviors and perceptions. Despite this focus, it was also noted that a byproduct of the coaches' role modeling was a moderation in abuse behaviors. Coaches highlighted their tendency to moderate their potential abuse behaviors because they were aware that their players were watching. By having such an influential role on the behaviors of others, coaches are also, if inadvertently, influencing their own abuse behaviors. As a result, social influencers are seen to impact referee abuse perceptions for all sporting stakeholders, including the coach.

\section{Organizational Expectations}

In addition to personal characteristics, relationships, and social influencers, 14 of the 15 coaches noted that expectations created by their organization or institutions were a factor that influenced their abuse perceptions. According to the coaches, the stature of the Division I-A rugby competition created certain expectations of quality for the competition, including role expectations for the referee. In most instances, the coaches noted that the referees involved in this competition are not prepared to officiate such high-caliber matches. Thus, the coaches' expectations of a referee's role-to provide a safe and fair playing environment—was often left unfulfilled. DO noted:

I would say safety is probably the number one thing, things like high tackles or dangerous plays. Sometimes in my experience it can also be about fairness. The referee's not calling or is calling the game seemingly one sided or the referee seems to be not qualified enough to referee the level of this match. 
As expressed, when expectations of safety and fairness were unmet, the coaches

perceived a greater likelihood that referee abuse behaviors did exist. EP explained:

I've always coached to the letter of the law and I've coached my players to play to the letter of the law and not to engage in cheap banter or cheap shots or any of those things. So, any time I saw another team utilizing tactics that I saw as cheap, or foul or dirty and they went unpunished by the referee and my team was subsequently punished for retaliating for what they were getting. I think the retaliation law or the circumstance of retaliation in terms of cards in rugby is absolutely stupid. The person who gets punched and reacts because they're just a human and their fight or flight response kicks in-I think it's stupid not to punish both parties. Punish the aggressor and the reactionary or the aggressee or whatever you want to call it. Don't just punish one. You know, I've always felt that that was an unfair circumstance in the game. And when I was a young coach, I let that get to me, I let that frustrate me, because how could I coach like this and expect the values of the game and the laws of the game to be upheld and it's not.

Coaches expected that if they coach in accordance with the laws of the game, their team would also be officiated in accordance. Any deviation from this expectation brought up questions of fairness. When the referee was perceived to be unfair, by interpreting the laws of the game differently from the coach, the disparity led to coach frustration and oftentimes was believed to be a precursor to referee abuse. NW recounts an instance of bias encountered during a match:

When you're experiencing that sort of bias against you. Like in that one game I was asking the referee what do I need to do to get a penalty and he said 'I've got to start calling them.' I'm feeling a lot of hostility between us because he's not giving my team the penalties we supposedly deserve, which he's already admitted we deserve, but like I've said if a referee is poor to both teams, I've got no problem with that. It's when referee is considered inconsistent or biased against one or another that's when it's going to be a more hostile environment between the coach and referee. And obviously that biased and inconsistent refereeing is always going to be a cause for referee abuse.

As exemplified, 14 of the coaches recalled occasions where referee abuse had resulted from unfulfilled expectations and perceptions of injustice. 
HS explained one such scenario:

I think maybe the number one reason why someone assaults a referee is that the referee makes a bad call or maybe makes a call that they feel is bad if it's a close call and it just drives people over the edge. For instance, we had a high school game in Colorado a couple weeks ago where a referee made a call that the team did not agree with and the coach of that team, who is also a referee I might add, pulled his entire team off the field with time left and was like this is it, we're done, we're leaving, this is not safe. The referee wasn't wrong. The referee made the right call. The coach overreacted. So, it's probably a major cause for the referee abuse that happens.

OY summed up the coaches' sentiment concisely, stating, "It's just as simple. It's interpretation of the laws and as long as it's fair I understand it. If it's not fair and it puts people in the negative that's a problem." The coaches' expectations for referee fairness within their competition and within the USA Rugby sporting structure were a factor that influenced their perceptions of referee abuse and the likelihood to engage in abusive behaviors. Despite this belief, coaches also acknowledged that perceptions of fairness are incredibly subjective. "The fairness part is all subjective anyway. One coach will think it's fair and one coach won't and most of the time we're just being idiots when we're thinking about things that way," suggested PZ. So, while coaches do feel that there is an expectation of fairness within a game, they also acknowledge that this expectation may be difficult to fulfill.

At times, the subjectivity of fairness expectations was further muddied by the inclusion of a safety issue. For 12 coaches, it was paramount that referees not only officiate a match in a fair and consistent way but also protect the players in the game. To the coaches, a safe playing environment was a requirement for organized competition at the Division I-A level. LV and 11 of the other coaches believed that a referee's inability 
to maintain a safe environment could play a role in abuse perceptions and subsequent abusive behaviors. LV recounted:

I think when it comes out of me is when I think that something unjust is happening - so if someone is calling calls against your team that you don't see or don't agree with or a ref is missing a call. For instance, one of my players on the side of the scrum was getting punched in the face by Cal's flanker, so if I see something unjust like that and the refs not seeing it. I didn't yell anything abusive, but I can understand why a coach would.

In this instance, the perception of injustice resulted from a safety concern that went unpunished. In considering the referee's responsibility in the game, the coaches expected fairness and consistency of calls and that their players would be protected for the duration of the game. HS explained, "Safety is, like, above and beyond everything else. I think safety is always on everyone's mind. And the goal of any good coach or referee is to get everyone through the game safely." When safety expectations are not upheld by the referee, coaches often took it upon themselves to seek retribution. For FQ, safety is the one instance in which referee abuse may be justified.

I think the safety issue, I will never fault a coach especially if there's video evidence, which there's now more and more of, if they berate, verbalize, and abuse a referee if it protects those players. Especially if there's actual evidence to prove what the guy was doing was wrong and dangerous.

GR emphasized:

The safety issue is a problem, because as coaches we certainly have a responsibility - we have a legal responsibility and a moral responsibility for safety. But, also, that's your kid that you don't want to see hurt or abused or injured. And if a referee cannot keep that player safe, or all the players safe on the field, we have a real issue. Now, I don't, I haven't been in too many of those situations, but I have been in a few of them where it is unsafe, and I think you put all parties in a real difficult position when you have safety as an issue, especially if the referee is not aware. So that one's a tricky one. You know that's probably not shouting from the sideline, but it would be something that's probably not ... it might be a conference between the coaches and referees whether it be at halftime. But when you see unsafe action happening over and over again, you 
know as a coach you have to stand up for your players in this situation. So that one's a tough one.

Safety seemed to be one of the few issues where coaches collectively began to reconsider their zero-tolerance stance against referee abuse. Both coaches and referees are tasked with protecting the players throughout the course of a match, but the issue seems to go beyond mere responsibility. Coaches sought to protect their players because, as GR noted, "That's their kid." They are personally invested in the well-being of their players and thus do not want to see any of them succumb to injuries. When a referee jeopardized that, the coaches felt the innate desire to protect their players, which at times may have resulted in referee abuse. HS explained how this often played out in the context of a match:

So, I think that coaches are always concerned about the safety of players. So, when high tackles happen, when questionable legal you know brutality kind of plays happen, it is very easy to be like you don't care about my kids. I don't care about you.

In this way, the coaches' aforementioned concerns for the well-being of the referee were trumped by their responsibility to protect their players. The expectation of safety was the only circumstance in which coaches were willing to pardon referee abuse. PZ noted:

I will in no way shape or form try to deny that I will criticize a ref if I see that they are incapable of keeping players safe. My opinion is that the referee's number one job is not to give penalties and all that sort of stuff, it is to ensure that 46 players remain safe. And I'm not sure that we have a set of refs that have the full technical expertise to do that. There have been some notable and very public and fairly horrific examples of it, but I do think that what we're probably forgetting in our ref education is that number one priority is player safety. I would be less than pleased with the referee if I feel that the safety of the players is being compromised. The decisions and the technical aspects and all that stuff-I could care less what the decision is as long as they're consistent - but you know from a safety perspective that's got to be number one. And if that's not number one then there are definitely going to be clashes and conflicts between the refs and the coaches. 
The coaches in this study agreed that their expectation for maintaining a safe playing environment is the referee's primary charge. When referees do not fulfill this expectation, coaches are more likely to condone abuses. Collectively, the coaches expressed that their perceptions of referee abuse were influenced by the organizational expectations of fairness and safety.

\section{Culture}

In the previous sections, the Division I-A coaches identified personal characteristics, relationships, social influences, and organizational expectations as factors that influence their perceptions of referee abuse. The final factor that all 15 coaches believed influences referee abuse was culture. The term culture was used to discuss numerous facets of rugby and how they may interact with one's perception of referee abuse. Culture was referenced in regard to the rugby culture, the American sporting culture, and the soccer sporting culture. Each of these cultural derivatives were believed to influence perceptions of referee abuse in various ways. When discussing rugby culture, sometimes referred to as international rugby culture, there was consensus that rugby was meant to be a gentleman's game, with the sport prioritizing the values of rugby and seeking to uphold the ethos and spirit of the game. HS noted:

One of the things I feel like we as rugby players, if I can speak for us as a whole, that we pride ourselves on is the idea that we have this higher standard in sport that it's a gentleman's game. That whole thing. I think when you have people that are abusing referees you send the wrong message especially to young players that, again not trying to sound pompous here, but that we're like any other American sport where you know parents and players everybody yells at the referee.

Coaches or players who have grown up in a rugby culture or have played for a coach who introduced them to the rugby culture were believed to be less likely to accept or to engage in referee abuse as they sought to uphold the values of rugby. 
We're known, rugby all around the world is known, for setting the tone about respecting each other as players on the field - we shake hands and we drink beer together, and we eat together. No other sport in the world that that happens. We're also the only sport in the world that treats the referee with so much respect. So even in NZ, because rugby is their national sport and every kid that grows up wants to be an All Black, I think they treat the referees across the board better. (MX)

Twelve coaches noted that respect was the primary value that was associated with rugby culture- - "respect for their opponents, respect for the game, respect for themselves, certainly respect for the referee. I think that is probably one of the critical principles in rugby is that respect and sportsmanship that we show to everyone involved," explained GR. The value of respect epitomizes rugby culture and operationalized the meaning of gentleman in a "gentleman's game." When considering the way that rugby impacted one's likelihood to abuse a referee, HS reflected:

A big aspect of our sport, internationally, is the idea that for a long time in rugby, you had a referee, but the referee was literally only there if the two captains of the team couldn't decide on what should happen. The referee overruled both of them, you know. If they were like "Oh, that's a knock-on call, that's a knock on," the referee didn't make that call, the captains of the teams did, and that's kind of the gentlemanly aspect of the sport.

The rugby tradition of respecting the referee's decision without question has also become part of rugby culture. NW explained:

Obviously, just from like in the professional all the way down into grassroots-I was taught by a rugby player in PE and he kind of told us the unwritten rules one day one. That you do not act up at a referee you just accept his decision and walk away and continue to call him sir. And I've lived by that ever since. Yes, I've been frustrated at a referee, but I'll never question a referee's decision.

One's understanding of rugby culture created a standard of behavior that seemed to include how one should behave toward referees. So even when the coach or player believed a referee to be wrong or interpreted a situation differently from a referee, rugby culture held that the referee was still to be respected. The behavioral standards passed 
down through rugby culture influenced the way in which these 15 coaches perceived and engaged in referee abuse. KU recounted an example of rugby culture being upheld in an international test match:

I think there's a respect for each other, a respect for the referee. I don't know if you remember this or have ever seen this clip. There was a New Zealand vs. South Africa game and this was probably, if it wasn't in the 1990s it was in the very early 2000s. A drunken, 300-pound South African supporter thought it would be a good idea to get on the field and interact with the referee in a physical manner. And he was subsequently banned from all rugby games for life and you should have seen the state of him coming off the field, because both-it was at a scrum - both the New Zealand and South African pack sorted him out very, very quickly. So, you have two teams going at it as hard as they can, but once something interfered with what was culturally correct behavior, it was stopped immediately, right, because they respect each other, they respect the game, and they respect the referee. And with those things ... you can play really, really hard and you can still be respectful and still be sporting.

KU's example illustrates the ethos of rugby culture. All participants in the game are to be respected, and when that does not occur, it is the responsibility of others involved in the game to protect the sanctity of rugby culture. The situation KU described highlights the way coaches see rugby culture influencing perceptions about referee abuse and the likelihood to engage in abuse behaviors.

Of the 15 coaches, 13 also understood rugby culture by comparing it to the culture of other sports. According to NW:

I think for most Kiwis [New Zealanders] or Brits or South Africans we've very much been brought up with, [referee abuse is] unacceptable. It's like whatever the ref says goes. So, in Britain, we take pride and we separate ourselves from the soccer culture, the rugby culture is much, much different. You see soccer players harassing referees, hurrying referees on the field, you'd never see that out of a rugby player. You're brought up that way. You just don't answer the referee back. The only person that talks to the referee is the captain. So, that's when you grow up with it and I think if you grow up that way and then you become a coach then you start passing that same culture onto your players. 
The respect shown to all rugby participants is what coaches seem to believe differentiates rugby culture from the culture of other sports. To explain rugby culture, many coaches compared the sport of rugby to the sport of soccer. Coaches in this study made it explicitly clear that they did not want the rugby culture to become like the soccer culture. PZ explained:

You know you have to do a comparison any time you hear about referee abusing in rugby. You don't want to get like soccer. I think there's definitely been a swing towards it being more acceptable to open your mouth in some kind of way towards a referee. And I think that's, I mean, that's sort of a concern. I think it has definitely become more prevalent, in my opinion, on the pitch. I mean certainly if you go back-I could think about 10 maybe 15 years much more of it came from the sideline, so you might have coaches step over the line verbally more, or spectator step up over the line verbally. I think players are starting to come up a little bit in just the way they talk to the refs and I think the coaches are starting to get better in that perspective, but I think we just have to be very careful about it because traditionally we've been one of those sports where you know you have the 6'5" guy looking down at the ref saying: Yes, sir. Thank you, sir. Sorry, sir. And that's kind of what we don't want to get away from.

While rugby was understood to set a high standard in terms of respecting all aspects of the game, including the referee, soccer was seen to personify the expectation of negative and abusive behaviors against the referee. In this way, coaches felt that if a soccer culture were to be adopted, those embracing this culture would be more likely to accept referee abuse and more likely to engage in abuses themselves. The stark contrast between the two international sporting cultures underscores the influence of culture on a coach's perception of referee abuse. Beyond the comparison to soccer, many of the rugby coaches noted the differences between rugby culture, other American sports, and the American sporting culture. HS posited:

I think when you have people that are abusing referees you send the wrong message especially to young players that, again not trying to sound pompous here, but that we're like any other American sport where you know parents and players everybody yells at the referee and no matter what happens the referee is wrong. 
The differentiation between abuses against a referee seen in many American sports and the expected behaviors in rugby was a continuous topic of conversation. GR highlighted this notion:

I think with all the other sports it's part of the sporting make-up to aggressively go after the referee. I mean in baseball it's part of the culture that you have the coach standing there arguing with the home plate umpire kicking dirt on his shoe and getting thrown out of a game every now and then. In basketball it's actually a coaching tactic to take a technical foul at certain times to send a message to the referee. In football coaches are walking on the field, they're jawing at the sideline judge.

The juxtaposition between rugby culture and other American sporting cultures exemplified the way experiencing these described cultures may impact a coach's perception of referee abuse. In other sports, “They don't have the culture rugby brings, they have the culture of American sports where the man goes out and kicks dust on the plate in baseball the coaches can yell any profanity at the referee, basketball players and coaches yell at the referees in all of our major sports," said OY. The acceptance of referee abuse in American sport is something nearly every coach mentioned. In this way, rugby was perceived to be unique from the majority of other American sports. It was also noted by two coaches that some other sports, such as golf and sailing, also epitomize a culture of respect for the official in maintaining the etiquette standards of their sports. For the sport of rugby, the coaches noted that a key factor impacting their perception (and others' perceptions) of referee abuse was immersion in the rugby sporting culture. KU explained:

So, maybe coaches coming from other sports or having a short period of time in the sport of rugby, so maybe not being immersed in it their whole lives and being coached in a different way themselves where that behavior is almost allowed or even possibly the norm and then it gets passed on. Whereas I think in the better programs you see coaches that have been coaching and have been involved in the game to a higher level for longer periods of time. Even if they're just American referees they’ve been exposed to, sorry coaches, they've been exposed to 
themselves maybe playing abroad, so they're used to more of an international type culture.

The coaches highlighted how moving from other American sports to rugby may often require a cultural shift. It was noted that the uptake of the rugby culture would not happen instantly but rather the culture was something that needed to be taught to new participants. AL suggested:

It's got to be taught by those that have that already have that respect. I mean when you've got a new player coming in - and this is where we see it-you can't expect them to change overnight. They bring with them both the talent and the skills the love of sport that's coming with them from other sports but they're also bringing the baggage that goes along with it-demonizing opposition, abusing referees and those type things. So, whenever we get new people into the sport some of those are coming in, as you know, at 18, 19, 20, sometimes 23, or 24 years old. You know it's important for both the coaches and their peers, number one to set the standard - if you have somebody coming in and they're seeing the coach for berate players or berate a referee, guess what they're going to do. And emphasizing to them that this isn't any different than what I was doing in HS football, I guess I can still be doing that. So, the short answer is this is it rests on the shoulders of those that are already basically absorbed in the rugby culture, especially the coaches.

Most coaches felt that an exposure to rugby culture would ultimately serve as a deterrent to referee abuse. Those who were acquainted with rugby culture would perceive referee abuse to be wholly unacceptable in the sport. But as the sport continues to grow and new participants enter the game, the possibility of the degradation of rugby culture is an everpresent reality. NW explained:

So, obviously, quite a lot of coaches-most coaches-were playing here decades ago or when they had friends come over from England and introduce them to the sport or they traveled to England and saw it and started picking up on it, so that's when the sport sort of grew, so they started getting those values from Europe and sort of like Australia, New Zealand, where that sort of is the rugby community. Whereas if it's new coaches that are new to the sport-it could be a kid's dad or something like that - that hasn't grown up with rugby and is new to the rugby community he's not going to be aware of that environment where it is respectful and you're not going to shout abuse. So, he'll start doing bad examples himself when there's a game going on. He doesn't know that it's not right to be shouting 
at a referee in rugby. And obviously his players are going to see it and they're going to start following suit.

NW alluded to the fact that those who are new to the sport and have been immersed in the rugby culture may not understand that in rugby, unlike many other American sports, referee abuse is not to be accepted. So one's perception of referee abuse was significantly linked to the cultural understanding of the sport of rugby or the sporting cultures from which they have come. EP noted:

I think American culture is such that other sports have allowed and potentially condone these actions against officials and I think that has spilled over into the sport of rugby, which I don't think fits into the traditional American sport, right? It's not baseball, it's not football, hockey, or basketball, or soccer could be that fourth one, whatever. I think the want in our game to Americanize the game has allowed that to creep into rugby and I don't think it's a positive thing. I think there are lines that we need to draw as sports people in rugby and I think that falls on... I think we started to allow these things to happen and maybe our culture and society isn't as such that it's hard to look at another persona and say-that's unacceptable and we don't tolerate that here. And I think that it's at a point that we need to start doing that. I believe we should encourage and empower people to say that to one another, you know. And I think that will go a long way to curbing referee abuse, not letting it creep in any further than it has.

Within American sport, there tends to be a focus on winning, professionalization, and an underlying belief that the referee is a barrier to be overcome. HS explained, "We want to grow the sport, we want to grow the sport, we want to grow the sport, but what we kind of realize is that when we grow the sport and we take out athletes from other sports is we adopt those customs." OY mirrored HS's belief: "The win and the loss wasn't always the barometer or the measuring stick. Sometimes it's how the games is played and respected and sometimes that goes out the window when we Americanize it." New participants facilitate the Americanization of rugby, ultimately influencing rugby culture. As such, coaches have highlighted the importance of being cognizant of the behaviors brought into rugby from other American sports. All 15 coaches expressed culture-inclusive of rugby 
culture, American sporting culture, and soccer culture — as a reoccurring theme and believed that culture was incredibly influential on referee abuse behaviors and perceptions. JA's statement summarized the participants' perspective well: "So, everyone's got their own cultural variances, we don't need to be anybody else's culture, we need to be ours. We need to show in a growing and emerging sport that we can just be respectful."

In answering the second research question-What factors influence the perception of referee abuse?-five themes emerged. The Division I-A rugby coaches perceived referee abuse to be influenced by the following factors: (1) the personal characteristics/philosophy of the coach, (2) the coach's relationship with referees, (3) social influencers, (4) organizational role expectations, and (5) culture. These factors helped to explain how coaches perceive referee abuse in the rugby sporting context and were also closely related to the solutions that coaches offered in attempting to curb referee abuse.

\section{Solutions}

In addition to exploring collegiate rugby coaches' perceptions of referee abuse and the factors that contribute to these perceptions, the third research question sought to inquire about the actions that may alleviate referee abuse. When suggesting solutions that may help reduce referee abuse in rugby, many of the coaches referred to the factors that influenced their own abuse perceptions and behaviors. Referee abuse was generally believed to be a topic that had not been appropriately addressed within Division I-A rugby or within the larger USA Rugby organization. Despite this, all 15 coaches thought that referee abuse could be fought on various fronts and addressed in numerous ways. To 
address referee abuse, the coaches interviewed suggested that all rugby stakeholders must enhance standards, focus on education, and work toward collaboration.

\section{Enhanced Organizational Regulations and Protocols}

When considering how to deter referee abuse, 12 of the coaches proposed enhanced organizational standards. Such standards might include increased awareness and follow-through with referee abuse sanctions and technical zone protocols, along with the provision of adequate referee coverage for matches, ensuring a team of three for each event.

The coaches noted that while consequences for referee abuse existed, few had ever seen these consequences enforced. The leniency around referee abuse standards was cause for concern for many of the coaches. NW explained:

I don't see many examples, at least publicly, of punishments being handed down, of coaches being held accountable. I mean the same coach that I mentioned earlier and the foul language on the sideline that always seemed to be that way. Always seem to be coaching year after year after year after year. And that's a problem with the coaching fraternity, there was a bit of an acceptance of it. And, you know I think that's-I'm not saying get him out of the game-I'm just saying when we step over the line as coaches, I think we need to be held accountable the same way a player was held accountable if they're sent off. There should be some sort of ban. It's been a long time since I can recall any of that sort of stuff happening. You know us coaches, step over the line. So, whether we mean it or not there should be some sort of accountability at least a scale of how bad was this? What did the ref say and was their opinion on the matter? And is there any sort of consequence for the coach, because I think that's really the message you said which is-we know you can make mistakes but there is still a consequence for a mistake for a coach too. Sort of make the referees feel that they've got the backing of coaching community as well as sort of the admin community.

Without any fear of reprisal, referee abuse behaviors seemed to be overlooked by both the coaching and administrative communities. In addition to coaches rarely seeing consequences for abuse behaviors, few were aware what those consequences might be. 
DO posited, "I don't know exactly what the punishments are. I know that some do existI would assume a suspension of some kind or some kind of fine, but I don't know off the top of my head." Because the coaches were unaware of the consequences that may result from referee abuse and did not see consequences being enacted for abuses within USA Rugby, they believed that abuse behaviors continued to be unfettered. There was one coach who expressed dissent from the majority. This coach was knowledgeable of consequences for referee abuse and deemed them to be extremely stringent. OY noted, "It's now if you look at a ref wrong it's like a seven-month ban and you lose two children." The hyperbolic nature of this commentary suggested that while OY was aware of the consequences of referee abuse, OY did not find them particularly fitting or beneficial to the game. OY went on to explain that, despite the existence of consequences, referee abuse continues to be widespread. At the time of the study, six of the 15 coaches suggested that appropriately structured and enforced consequences would decrease one's proclivity to engage in abuse behaviors. NW explained:

USAR will have to start implementing policies in place so if a player is abusing the referee there are sanctions and the referee can then record it. And if they start to see players being banned for a match or two players are going to start thinking twice before going ahead and abusing him. Because for most college teams there aren't a lot of games in the year, you're playing maybe 10-12 games in a season, so if you're getting a 2-3 game ban that's quite a lot of your season out just because you lost your frustration.

NW continued:

So, if we start setting that standard where we are seeing these people getting reprimanded for referee abuse it is going to stop. I know in all grassroots rugby in England, and even grassroots sports in general, when you go on the rugby field or soccer fields and stuff like that there's usually a sign for parents as they walk in saying referee abuse is not tolerated that if you do it you'll be kicked off the park. So, I think having that sort of standard there, that visible warning for what you do-there is a consequence for it. 
NW suggested that visual reminders be utilized on the field to set the standard and ensure that the behavioral expectations for the competition were clear. If coaches, players, and spectators were made aware of the standard and a reminder was provided at every pitch, the expectation would become that referee abuse is not tolerated. EP recounted a story in which a coach was removed from the playing enclosure by the referee, having been shown a red card for referee abuse. As it turns out, the coach had received a total of four red cards, having previously been ejected from four games. Despite that, the particular competition had no prescribed recourse for the coach's referee abuse behaviors. The coach could have been removed from all of the games and would still have been able to continue coaching. Instances like that highlighted the importance of creating organizational consequences and ensuring that all parties are aware of these policies. Many of the coaches believed that setting a clear behavioral standard regarding referee abuse may serve as a valuable means to dissuade abuse behaviors.

In addition to improving the awareness of behavioral standards and sanctions for referee abuse, numerous coaches also suggested that the adherence to current regulations within the organization be paramount. At the time of the study, there were Division I-A requirements regarding the use of a technical zone. The technical zone is a space that separates the two teams from one another and provides boundaries for the sporting participants. Despite the best intentions of the governing body, coaches reported that technical zones were not utilized regularly within the Division I-A competition. EP explained:

Well, first and foremost there's no adherence to any sort of regulations. There's technical zones from USA Rugby, our national governing body, that have been out since 2014, but I'm still not seeing that in certain areas, specifically in DI-A. We're playing a team in the [location] championships and our coaching staff is 
standing where our technical zone would be, our players are in the bench area where that would be and the opposing team's coach is just walking in front, standing next to us, screaming things at his players, screaming things at his referee, and I'm just standing there like I have a water bottle and I just want to squirt you so that you go back to your area. You're "harshing my mellow," I'm trying to coach, I'm trying to focus on my players. Not the referee. Not you. Not your inappropriate behavior.

The lack of adherence to the requirement for technical zones created a circumstance on the sidelines where players and coaches of opposing teams were wandering into one another's spaces. EP continued:

I think the technical zones would benefit us, because I've got the opposing team's coach standing next to me, in what would be my technical zone, screaming things at the referee and how does the referee know that it's not a [my team] coach? You know? It's sort of like, not my monkeys, not my circus, get over in your box. And that's what they're there for, right. They're there so players can play, coaches can coach, medical staff can do their job and they know where, if something-if there is abuse or there is verbal engagement - they know where it's coming from. If you don't have that there are no rules, it's just chaos.

Additionally, the coaches' and players' proximity to the field when technical zones are not enforced became a cause for concern. PZ noted, "There's a closeness of the sideline to the field which allows people to get more caught up in it, which leads them to do or say things that they might regret afterwards." The coaches believe that this proximity, if uncontrolled or unregulated, could impact perceptions of and, ultimately, participation in referee abuse. Thus, to curb abuse behaviors, the use of a technical zone should be enforced.

Beyond enhancing behavioral standards and requiring the use of a technical zone, coaches also suggested that assistant referees should be assigned for all competitions at the Division I-A level. Of the coaches, $40 \%$ reported that in most cases, they were not assigned assistant referees. That means that the center referee was responsible for controlling the behavior of 30 players without any assistance. The coaches noted that the 
referee has no support and thus was less likely to catch behaviors that coaches may deem

to be unfair or unsafe. LV explained:

If you have two touch judges that are actual referees and a referee that helps catch a lot more things. But, that's the thing, I think I brought it up on our last call-I watched from where I was my flaker get punched in the face and you've got a touch judge starring in that direction, but if your touch judges don't have the confidence to make a decision or point something out. And those are the type of things where my blood gets boiling, and whatever your definition of referee abuse is I might yell some things towards the referee that aren't very nice because my kid's getting punched in the face and nothing is happening.

Ensuring that each Division I-A match was assigned a team of three officials provides additional sets of eyes, which could help to identify referee errors and ensure consistency in calls. MX noted:

The hardest thing about refereeing that I find is there's only one ref. I think that if we would use touch judge more effectively in the USA, it's more important for us because our referees aren't that good. The hardest thing for the ref is he's out there trying to watch that ball and the breakdown area and then he's got to look, he's got to take a step back and turn his head back totally the other direction, after he's figured out that breakdown, now he's got to turn his head and look totally across the field and make sure everybody is onside. And I think that if we would use our touch judges more effectively, we could have a lower skilled referee do a better job of reffing the breakdown and let the touch judge take care of the offsides. Now, again we already have a shortage, so my solution, I think I've told you the biggest thing they've got to do is get the players involved and start giving free classes and make every player in the USA involved a referee. But, they gotta make that free. You can't be trying to turn it into a money maker.

Providing a team of three could help reduce concerns over incorrect calls and safety issues in addition to providing the referee with moral support. Concerning enhanced regulations or protocols, $80 \%$ of the coaches believed they could help reduce referee abuse in collegiate rugby.

\section{Education and Training}

Education and training were the most prominently occurring solutions put forth by the coaches in this study. All 15 coaches suggested that education and training must 
occur for all stakeholders in the game, including referees, coaches, athletes, and

spectators. They also highlighted the importance of collaborating with other stakeholders

throughout the educational process. For the coaches, it was about education for everyone.

Educate yourself, educate your athletes, educate your supporters. I just feel like the more people know the less bad they do. For me I try to make sure that my athletes are educated, but like we were talking about earlier, it's important for me to walk the walk as much as I talk the talk. So, I like to be a good example. Calm down. Get educated. Be a good example. (DO)

The consensus was that all rugby stakeholders could use additional education to be more proficient in their roles and to help directly impact their perceptions and behaviors related to referee abuse. Many coaches suggested that education of fans and spectators was incredibly important in helping to deter referee abuse. In America, the coaches noted that most of the fans lacked an understanding of both the rules and culture of the game.

I think sometimes we've really got to educate folks on the culture of how we've always handled our referees and how we've always had a relationship with them. In general, we will always call the ref sir/ma'am or whatever they've asked us to call them beforehand, and I think that's always been sort of part of how officials have been respected in the game. ... And it's really tough because you get these parents who come to two games a season and it's really tough to tell them okay, we get that you're invested in the performance of your kid, but it's tough to tell them that's not how we do it because how do you build a relationship with these parents? Now, the parents that we see every week who come on the road with us - they're part of the culture. They're part of educating the other parents as well, and that's really your sweet spot. (PZ)

Here, $\mathrm{PZ}$ expressed the importance of educating parents on the rugby culture; in this way, parents and spectators become familiar with the traditions and practices of rugby, inclusive of respecting the referee. Coaches also suggested codes of conducts for spectators that are posted at the field or hosting a pre-season meeting for parents and spectators to introduce them to the rules of rugby and the expectations of the rugby culture. 
HS mused:

What I'd really love to see in a dream scenario is if we could have actually someone in the stands, maybe with an accent so that people would listen to them, saying calm down folks that's not a knock on or that's not a high tackle, he made the right call. Something along those lines, because sometimes it's kind of the crowd that builds it up. So you can educate everybody so that people aren't so reactionary and ignorant, I guess. And then just getting experience with rugby as a sport that's been around so that people know what to expect and know what the standards are internationally.

In addition to educating parents and spectators, coaches noted that it should be a priority to enhance coach education. At the time of the study, many of the coaches found USA Rugby's coach education process to be of little value. Additionally, the trainings failed to focus on referee abuse. The coaches believed that USA Rugby coach education should set the standard regarding behavioral expectations toward referees. It would be at this time that the definition of referee abuse, including examples, would be shared. Additionally, consequences for referee abuse could be outlined. Beyond a mere recitation of expectations and consequences, OY suggested that it would be important to explain why. Why should coaches care about referee abuse? How is this issue impacting the game and impacting the coach?

I feel like they were checking a box. Hey, don't abuse refs. When you tell your kids something you tell them why-you explain it. So, you have to tell them why-well, people aren't going to want to ref. (OY)

Explaining the rationale for various regulations and standards was important to the coaches in order to enhance the value of referee abuse training included in coach education. HS explained:

I think it's education for the coach, the coach themselves. I'm trying to think of a clever way to approach this. It's almost like with sexual harassment where a lot of people don't understand that what they're doing is abuse. And need to be educated on that. And then once you get enlightened yourself you can pass that on to other people around you. 
In this way, coaches could be trained on both the definition and the impacts of referee abuse. The coaches' understanding of referee abuse could then be passed down to their players. As KU explained:

Because if we're getting into our kids and our coaches that are coaching our 5year-olds and our 6-year-olds and our U12s that early that this is how you behave and this is how you treat a referee and these are the standards that are set, by the time you come through we should have a solid platform in place. Whereas if we've come through a youth program where we've been refereed poorly, we've talked to referees in a manner that's not acceptable, then that becomes the norm.

As coaches are continually exposed to and reminded of USA Rugby's referee abuse standards, the behavioral expectations regarding referee abuse may become more positively normalized.

Beyond the inclusion of referee abuse as a topic in general coach education within the sport, 12 of coaches suggested that coaches should be required to become certified referees as part of their educational journey. The requirement for referee certification takes the awareness of referee abuse one step further. In this case, coaches would be asked to walk in referees' shoes and experience what they have experienced. CN suggested:

I think for coaches it would be awesome if it incorporated or some of their certification was to actually take a referee course of some description, whether it be a level 1 course or whatever. Have some element of - go and referee the game then and see how you do it sort of thing - as part of their certification process. Or as part of their continuing education process, part of that. I think that would be really valuable, it would also help their programs because they could actually help their players, instead of teaching them to do the wrong thing they could teach them to do the right thing. I think that would be a really valuable thing.

The benefits of requiring coaches to participate in an officiating certification course are multi-faceted; not only could the coaches have an understanding of the laws and how they may be called to educate their players, but this understanding may also translate to 
empathy for the referee's decisions. In addition, putting oneself in the referee's shoes may help to illuminate the difficulties of managing 30 players as a single individual. DO completed a referee course and found it to be incredibly valuable. "For me I spent a lot of my time as a young coach doing a lot of judging without actually being a certified referee. So honestly since I've taken the course, I do a lot less of that." DO continued on to explain:

It's really easy for me to be like "oh I don't like that or that call is wrong," having some context around it and proper training I was able to judge myself was that call right or wrong according to the law book? Yes or no. If it wasn't, ok. Well, what were the factors that might be influencing their call - is the game really fast paced? Are they, do they not have a lot of experience? Were they just not in the right position? It's just easier for me to think a little more critically about what they might be going through per say.

The ability to understand what the referee is experiencing may help to reduce frustrations felt by the coach during a match. GR has found that being a certified official does, indeed, impact GR's approach to referee abuse. "I have some empathy for what they do, and I can approach them in a way that I think they appreciate less combative and more hey I just have a question as to how to advise my team to ensure that we're not on the wrong side of your whistle." GR's officiating experiences have allowed GR to approach a referee in a new way. Instead of seeing the referee as an obstacle that must be overcome, the coaches would be more able to empathize with the difficulty of the position and thus their perceptions of abuse would change accordingly. DB put it very frankly: "Try getting abused." DB felt that if coaches are asked to become certified referees and referee games, they will experience abuse.

The coaches believed that the same benefits from their certification as officials could be replicated for players. Many coaches promoted officiating to their players and 
helped provide funding for them to become certified referees. Further, Division I-A rugby

has also enacted a new officiating certification requirement for each of their programs, as

GR explained:

We have an initiative with DI-A that every team is required to get five students certified as referees. We've had some schools like Santa Clara got their entire freshman class, 25 people, certified. Their goal now is that all their freshman will get certified, so in 4 years' time they'll have pretty close to $100 \%$ of their players certified as referees. We've got 138 so far this year in DI-A that have been certified as refs. We have an aggressive goal of 350 , but we know that even if we fall short we know that $100+, 200$ referees is a good accomplishment for a first go at it.

The Division I-A competition is already beginning to enact some of the solutions that the coaches felt would be most valuable in curbing referee abuse. There seemed to be remarkable support for such an initiative. FQ explained:

I think college coaches should be encouraging their players to get involved in refereeing. Because guess what? Not every college player is going to be going off and playing the MLR, the USA Eagles, the Collegiate All-Americans. But, you know what those athletes can go and make an immediate impact on refereeing and they're far more likely to get to a higher-level refereeing than they are as a player. So, I think encouraging that, but stressing it to them that hey you guys aren't the problem here, you can be a part of the solution. Like us in that leadership role to do that and to send that message would be huge.

While coaches felt that referee certification was beneficial and many programs have already begun to support the certification of their players, putting those skills to use was also valuable. FQ explained:

We try to get five kids inside of our team certified as referees every year and then like we make them actively referee. We make them actively referee our in-game scrimmages and practice scrimmages, and we make sure that we get them actual games to referee. And we come at it from the angles like, "Hey man like this is a dude that you share a house with or you hang out with this is a friend or he's inside your unity group so why are you treating him like that? You know like that you shouldn't be talking to him to begin with." 
NW also felt that challenging players to officiate can be beneficial in deterring referee abuse.

I think what you could do is then just give them more experiences of refereeing games, so possibly if you're a program that hosts like HS camps and MS camps, you can get those players to referee touch games or whatever you're doing and just start getting them more exposure to what it's really like. And hopefully they'll realize, actually, this isn't fun being shouted abuse at so maybe I'll change my ways.

The combination of education and truly walking in referees' shoes seemed to be the solution that coaches believe would be beneficial in reducing referee abuse.

In addition to the education of parents, coaches, and players, the interviewees also suggested that referee education and training must be improved. Referee education and training provide the foundation for the quality of the referee at various USA Rugby competitions. At the time of this study, 10 of the coaches found the education and training of referees to be wholly inadequate, which led to perceptions of bias, safety issues, frustration, and, subsequently, referee abuse. FQ noted a perception of the current referee education structure:

Where like you know the referee side of things. I don't think that there is anything out there and honestly don't think there is anything out there besides a bullet pointed e-mail that's sent out weekly about expectations around calls where that's not cutting it. It was like trying to manage referees through essentially a spam email where that's just unprofessional and unhelpful to the game. I use an example with [my elite rugby academy] all the time. It's a triangle - at the top of that triangle are the players. The two base points are the referees and the coaches. And if one of those base points fails you know everybody else fails. Refereeing fails, players fail, coaches fail. But most importantly it affects the coach. And right now, you're seeing a lot of money being pumped into player coach development and time. There's not any being put into the other side the other side of that triangle-referees. So, I think we as a rugby community are kind of letting ourselves down by not investing in it, but the people who should be investing in it aren't doing a good job. And I think a good model people should be following is what USA Football does with their referee education which like you know clips or articles there's great examples of it that are almost reviewed and updated on a weekly to monthly basis. 
The coaches understood there to be little continuing education for referees beyond their certification courses. To actively participate as a rugby referee, one must pass an introductory officiating certification course, pass regular background checks, and remain registered as a referee with the national governing body. There are no ancillary requirements for referee continuing education, nor are there many opportunities for additional education should the referee want to pursue them. JA was astonished to learn about the lack of systemization in continuing education for referees. They were running a scrum clinic with both referees and coaches, "and we found out that there was no model, no model in place. I mean are you aware of that? How do we teach the front row? How do we teach engagement? There was zero!" The lack of a set standard for educating referees creates the potential for coaches to see different officiating standards every match.

Beyond the requirement of a level one certification course, referee continuing education is undertaken at the discretion of the individual. GR suggested:

I think we have to find a way that referees are getting some type of training and feedback throughout the week, that they're not just walking up on a Saturday, you know because that's their performance, as well. A combination of fitness, video analysis, I think that for referees attending training should just be a normal part of their season as it is for coaches and players. Whether it's one training session a week, one fitness session-I don't know what the right number is, but . . . and they have to watch video. They have to find some way, because video doesn't lie. That's what we find as coaches. I could tell an athlete until I'm blue in the face that they're going in too high, and they think differently because their mind is telling them I'm getting low, I'm trying to get low, but as soon as they see the video, they say oh wow, I'm coming in too high. And I think the same thing can exist for referees-you can tell them one thing but as soon as they see the videoit doesn't lie. I think that's the time people really own it and acknowledge what they're doing. 
Many of the coaches agreed with GR's perspective that referees often do not engage in education or training outside of their match day requirements. Coaches did acknowledge that officiating was a nominally paid position and the majority of officials do maintain other employment. Despite these setbacks, coaches implored referees to invest in their own education and training. NW explained:

So, I mean that comes down to how much training are they getting, how much experience are they getting, how much evaluating are they getting off their skills? If they're simply just turning up on Saturday refereeing a game and then leaving, if they're not getting access to the game footage to view themselves ... I mean I've never had a referee say can I get the game footage, so I can evaluate myself, so I know that's not necessarily happening.

The coaches interviewed suggested that referees must hold themselves accountable for their own development.

To deter referee abuse, all 15 coaches suggested the improved education of various rugby stakeholder groups. In addition, they suggested that many of these educational endeavors should be collaborative. For coaches, creating consistency between coach and referee perceptions began with a shared education. JA explained:

How much resources do we have out there for referees that's free? How much of it is pushed to them or do they have to go and look for it? How much of it is sent to them? How much referee resources when it's sent to referees is being sent to the coaches? So, let's say for example, let's just take it out of my region to the East Coast, we're going to send out a bulletin of information to all under-12 coaches on the East Coast, so this information is also going to be sent to the referees and this information is on how you're going to referee the game. Rather than it just being sent to the referee is it being sent to the coaches? So now everybody's on the same page. These are the behavioral guidelines that the referees are setting out. Here's what you need to expect at games. We want the fields to be fenced, we want technical zones, we want this, we want that, we want this. Is that being sent to the clubs, so is everyone on the same page with the same information, consistently, across the country? 
GR mirrored the need for consistency and collaboration in educational resources and programming:

I think that part of referee ongoing training and development should be joint with coaches. The continuing education should be part of it should be working with your coaching peers who are also giving you feedback on your game or hey this is what we're doing at the breakdown, and this is why, this is how we see it. So, the referee can then say I never thought about us our way or no, this is why that's absolutely wrong. Here's how it violates the law. You know there is some interaction and back and forth and a little bit better understanding. But, I think educating referees separately and educating coaches separately just continues to build that gulf and that distance between the two.

Training and education were seen as opportunities to foster collaboration among rugby constituencies. Holistically, the coaches held that education and training were key components in the reduction of referee abuse.

\section{Collaboration}

As previously addressed, the coaches believed that enhanced policies/procedures and improved training and education programs could help to deter referee abuse. Additionally, all 15 coaches suggested that referee abuse may be deterred through enhanced collaboration between the two groups. FQ noted:

I think it's a big problem. And I think that's only going to get worse if we don't put some policies and protocols, but I think we need to actively encourage more interaction between referees and coaches. We've got to break down those barriers.

Like FQ, the other coaches also prioritized opportunities in which coaches and referees could come together to work on various aspects of the game. GR contended:

I have not been to enough opportunities where coaches are involved in the referee training process or engaged with referees or speaking to referees. Typically, coaches do training on their own. Referees do training on their own. The two groups rarely mix, so I think that there's a lot of misunderstandings and misconceptions about what's happening in both camps. I get to speak to a lot of referees individually as a coach and as an administrator. So, I think I'm kind of lucky in that regard, so one on one I get to talk to referees, but in a group setting it's something that I would love to do more of. 
Joint training was one of the primary ways identified as a means to break down the barriers between referees and coaches. The coaches suggested that this may include certification courses, annual general meetings, forums, or even having the referees join the coaches at practices as part of their own training. A great example of how coaches and referees could work together to facilitate a better working environment for referees came from KU:

So, I had a referee come in and do a training session and then they had a referee do another training session on scrummaging up at [a University], like a week ago with DI-A referees. So, the coaches are working alongside referees now to try and raise standards collaboratively and I think that can help referee abuse-building relationships can stop referee abuse. It can be a helping thing massively because you start to get mutual respect for both parties.

In this instance, some of the Division I-A coaches were not content with the way the scrum had been officiated in their recent matches. Instead of complaining about the quality of officials, these coaches took it upon themselves to make a change. They were able to develop multiple opportunities where referees and coaches could come together in order to discuss relevant issues in today's game of rugby. For the coaches, changes in perceptions come from the sharing of ideas. KU questioned:

What are you going to gain by threatening or abusing a referee? If you want to change that person's opinion in the future are you going to do it by having a respectful conversation, looking at film together, discussing the laws together, discussing the interpretations, over a beer or whatever it is.

Opening the lines of communication between referees and coaches allowed for collaboration and the building of relationships. NW, like other coaches, suggested collaboration in training but also felt that the opportunity to be social with refs would truly facilitate relationship development. NW explained: 
So, I think if you can have referees come into your training environment and assist you and answer players' questions that's going to be a huge thing for everybody. And then also being social after games. Inviting the referees, if you do have food post game, inviting the referees to stay around and have some food with the team and answer any questions about the game or what their views on stuff are. More interaction so they're not just this person that stands in the middle of the field, they're actually a human person and you get to know them personally.

Six coaches also highlighted the value of interaction between the coaches and referees at a post-match function. The post-match function in rugby was viewed as an opportunity for all rugby stakeholders to come together to celebrate their love for the sport. It is at this time that team affiliations or rugby roles are pushed aside, and everyone shares a meal together as a family. Coaches in Division I-A rugby find the continuation of this tradition to be key in developing relationships among all parties involved in the game. According to AL:

[The post-match function] was where you retained that respect for the other team because you could see guys that you played against 40 years ago and they come up because remember that we actually can't remember who won the game right. You remember the time after the game where you spent a couple two or three hours with them afterwards over burgers or beer or Cokes or whatever we you drink. And I think that culture basically promoted the idea of the respect the referee that it he was just another member on the pitch that was helping the game move forward.

Creating a personal relationship with referees where they were understood as a valuable part of the game was seen as key in reducing abuse behaviors. Though the coaches offered ways to facilitate the development of relationships with referees, the relational outcome was always more important than the way in which the outcome was achieved. Collaboration between the two parties and ultimately developed relationships were believed to be a viable solution to reduce referee abuse. 


\section{Barriers to Enacting Solutions}

Throughout the course of this study, the coaches proposed numerous solutions that would help curtail referee abuse, focusing on the importance of education, collaboration, and the development of practical policies and standards. Despite their enthusiasm for providing strategies to reduce referee abuse, the coaches noted that enacting those solutions required the navigation of numerous obstacles. One of the primary barriers noted by 11 coaches was a lack of financial resources. The lack of resources was believed to inhibit USA Rugby from creating additional educational resources for both referees and coaches. Further, the coaches expressed that there were few full-time paid employees within the Division I-A rugby sporting structure. As such, the incentive to solicit additional work and advent new programs was limited. KU noted:

We've got massive financial problems, we've also got a massive geographic problem. Our country is huge, so how are we going to get our resources to small areas where we've got a handful of small pocket of referees that aren't able to get training. So, they now use the funding and say to those guys, okay, we can't get a trainer to you, but we'll subsidize flights for you to come to NY or Boston or California. So, it comes down to money, right. We've got to have resources, got to have money.

Without money, many individuals would be unable to access the educational opportunities that the coaches proposed. KU also highlighted the difficulty of geographic proximity within the United States. The United States is a large country, and rugby is geographically spread across the entire country. The sheer distance between many programs and their counterparts creates isolation. It is difficult to build relationships as the coaches have suggested if traveling to a team's practice would require a 100-mile drive. It is challenging to host joint educational opportunities when doing so may require multi-hour flights. 
In addition to concerns regarding money and geographic distance, the coaches noted that rugby personnel also tended to be an obstacle. Seven of the coaches suggested that there was poor leadership within the USA Rugby organization. As such, the national governing body may not prioritize the reduction of referee abuse. The lack of leadership was also believed to be compounded by numerous political issues plaguing the national governing body and its subsidiaries. The coaches thought that many of the individuals in power would be reticent to enact change simply because change may result in relinquishing some of their authority. EP noted:

I think refereeing in the society model is the last wild west frontier of American rugby. I think ego plays into a lot of what we do on the coaching level, the administrator level, and it definitely plays into the refereeing.

$\mathrm{CN}$ mirrored these sentiments:

The game has changed a lot now that there's a professional game it kind of feels like everybody's out there to see how much of the pie they can get, instead of how much of the pie they can give. And there's a lot of egos out there who would like to think they know all or want to tell everybody else how to do it-and it's like get over yourself.

In addition to the potential for political issues fueled by an individual's ego, the coaches also noted that another personnel issue was that of the volunteer nature of rugby. Within the sport of rugby, specifically at the Division I-A level, many of the administrators and coaches are volunteers. OY said, "There's probably not more than $20 \%$ that get paid. I would say that $20 \%$ would probably be high. I know that we don't have a paid position in our conference. We have like a nine-team conference.” As such, many of the coaches and administrators hold full-time jobs external to coaching rugby. The time dedicated to the sport of rugby already detracts from the time that they are able to spend with their families and friends or pursuing hobbies. Thus, the voluntary nature of Division I-A 
rugby limits the time and energy that coaches have for implementing the many strategies that they have suggested. LV noted:

Probably the fact that the majority of all coaches and referees are all volunteers. And they're already giving up tons of their time away from family and jobs and all that so it's tough to force anyone to do anything. It would all be at will, so I think that's tough.

The volunteer base that oversees Division I-A rugby is inhibited by a lack of time to enact many of the policies that they have suggested.

Despite the constraints of money, geographic spread, politics, and volunteerism, the coaches remain optimistic that with commitment from the coaching, administrative, and officiating communities, they can advent change regarding referee abuse. They were confident that their peers would support additional education requirements. They believed that their conferences and leagues would be willing to create vision statements and policies regarding referee abuse. In addition, they were confident that with proactivity from the coaching community, they could facilitate the development of relationships and collaboration opportunities between referees and coaches. The next step was simply to take a step.

\section{Summary of Findings}

The 12 emergent themes presented in the finding sections of this paper express the multi-faceted understandings of referee abuse presented by 15 Division I-A rugby coaches. The findings were presented using verbatim quotes from the participants in order to allow the reader to become immersed in the coaches' understandings of referee abuse. For the purposes of this chapter, the aforementioned 12 themes were organized to answer each research question posed: 
Q1 How do men's Division I-A collegiate rugby coaches perceive referee abuse within the U.S. rugby sporting structure?

Q2 What factors contribute to Division I-A collegiate rugby coaches' perceptions of referee abuse?

Q3 From the perspective of a men's collegiate rugby coach, what actions may alleviate the problem of referee abuse in the college arena?

In answering the first research question, the findings demonstrate that the coaches perceived referee abuse to be difficult to define, frequently occurring, and responsible for numerous detrimental outcomes. These perceptions were founded upon their own lived experiences and organized through the continual visiting and revisiting of the data. To explore the second research question, the aforementioned perceptions of abuse were found to be impacted by a multitude of factors, including personal characteristics, relationships with referees, social influences, organizational expectations of the referee, and culture. A coach's understanding of referee abuse may be influenced by one or more of those factors at various times, and it is important to note that one's perception of abuse is malleable. In answering the third research question, the coaches believed that there were several solutions that could help to deter referee abuse in the sport of rugby. These solutions included the enhancement of regulations and protocols, increased education and training, and the opportunity for improved collaboration between coaches and referees. When speaking about solutions to referee abuse, the coaches were mindful of a number of barriers that inhibited the implementation of the aforementioned solutions, yet they remained hopeful that proactive rugby stakeholders could overcome all odds. 


\section{CHAPTER V}

\section{DISCUSSION}

Utilizing a phenomenological approach, this study employed semi-structured interviews with 30 Division I-A coaches to describe the lived experience of referee abuse within the rugby sporting structure. The purpose of the study was to explore (a) Division I-A rugby coaches' perceptions of referee abuse, (b) the factors that contribute to these perceptions of referee abuse, and (c) solutions that may help mitigate referee abuse behaviors. Prior research on referee abuse had primarily explored the perspective of the referee. This study expanded upon the existing literature by providing coaches' perspectives, identifying factors that may influence referee abuse and actionable solutions that may help deter referee abuse behaviors. Throughout this chapter, the findings are related to relevant literature and the theoretical model that framed this study. Following the theoretical interpretations, practical implications are presented. The chapter also includes a discussion of the study's limitations and the researcher's recommendations for future research.

\section{Review of the Research Problem}

Thus far, referee abuse has primarily been considered from the perspective of the referee, so this study sought to extend the literature considering referee abuse through the lens of Division I Collegiate rugby coaches. Referee abuse is a pervasive problem within 
all sport settings (e.g., Dell et al., 2016; Rayner et al., 2016; Walters et al., 2016), resulting in numerous challenges for sporting organizations (e.g., Dell et al., 2016; Ridinger, 2015). Not only does referee abuse impact the individual, it also adds to the existing concern of a referee shortage (e.g., Pappas, 2016; Sabaini, 2001). In sporting leagues across the United States, including the sport of rugby, administrators are faced with a lack of referees (e.g., Cuskelly et al., 2004; Pappas, 2016), and some researchers have suggested that this referee shortage may be attributed to referee abuse or related factors (e.g., Anshel \& Weinberg, 1995; Dell et al., 2016; Rainey, 1995; Warner et al., 2013). As previously noted, referee abuse had primarily been explored from the perspective of the referee. While the perspective of the referee is valuable, the researcher contended that the perspectives of additional sporting stakeholders must also be considered. As such, the study explored coaches' perspectives of referee abuse in rugby in order to enhance the holistic understanding of the issue of referee abuse. The factors that influence a coach's perceptions and potential solutions for deterring referee abuse were explored.

\section{Perceptions of Referee Abuse}

The coaches interviewed perceived referee abuse to be difficult to define, frequently occurring, and related to numerous negative outcomes. The perceptions of the coaches were reflective of their own experiences and thus expressed their own understandings of referee abuse. Despite the personal nature of each coach's understanding, when considered in aggregate, the emergent themes can be viewed through the lens of existing literature. 
Among the coaches, there was consensus that referee abuse should never be warranted within the sport of rugby. Despite this firmly held belief, differentiating between abuse behaviors and non-abuse behaviors appeared to be difficult. The coaches were clearly able to identify physical abuse behaviors but struggled to determine which verbal condonations of the referee would be categorized as abuse. The difficulty of defining abuse has been noted in other contexts, including emotional abuse of children (e.g., Glaser, 2002; O’Hagan, 1995), sexual abuse of children (Haugaard, 2000), and family violence (Malley-Morrison \& Hines, 2004). Haugaard (2000) noted that in the case of child sexual abuse, the term has never been "unequivocally defined" (p. 1036). Without a broadly accepted definition, research and advocacy efforts have been hindered (Haugaard, 2000). The same problem existed for the coaches in this study as they attempted to verbalize their perceptions of referee abuse. While many were able to identify and define physical abuse, conceptualizing verbal abuse became a point of consternation. Many were initially able to present their perceived definitions of verbal referee abuse but during the interviews wavered about instances and behaviors that would be included or excluded. Because the coaches did not have knowledge of a universal definition of abuse provided by USA Rugby, they were left to conceptualize referee abuse based upon their own working perceptions.

Physical referee abuse, as explained by the coaches, was seen to encompass any negative physical contact with a referee. The coaches' perceptions aligned with the International Olympic Committee's (IOC's) recently published consensus statement about harassment and abuse in sport for athletes (Mountjoy et al., 2016). The IOC defined physical abuse as "non-accidental trauma or physical injury caused by punching, 
beating, kicking, biting, burning or otherwise harming an athlete" (Mountjoy et al., 2016). In this definition, it is important to note that only harassment and abuses related to athletes were discussed. Abuses related to other sporting stakeholders, including referees, were lacking. Despite overlooking other sporting stakeholders, the IOC's definition of physical abuse aligns well with the definition outlined by the rugby coaches in this study. Because physical abuse is commonly understood to require physical contact, defining the concept within the context of rugby referee abuse was easier as well. Unlike physical referee abuse, conceptualizing other types of abuses, including verbal abuse, posed more of a challenge.

In many instances, non-physical interactions against a referee have not been labeled as abuse (Brackenridge et al., 2005), which has created a standard that increased the difficulty in defining verbal abuse behaviors. According to the National Association of Sport Officials, only 21 U.S. states have legislation in place that specifically designates attacks on sports officials as an offense (NASO, 2018). Yet even these legal definitions vary from state to state. Nineteen of the aforementioned states utilize legislation designating referee assault as a criminal charge, while two designate referee assault as a civil action. Further, some of the states include assault (non-physical) in their legislation, while others only include battery (physical). The disparity among the 21 states that express legal definitions for referee abuse highlight the inconsistencies in conceptualizing referee abuse from state to state. Further, the law book issued by World Rugby, which details the rules for the sport of rugby, provide no definition of verbal abuse nor does it explicitly address referee abuse (World Rugby, 2017). Therefore, it is not surprising that 
coaches within this study found it difficult to define referee abuse and ultimately utilized indicators of abuse in an attempt to delineate their understandings.

Without an unambiguous definition of verbal referee abuse, three indicators of abuse were determined from the coach interviews through analysis: personal commentary, foul language, and intimidation. The three abuse indicators, as established by the rugby coaches, also aligned well with the extant literature. Perhaps universal abuse definitions can be used across sport, even in niche sports like rugby. According to Evans (2009), name-calling was an indicator of verbal abuse, and explicit name-calling might go so far as referring to the referee through the use of expletives. Using a curse word to name-call the referee could be considered both personal commentary and foul language. Beyond Evans' (2009) inclusion of name-calling as an indicator of abuse, the Women's Sports Foundation (WSF) has also noted that swearing at an official would be considered a marker of verbal abuse (WSF, 2016). The clear linkages in the literature corroborate the coaches' perceptions that both personal name-calling and the use of foul language indicate abuse. Thus, these abuse indicators should be utilized by sport management professionals in developing and distributing a definition of referee abuse that applies specifically to the sport of rugby.

In addition, repetition and intimidation also seem to be indicators of abuse. When conceptualizing these two markers, there was disagreement in the existing research as to what type of abuse is indicated, be it verbal or psychological, but it was clear that both repetition and intimidation denote abuse. When defining abuse and harassment, the IOC defined psychological abuse as "a pattern of deliberate, prolonged, repeated non-contact behaviours [sic] within a power differentiated relationship" (Mountjoy et al., 2016, p. 
1021). The definition highlights repetition as an indicator of psychological abuse, corroborating the findings of this study. Further, intimidation or issuing threats has been shown to denote psychological abuse (Stirling \& Kerr, 2008, 2013; WSF, 2016). While the findings in this study suggest that repetition and intimidation were indicators of verbal abuse, the extant literature categorizes these markers as psychological abuse. The discussion of psychological abuse (or emotional abuse) was almost non-existent within this study; instead, the participants focused on only physical and verbal abuses. Many of the coaches initially suggested that there were limited occurrences of abuse in rugby; however, after a discussion of the behaviors that defined abuses, these perceptions seemed to change. Defining referee abuse helped to clarify the coaches' understanding of the nature of abuse and reaffirmed the importance of defining referee abuse within the rugby sporting structure.

When discussing the prevalence of referee abuse, the Division I-A rugby coaches perceived verbal abuse to be frequently occurring and much more common than physical abuse. As noted in the literature review, referee abuse is a regular occurrence within nearly all sectors of sport (e.g., Dell et al., 2016; Rayner et al., 2016; Walters et al., 2016). The perceived pervasiveness of verbal abuse reported in this study mirrored a 2016 study completed by Rayner et al. where the rugby referees surveyed described verbal abuse to be the most common type of abuse, accounting for $88.8 \%$ of abuses in rugby. All 15 coaches in this study suggested that verbal referee abuse accounted for the majority of abuses that they had witnessed, noting that it was commonplace across various sectors of the sport and more frequent at lower levels. As such, it appears that within the sport of rugby, it will be important to prioritize initiatives aimed at curbing 
verbal abuse. Further, numerous studies have identified abuse as an issue in sport; however, the issue was primarily observed by the victim (the referee). Exposing the ubiquity of referee abuse from an external perspective validates the perceptions presented by referees in previous studies. Here, the coaches, often the perpetrators of referee abuse, called attention to oft-overlooked issues.

The interviewed coaches perceived that abuse behaviors would result in numerous negative outcomes. Negative outcomes suggested included the personal impact on the referee, impact on the match, and impact on the entire rugby community. The three detrimental outcomes of referee abuse were well-documented in the officiating literature as referee abuse has been shown to have an impact on its victims (Fields et al., 2007) and can impact mental health (e.g., Goldsmith \& Williams, 1992; Taylor et al., 1990; Voight, 2009). Further, referee abuse has also been shown to impact the outcome of a game. The coaches noted that oftentimes referees receiving abuse become defensive or retaliatory toward the abusing team. When referees feel vulnerable as the result of abuses, they have been found to react with anger and seek retribution (Lazarus, 1991, 1993). These reactions, then, have the potential to impact the match, as suggested by the Division I-A coaches. In a study of 632 hockey referees, $32 \%$ reported that abuse behaviors directed at them had influenced the outcome of the game (Ackery et al., 2012). Again, there was alignment between the coaches' perceptions of referee abuse and the understandings previously explored from the perspective of the referee.

Further, the coaches believed that referee abuse would influence recruitment and retention of officials. The Division I-A coaches noted that referee abuses would lead referees to reconsider their participation in the sport and may also inhibit future referees 
from establishing themselves in the role. The Division I-A coaches explained that if referees were continually being abused, they may discontinue participation. Referee dropout not only impacts the talent pool but also represents an opportunity cost for the organization (Mountjoy et al., 2016). Opportunity costs for an organization may include the shutdown of leagues as a result of fewer referees, loss of resources invested in training, or loss of organizational knowledge (Dell et al., 2016). The detrimental outcomes of referee abuse, including personal impacts to the referee, match outcomes, and organizational recruitment and retention issues previously presented in the literature seemed to corroborate the coaches' experiences with referee abuse in rugby. The coaches also noted that programs seeking to curb referee abuse must explain the importance of such a cause. Because the coaches were able to identify numerous negative outcomes of referee abuse, these outcomes may help to answer this question: "Why should we make the reduction of referee abuse a priority in rugby?"

When considering referee abuse, the coaches perceived referee abuse, specifically verbal abuse, to be difficult to define, regularly occurring, and the cause of numerous outcomes that could be damaging to the sport of rugby. The coaches' understandings of referee abuse aligned well with the existing literature, which had previously explored abuses in other contexts and from the perspective of other stakeholders. By exploring referee abuse through the lens of the coach, this research has corroborated the findings of studies conducted from the perspective of the referee. This study reaffirms the importance of considering referee abuse as a primary issue in the sport of rugby. Beyond their overall awareness of referee abuse, the coaches noted numerous factors that influenced their perceptions of abuse and their likelihood to engage in abuse behaviors. 


\section{Factors Influencing Referee Abuse}

The factors that influence referee abuse are among the most salient findings from this study. Previous research on referee abuse has explained abuses as the result of overvaluing sport in small communities (Friman et al., 2004), lack of understanding of the rules (Neave \& Wolfson, 2003), and frustration over referee decisions and mistakes (Duxbury, 2002; Friman et al., 2004; Simmons, 2006). These explanations for referee abuse were founded on the presuppositions of the referee and explored single factors that may cause abuses. When considering other types of abuse within sport, many additional studies have noted that there is no single risk factor that serves as the catalyst for abuse (Cicchetti \& Lynch, 1993; Finkelhor \& Baron, 1986; Stirling \& Kerr, 2014; Whittle, Hamilton-Giachritsis, Beech, \& Collings, 2013). The authors of this study contend that referee abuse is no different. There are numerous factors that may influence referee abuse perceptions and behavior. Thus, the current research study was the first of its kind to explore factors that may influence referee abuse perceptions and behaviors from the perspective of a non-referee. The coaches interviewed in this study identified five factors that impacted engagement in referee abuse: (1) personal characteristics/philosophies, (2) relationships, (3) social influences, (4) organizational expectations, and (5) culture.

\section{Personal Characteristics and Philosophies}

Brackenridge (1997a) noted that child maltreatment behaviors were collectively influenced by the perpetrator's personality and contextual dimensions. Consistent with the literature in other contexts, the coaches in this study noted that one's personality had the potential to play a large role in one's perception of referee abuse or the likelihood to engage in abuse behaviors. Ego was a characteristic that seemed to be highly related to 
one's referee abuse perceptions. As the coaches prioritized themselves within the sporting structure, they subsequently devalued other sporting constituents, including the referee. Placing themselves in a position of power seemed to entitle egotistical coaches to engage in referee abuses. Though she did not specify one's egotistical nature as a factor influencing behavior, Brackenridge (1997a) identified high reputation and strong qualifications as factors that may influence maltreatment behaviors in sport. These factors may be closely related to ego. Therefore, perceived qualifications may lead to one believing that one knows more than the referee, and one's reputation for success within the sporting community may also support a high self-regard.

Brackenridge (1997a) also found that in other settings, a previous record of abuse was a factor that influenced maltreatment in sport. Contrary to this finding, the coaches interviewed noted that though many of them had a track record of previous referee abuse, they had matured and developed as coaches, ultimately reimagining their positions on referee abuse and their likelihood to engage in abuse behaviors. As they had matured in their coaching roles, they had been able to disconnect themselves from the player mentality. Maturation increasingly prevented the coaches from being personally impacted by the calls a referee makes, meaning that the mental distance between the coach and the action on the field allowed many coaches to disassociate from the aggressive reactions of their past. In the case of referee abuse, one's personal disposition or maturation level may positively or negatively impact one's abuse perceptions.

Previous scholars have also found that coaches' philosophies were instrumental in influencing the behaviors of themselves and their players (e.g., Martens et al., 2006; Mastroleo et al., 2012). One's coaching philosophy can help to facilitate self- 
development and alignment with that philosophy. Beyond this, the coaches also reported that those who valued player development over winning were less likely to engage in abuse behaviors. Previous research asserts that a sole focus on winning can result in moral behaviors being put aside (Woods, 2007). When athletes overemphasize winning or beating the competition and neglect to focus on personal improvement or performance, they are more likely to perceive cheating and intentionally injurious acts to be acceptable (Duda, Olson, \& Templin, 1991). Stirling and Kerr (2014) found that from the athlete's perspective, emotional abuse was related to a coach's philosophy of athlete development. In this way, the coaches saw emotional abuse as part of the coaching process-a requisite for high-performance success. In the officiating context, the same notion appeared to hold true. If coaches prioritize high-performance successes, they may not only emotionally abuse their players but also the referee as they seek the ultimate goal of winning. In the context of rugby, a coach's philosophy, personal development, and personal characteristics seem to influence referee abuse.

\section{Relationships}

Beyond one's personal characteristics and philosophies, contextual factors within one's environment also influenced perceptions of referee abuse. According to the coaches interviewed, relationships with the referee were seen to reduce abuse behaviors, while a lack of such relationships created a situation where "verbal abuse and physical confrontation is no longer seen to affect a person, but rather, an entity" (Chiafullo, 1998, p. 214). A lack of relationships between the referee and coaches can be related to the social categorization of these roles. When seeking to understand their place in sport, the referee and the coach were able to define themselves and others within their own 
environments (Tajfel \& Turner, 1985). The socially created categories resulted in an ingroup and an out-group as individuals sought to position themselves within their environment and align themselves with others who share their own common characteristics (e.g., Ashforth \& Mael, 1989; Tajfel, 1979; Tajfel \& Turner, 1985). The coaches identified with one another based on their coaching backgrounds and formed a group. Any individuals who did not belong in the coaching group, such as the referees, were assigned out-group status. Once two distinct groups had been created, intergroup comparisons occurred (Tajfel \& Turner, 2001). The coaches referred to this as an $u s$ versus them mentality. Here, the coach and the referee groups were seen as distinct entities, and referee abuse was perceived to increase in accordance with the notion of outgroup dissonance. As a result, abuses perpetrated against referees (i.e., them) would be seen as more acceptable than abuses perpetrated against coaches (i.e., us). Scholars of the sport of soccer showed how this in-group/out-group mentality could impact one's perceptions. Here, soccer fans reported feeling empathetic when individuals on their own team were harmed but felt pleasure when individuals from the other team were harmed. Because the other team was not part of their in-group, the fans' perceptions of morality changed (Cikara, Bruneau, Van Bavel, \& Saxe, 2014). The example of social comparison highlights the ways in which humans create in- and out-groups and how these mental categorizations can change one's perceptions. In the case of referee abuse, a lack of relationships with referees could result in an in- or out-group mentality between referees and coaches. Intergroup comparisons then may result in coaches perceiving that referee abuse was acceptable against referees, though they would not find the same behaviors acceptable if perpetrated against their coaching peers. 
Though a lack of relationships can negatively influence referee abuse, developing relationships between referees and coaches can help to overcome the aforementioned $u s$ versus them mentality. The coaches perceived that when coaches and referees were able to develop relationships with one another, they were able to humanize the individual and bridge the divide between their social categorizations. Building relationships between the two groups could create a foundation for understanding and mutual respect. The coaches explained that the development of relationships would make referee abuse seem more egregious. One coach noted that they had previously told their players not to engage in referee abuse because they knew the referee. Another coach suggested that referee abuse was not worth risking the relationship with a referee. Still another found that their relationship with the referee allowed them to have a conversation with the referee regarding their expectations and frustrations rather than immediately engaging in referee abuse. In each of these instances, the relationship between the coach and referee appeared to be a factor that prevented abuses.

Beyond the relational interactions between the coach and the referee, other social influences were seen to play a role in one's perception of referee abuse. The coaches consistently emphasized the way in which their role with their players reduced their likelihood to engage in referee abuse. Coaches have often been viewed as role models in sport (Kassing \& Barber, 2007; Raakman et al., 2010), which results in their every behavior being watched and, in many cases, imitated by their players (Arthur-Banning, Wells, Baker, \& Hegreness, 2009; Husman \& Silva, 1984; Silva \& Weinberg, 1984). The coaches in this study suggested that while certain characteristics and philosophies may predispose an individual to referee abuse, abusing a referee was primarily a learned 
behavior. The coaches noted that it was their responsibility to deter abuse behaviors in their players and suggested that coaches who did not curb abuses from their players created an environment in which abuse was tolerated. The notion of acceptance of learned behaviors within the sporting environment is supported by Bandura (1973). As the individual interacts with his or her environment over time, he or she learns the behaviors considered acceptable within that environment (Bandura, 1973). Thus, if a coach engaged in referee abuse, the players may see this behavior as acceptable and in the future be more likely to emulate the behavior. The coaches often referred to the behaviors learned from the social environment as a "domino effect" or a "mob mentality." The behavior of a role model or the social expectations set by a coach can influence one individual who may then influence another. Not only does the coach's position of power influence the players' perceptions of abuse, but it also influences his or her own. The coaches in this study reported that they were cognizant of their own sideline behaviors as a result of their players' imitation. In an effort to reduce their players' abuse behaviors, the coaches attempted to mitigate their own participation in referee abuse. The coaches also suggested that coaches and players coming from other sports had learned the acceptable behaviors in those sports, inclusive of referee abuse. When they transitioned into the sport of rugby, they brought with them the learned behaviors from other sports. Because referee abuse was believed to be a learned behavior, that also implies that coaches and sport managers alike have the opportunity to reteach these behaviors and help sport participants relearn the acceptable behaviors in the sport of rugby. This is critical when developing programming that may help to deter abuses. 


\section{Peer Influence}

The coaches reported that peer influence was instrumental in developing their perceptions of referee abuse in rugby. These findings extended the previous literature regarding peer influence, applying existing perspectives to referee abuse. In many instances, the existing literature examined the impact of peer influence on risky decisionmaking and deviant behaviors. As shown by Gardner and Steinberg (2005), when individuals are with their peers, they view risky behaviors more positively and ultimately may take more risks. The relationship between these risk evaluations and behaviors could be associated with one's perception of abuse and subsequent abuse behaviors. Thus, referee abuse (i.e., a negative behavior) may be viewed more positively if the perpetrator were surrounded by a peer group, suggesting that sporting stakeholders could act in ways that are deemed acceptable by their peers. If the peer group supports deviant behaviors, the deviant behaviors of the individual were likely to increase (Dodge, Dishion, \& Lansford, 2007). Deviant behaviors are described as those that violate social norms (Humphrey \& Palmer, 2013). According to the coaches in this study, referee abuse should not be tolerated within the sport of rugby and would thus violate the norms of the sport and be considered deviant behavior. Referee abuse, like other deviant behaviors, is strongly influenced and reinforced by a peer group. The coaches noted that their players strongly influenced the behavior of other players in terms of referee abuse. They suggested that if one player began abusing the referee, it would only be moments until the next player started. Ultimately, peer influence of these players on one another would create a domino effect where the entire sideline could then be seen to engage in deviant behaviors. The coaches also suggested that they had seen the impact of peer influence on 
abuse perceptions and behaviors with spectators. As a single spectator began heckling the referee, it set the precedent that referee abuse was acceptable. Then, another fan could begin abusing the referee, and soon a mob mentality could take over the crowd. No longer was the crowd made up of individuals heckling the referee; they became one unit joined in their disdain for the official.

When the coaches considered their own peer influences, they found that in many cases, their peers positively impacted their perceptions of referee abuse. As previously mentioned, the Division I-A coaches perceived referee abuse to be unacceptable, thus creating a unified, if not formalized, vision regarding referee abuse. Therefore, when a coach participates in referee abuse as part of the Division I-A competition, that coach is looked on as an outsider and the behavior is not reinforced by the peer group. In fact, multiple coaches mentioned that their peers have helped them to reevaluate their own abuse behaviors and reconsider the ways in which they perceived referee abuse. Simply addressing the issue with one's peer coaches seems to go a long way in changing an individual's mentality about referee abuse. If the abusing coach is open to receiving feedback and willing to be self-reflective, peer intervention can be an influential means to change abuse perceptions and therefore behaviors. At this time, much of the peer influence literature suggests a negative influence on the individual, inciting them to participate in behaviors such as drinking, smoking, and drug use (e.g., Go, Green, Kennedy, Pollard, \& Tucker, 2010; Trucco, Colder, \& Wieczorek, 2011; Vitaro, Brendgen, Ladouceur, \& Tremblay, 2001) and reinforcing such behaviors. There are scholars, however, who suggest that peer influence can be positive. In the case of college attendance decisions, peer influence was found to be the best predictor for enrollment 
(Sokatch, 2006); peers were found to influence motivation in sport (Keegan, Harwood, Spray, \& Lavallee, 2009); and peers were seen to be critical in promoting healthy eating and physical activity (Salvy, de la Haye, Bowker, \& Hermans, 2012). In these examples, peer influence was able to promote positive behaviors, just as the coaches have suggested their peers have done in the sport of rugby. Social influences such as personal coaching philosophies and relationships with referees have the potential to influence perceptions of referee abuse in a positive or negative way and help to create a holistic picture of the numerous variables and factors that influence referee abuse perceptions and behaviors. Additionally, the influence of one's peer group seemed to be dependent on the socially accepted behaviors within that peer group; in this way, we see personal philosophies return as a catalyst for peer behavioral expectations.

\section{Organizational Role Expectations}

Beyond the aforementioned factors influencing referee abuse, the Division I-A coaches also found that organizational expectations impacted their perceptions of abuse. For the coaches, the organization set role expectations for the referee - to be fair and maintain a safe playing environment — and when these expectations were unmet, the coaches were more likely to perceive referee abuse to be acceptable. Previous literature has addressed numerous role expectations for the referee, including impartiality (Weinberg \& Richardson, 1990) and the maintenance of a safe sporting environment through rule enforcement and the promotion of safe play (Collins et al., 2008). These role expectations of the referee are well-documented in the literature, and the expectation for their application to the sport of rugby is well understood. Beginning with the expectation for fairness, the coaches seemed to prioritize procedural justice (the belief that the 
process for coming to the decision was fair) over distributive justice, or the ultimate outcome (Chory-Assad \& Paulsel, 2004). The coaches noted that if the referee was performing poorly for both teams, they would have no problems; however, if the referee was perceived to be making one-sided calls or calls that were perceived to be biased against one team, the lack of procedural justice would influence referee abuse. Simmons (2006) suggested that if the concepts of procedural and distributive justice are applied to football, "Players will be more accepting of decisions against them and their team if they perceive that the decision is made in accordance with the rules and that the rules were being consistently applied" (p. 2). When an individual perceives that the rules have not been applied fairly, a coach is more likely to become aggressive (Brockner \& Wiesenfeld, 1996) as the individual is seeking to hold someone (the referee) accountable for the unfair treatment (Greenberg \& Cropanzano, 2001). Because the coaches perceived fairness to be an influential factor in referee abuse, perceptions of organizational justice are noted as a salient finding.

Though previous literature has addressed safety as one of the role requirements of the referee, safety has not been specifically addressed as a factor that influences referee abuse. In fact, in many cases, fairness alone has been noted as a rationale for abuse behaviors. For rugby, the official's role in ensuring safety may go beyond the adherence to regulations. There are numerous situations in the sport of rugby where a team may be participating within the rules of the game but have created an unsafe environment. One such example would be that of a team that is dominant in the scrum. Its dominance results in an unsafe environment for the other team, though they are doing nothing that would be considered illegal based on the laws of the game. In this situation, the requisite 
for a safe environment extends beyond the necessity of making a correct call, and the Division I-A coaches prioritized safety over every other officiating duty. The expectation in the case of the uneven scrum would be that the referee would move to uncontested scrums. The rule change would limit the risk of injury for both teams. Failure to protect the players through adherence to the rules or using inappropriate judgment was seen to influence the coaches' perceptions of referee abuse and the likelihood to engage in abuse behaviors. When considering safety, the coaches often stressed that their primary goal was protecting their players. When the referee failed to ensure the safety of their players, referee abuse was more likely to occur. Within the context of the game, safety played a significant role in influencing a coach's perception of referee abuse. Some coaches went so far as to say that safety concerns were the one reason that referee abuse would become warranted. Though there has been little research regarding the relationship of safety in sport to referee abuse, this seemed to be a salient finding, which suggests that the referee's requirement to ensure safety was a factor that should be considered beyond the general administration of laws within the sport of rugby and that it has considerable influence on referee abuse behaviors.

The organizational role requirements of the referee were understood to include the maintenance of a safe and fair playing environment. When those expectations were upheld, the coaches perceived the referee to be competent, and referee abuse was perceived to be unacceptable and unnecessary. If these expectations were unmet, the Division I-A rugby coaches perceived the referee to be incompetent or biased and at times sought to intervene through abuse of the referee. As such, the coach's 
understanding of the referee's role requirement was influential in their perception of referee abuse and the likelihood to engage in abuse behaviors.

\section{Culture}

Culture was the final factor the Division I-A coaches noted to be instrumental in one's perception of referee abuse. Culture is described as the pattern of values, traits, or behaviors shared by the people in a region (Adler, Doktor, \& Redding, 1986; Hofstede, 1980; Schneider, 1989). When referencing culture, the coaches in this study noted three distinct yet interrelated sporting cultures. They highlighted the American sporting culture, the international rugby culture, and the soccer culture. The American sporting culture was suggested to emphasize winning and normalize abuse behaviors against the referee. These understandings of American sporting culture align with those put forth by Fields and colleagues (2007) who suggested that sporting culture in the United States promotes the importance of sport to such an extent that a win-at-all-costs environment is inevitable. They also found that American sporting culture normalizes violence within sport. "In this [way], each sport has allowed certain actions, such as yelling at the umpire or arguing with the referee, to become traditional conduct which is tolerated and even expected" (Nelson, 1980, p. 2). The rugby coaches suggested that the American sporting norms were influential in impacting abuse perceptions, especially for those new to the sport of rugby. Coaches and players transitioning into rugby from other American sports had grown up with these cultural expectations and understand abuse of officials to be socially acceptable (Kellett \& Shilbury, 2007). As such, they were more likely to engage in referee abuse and to perceive referee abuse to be an acceptable part of sport. 
Much like the American sporting culture, the coaches interviewed suggested that soccer culture tolerated and even promoted referee abuse. In many instances, the coaches noted that in the sport of soccer, the players would approach the referee with their hands in the air yelling and screaming if a questionable call was made. Soccer was associated with hooligans who would attack spectators and venerate violence (Armstrong, 1999). For the coaches, rugby culture was meant to be the antithesis of soccer culture. Those who embodied the ideals of soccer culture would perceive referee abuse to be acceptable and would be likely to engage in abuse behaviors against a referee. So while the coaches found soccer culture to negatively influence referee abuse in other sports, in regard to rugby they utilized soccer mainly as a means of comparison. Beginning when William Webb Ellis picked up the soccer ball and ran with it (Collins, 2009), the disassociation of rugby from the sport of soccer was of primary importance to the rugby coaches.

The coaches felt that rugby culture was distinct from American sporting culture. Unlike the American sporting culture where referee abuses were tolerated and, in some instances, praised, rugby culture (or international rugby culture) was seen as a game still governed by core values, including integrity, passion, solidarity, discipline, and respect, as outlined by the World Governing Body for Rugby (World Rugby, 2014). Rugby culture suggested that playing rugby was an ode to moral welfare (Hughes, 1895) and that the game was a gentleman's sport founded on respect. The historical underpinnings of the sport signify that the participants may still engage in rugby competition with a high level of civility (Moore, 2000). These notions were underscored by the Division I-A coaches who suggested that respect was a key value that should be upheld in the sport and that respect should be extended to all participants. As respect was expected to be 
extended to all participants, those immersed in the rugby culture perceive referee abuse to be unbecoming and a poor reflection of the sport of rugby. For many of the coaches, the immersion in rugby culture created a requisite standard of behavior and differentiated the sport of rugby from other American sports. The discussion of culture highlights the importance of considering referee abuse holistically. While many coaches perceived themselves to be immersed in rugby culture, a number also suggested that they had engaged in referee abuse or found themselves participating in abuses more frequently in America than they had in other countries. That suggests that rugby culture may lay a foundation that helps to detract from abuses, but that multiple factors may coalesce to influence one's perception of abuse.

Interestingly, there was not a marked difference between those who had grown up in the United States versus those who had grown up abroad when it comes to understandings of rugby. It seems that the majority of American-born coaches interviewed had either lived or spent significant amounts of time traveling abroad. As such, they had been exposed to international rugby culture even though they may have been raised in an American sporting culture. Therefore, the acceptance of cultural norms was not a result of one's nationality alone. In this case, the question remains: Why have the Division I-A coaches chosen to prioritize their understandings of rugby culture over the cultural norms and expectations of American sporting culture? Answering this question may help to develop programs that foster the growth of rugby culture for those new to the sport.

In addition to one's culture, referee abuse in rugby was perceived to be influenced by four other factors: (1) personal characteristics and philosophies, (2) relationships, (3) 
peer influences, and (4) organizational expectations. Despite the distinctiveness of each of these factors, it is clear that these factors may also influence one another. For example, one's culture was likely to influence one's personal philosophies. As such, these factors can be understood both individually and collectively as influencers of referee abuse.

Further, variations of each factor have the potential to influence referee abuse in a myriad of ways. As discussed, one's adherence to the social norms of American sporting culture may lead to the acceptance of and participation in referee abuse, while those immersed in rugby culture find referee abuse to be abhorrent. Thus, the influence of the factor on referee abuse is clear, though the outcome of the influence may be variable.

\section{Solutions}

Considering referee abuse through the lens of the coach creates a unique opportunity to explore coach-derived solutions. As potential perpetrators of referee abuse, coaches offer a unique perspective on referee abuse. Coaches hold a powerful position within the sporting structure (e.g., Martens et al., 2006; Mastroleo et al., 2012; Perna et al., 1996); thus, solutions put forth are uniquely poised to generate buy-in from players and administrators, which could ultimately support referees in their quest to mitigate referee abuse.

\section{Comprehensive Approach}

When discussing potential solutions for referee abuse, the coaches noted that the approach must involve all rugby sporting stakeholders, which aligns with Brackenridge (1997b) who noted, when considering sexual abuse in sport:

A systems approach to sexual abuse is important if we are to address the cultural and structural changes needed to prevent it. This requires all stakeholders to recognize that they have responsibilities within the system and to overcome their tendencies to deny the reality of the issue. (p. 115) 
To reduce tolerance and prevent abuses, a systematic multi-agency approach is found to be most effective (Brackenridge, Fasting, Kirby, \& Leahy, 2010). The use of multiple interventions and addressing the problem in multiple settings can help to create uniform messaging across a sport. In this way, referee abuse should be addressed within the USA Rugby organizational structure, within the league or conference, and within the individual team.

\section{Proactivity}

Beyond the use of a comprehensive approach, the coaches also suggested that programming should be proactively executed rather than waiting for serious issues to arise. Intervention programs often start as the result of high-profile cases of abuse (Kerr, Stirling, \& MacPherson, 2014). The current lack of proactivity suggested that practitioners may feel impotent in affecting change (Stirling \& Kerr, 2008) or may not know how to approach such issues. Thus, proactive research and the implementation of solutions generated becomes all the more valuable. The coaches' position of power within the sporting structure could be utilized to eliminate resistance and solicit buy-in from additional stakeholder groups. Because the coaches have developed the solutions discussed within the findings, they also stand to serve as advocates for change. Beyond the belief in comprehensive and proactive programming, the coaches also specified three strategies to help reduce referee abuse: implementation of policies and sanctions, education, and collaboration.

\section{Policies and Sanctions}

The coaches suggested that effective policies and protocols must be in place and regularly enforced. The first step was enhanced awareness of referee abuse policies and 
protocols. Many of the coaches noted that while they knew sanctions existed for referee abuse, they were unsure of what those sanctions may be. As a result, the coaches suggested that a clear definition of referee abuse be identified, which should include examples of various abuse behaviors. Further, they noted that even when they had witnessed referee abuse, there were rarely any known repercussions for such actions. Cleland and colleagues (2015) noted that inconsistency in applying policy undermines the value of the policy in changing behaviors. To combat this problem, the coaches believed that referee abuse should be addressed in a consistent manner. Expectancies of punishment for aggressive acts could be learned (Tenenbaum, Singer, Stewart, \& Duda, 1997), so when individuals witnessed referee abuse going unpunished, they were more likely to consider that behavior acceptable. Conversely, the individuals would be less likely to participate in referee abuse if they had seen another individual punished for such behavior (Husman \& Silva, 1984). When abuses were witnessed, the coaches believed they should be reported, and the perpetrator should be appropriately sanctioned by the league or USA Rugby. The coaches suggested that if consequences for referee abuse were seen, that would deter abuse behaviors and set the precedent that referee abuse would not be tolerated in rugby. Previous research has also suggested that increasing punitive or disciplinary measures may serve as an external inhibitor. In this way, the policies could serve as a barrier to abuse (Finkelhor, 1984). The International Society of Sport Psychology (ISSP) recommended that in order to deter aggressive behavior, "management should make fundamental penalty revisions so that rule-violating behavior results in punishments that have greater punitive value than potential reinforcement" 
(Tenenbaum et al., 1997, p. 5). As such, the requisite for adequate sanctions and the uniform enforcement of regulations becomes critical in curbing referee abuse.

\section{Education}

"Education should be the cornerstone of any safe sport strategy" (Mountjoy et al., 2016, p. 1025). The coaches agreed with this notion by suggesting that additional education was necessary for numerous parties in order to help curb referee abuse. The first group to be considered for additional educational training was the referees. The coaches suggested that continuing education for rugby referees was necessary. The lack of continuing education had previously been noted by Simmons (2006) who found that referees themselves report few specific opportunities for continual learning. As a result, referees often felt unprepared for dealing with abuses (Simmons, 2006). Instead of training referees solely on rule enforcement, the coaches also believed that training should focus on the interpersonal skills necessary to be successful in the officiating role. Coaches suggested that referee training should require a weekly component, noting that coaches and players practice multiple times a week in preparation for their games.

In addition to referee education, education for coaches and spectators was also suggested. The coaches felt that coaching certification should include information about referee abuse to increase awareness of the issues. Further, these trainings should specify the impact of referee abuse on the sport. As the coaches have explained, acknowledging a problem is the first step, but explaining the impact of that problem as it relates to the coach can help the coaches understand the relevance of the issue. The ISSP agreed, stating, "Coaches should be made aware of the potential damage of aggression in sport, not only for their team and the player at hand, but for society as a whole" (Tenenbaum et 
al., 1997, p. 4). Further framing the impact of referee abuse, the coaches believed, could contextualize the issue for the coach and thus help to influence perceptions of abuse. The coaches also suggested that coaching certification should require one to walk in the referee's shoes, thus becoming a certified referee. Serving in an officiating role could help coaches to humanize the position of a referee and address the in- and out-group issues mentioned in a previous section (Tajfel, 1979). If coaches become certified referees, they are now part of the same group as the referees. In this way, the stereotypes that may have once applied to referees and allowed for referee abuse now apply to the coaches as well. As a result, the coach and referee occupy the same sporting space, and the coach may be less likely to perceive the referee as an outsider or see abuses against the referee as tolerable.

Finally, the coaches suggested that spectators should be educated about the laws of the game and the rugby culture. The coaches believed that the majority of rugby fans were not familiar with the laws of the game and did not have a rugby background. Thus, educating spectators about the rules of the sport may serve to reduce abuses as spectators would then be less likely to heckle a referee as a result of their own naivety. Further, the coaches noted that educating spectators about the rugby culture could set the precedent that the referee was to be respected. Through cultural education of the spectator, the coaches would have more power to intervene when abuse behaviors did exist because the behavioral standards for the sport had been set. Additional and ongoing education was believed to be of utmost importance in combating referee abuse. It provides an opportunity to engage all of the stakeholders in a common vision and to combat referee abuse through a myriad of constituency groups. The value of engaging all stakeholders to 
combat referee abuse was again highlighted as a strategy when the coaches considered the importance of collaboration.

\section{Collaboration}

Collaboration was the final solution put forth by the Division I-A coaches. They noted that in many instances, coaches and referees operate within their own silos, which only served to increase the divide between the two groups. As previously mentioned, the coaches felt that a lack of relationship was a factor that could influence referee abuse, so the solution for overcoming the issue would be working together to develop relationships between the two groups. In previous research on officiating, many referees have suggested that they have discontinued officiating due to a lack of referee community (Kellett \& Shilbury, 2007; Kellett \& Warner, 2011). The authors of this research also proposed that a referee's community has helped them learn how to deal with abuses (Kellett \& Shilbury, 2007; Kellett \& Warner, 2011), thus helping referees to reframe the abuse they received, which enabled them to continue in the role. Perhaps, based on the coaches' proposed solution for referee abuse, the lack of community that referees experience extends beyond their own role group to include a lack of relationships with coaches and admittance into the larger rugby community. To facilitate the development of relationships between these two groups, coaches proposed joint trainings, meetings, and after-match functions. Each of these scenarios present an opportunity for coaches and referees to interact outside of the match. In this way, referees and coaches build relationships and begin to think of one another as part of the same group, working toward the same goals. Collaboration can help to eliminate social comparison and favoritism resulting from the in- and out-group mentality (Turner, Brown, \& Tajfel, 1979) between 
coaches and referees, and ultimately help reduce abuses. For coaches, the priority in these collaborative activities was not to increase one's knowledge of the game or understanding of the laws, but rather the value comes in the shared experience between two individuals. This aligns with previous research that noted that social experiences beyond the field are important to an official's continuation (Kellett \& Shilbury, 2007; Kellett \& Warner, 2011). The proposed social experiences may help to deter abuse, which may ultimately prevent referee dropout. The goal of these social interactions was learning more about a referee or a coach, understanding where they come from and why they serve in the role, and ultimately respecting them as another participant in the game. The dissolution of the idea that the referee role was secondary to other sporting roles and that referees ought to remain separate from their sporting counterparts allows for the development of relationships. According to the Division I-A coaches, these relationships were the foundation for bridging the divide between the two groups, eliminating the $u s v s$. them mentality and deterring referee abuse.

Collaboration also provided coaches and referees with an opportunity to share opinions and feedback with one another. In many instances, the desire to speak up arises when there is a perceived need for improvement (Hirschman, 1970), an example being a coach desiring to express growth points to a referee. In many instances, being heard may deescalate a situation. Thus, providing opportunities for referees and coaches to engage in constructive conversations may reduce future abuse behaviors. Social interactions between coaches and referees could provide an opportunity for dialogue and help both groups to understand the rationale for certain decisions. As with other organizational contexts, when an individual "feels heard, they may experience positive 'expressive' or 
attitudinal outcomes such as an enhanced sense of justice, motivation, or satisfaction" (Burris, Detert, \& Romney, 2013, p. 22). In the coach's perspective, the ability to provide feedback and be heard could then reduce instances of abuse. That aligns with previous research findings since being heard leads to an enhanced sense of justice. When the coaches perceive that a referee is being just, they would be less likely to engage in abuses.

The solutions for deterring referee abuse put forth by the Division I-A coaches closely aligned with previous research, and the existing literature supports their belief that these solutions could serve to reduce referee abuse. Taking a proactive stance against referee abuse, engaging all stakeholders in the process, enhancing policies and enforcing protocols, developing a more robust educational platform, and providing opportunities for collaboration come together to create a comprehensive plan for deterring referee abuse in rugby.

\section{Proposed Model}

The confluence of effects from culture, organization, relationships, and personal characteristics come together to influence one's perception of referee abuse and, ultimately, the solutions that one may propose regarding abuses. Individuals exist within a larger ecology and are unable to disassociate themselves from the external influences that exist in their lives. According to the coaches in this study, the same appears to hold true in regard to referee abuse. In considering the factors that influence referee abuse, the coaches highlighted numerous personal and environmental influences. Upon comparing the emergent themes with existing literature, the ecological-transactional framework best described the coaches' experience with referee abuse. This framework has previously 
been utilized to assess topics closely related to referee abuse, including child maltreatment (e.g., Cicchetti \& Lynch, 1993) and emotional abuse in the coach-athlete relationship (e.g., Stirling \& Kerr, 2014). In both examples, the ecological framework assessed vulnerabilities, and variables of risk were organized into the following four categories: (1) ontological development (personal characteristics and philosophies), (2) the microsystem (relationships/social influences), (3) the exosystem (organizational expectations), and (4) the macrosystem (culture). The emergent model, which reflects themes derived from the participants in this study, does not consider referee vulnerabilities to abuse. Rather, because the study was conducted from the perspective of the coach, it considers factors that would influence one's perception of referee abuse or impact the likelihood to abuse a referee. 


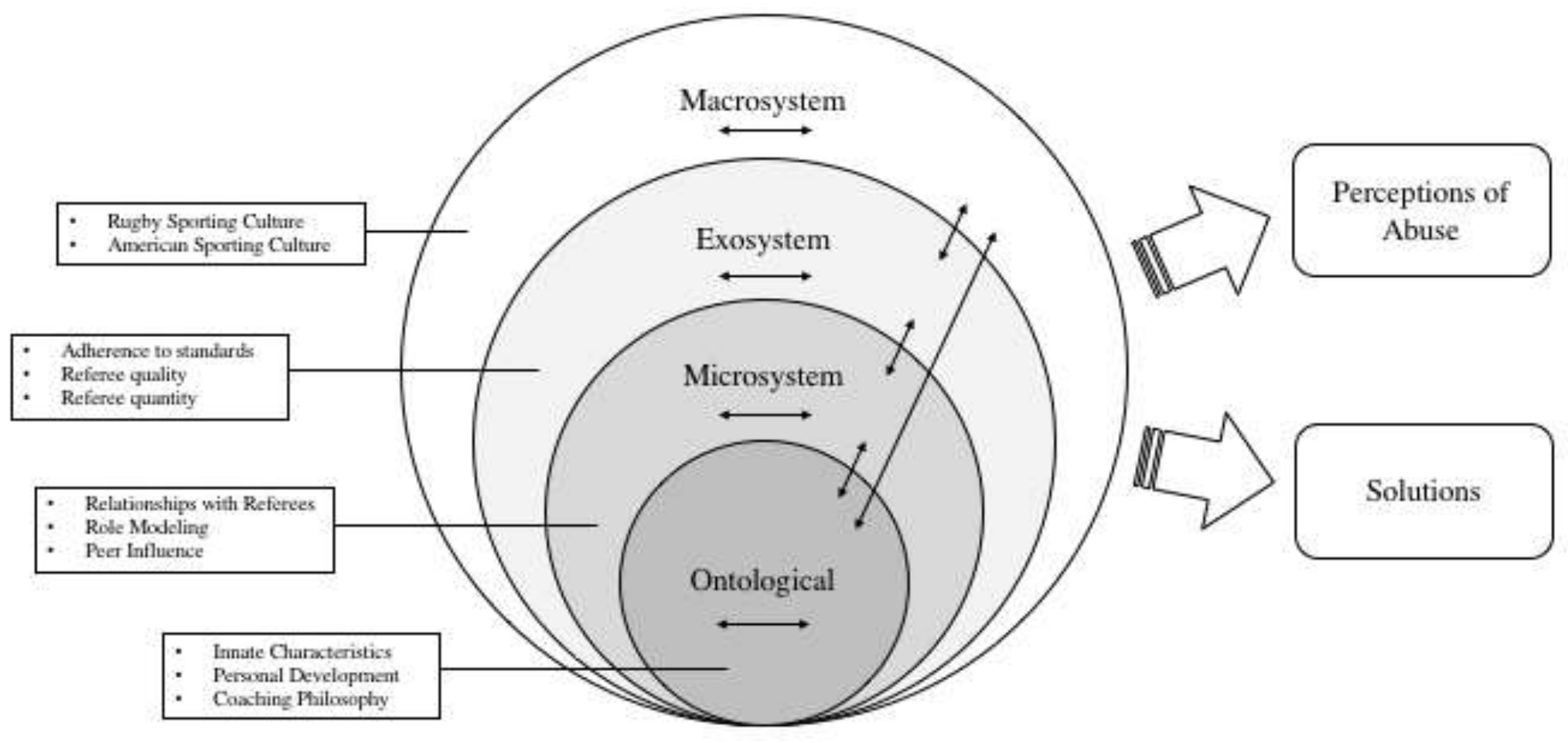

Figure 5. Ecological Transactional Model of Factors Influencing Referee Abuse in Rugby 
The Ecological Transactional Model of Factors Influencing Referee Abuse in Rugby (ETM-FIRAR), shown in Figure 5, divides one's environment into four sectors. Based on the data collected in this study, the four ecological level headings could be described as follows: (1) personal characteristics and philosophies, (2) relationships and social influences, (3) organizational influences, and (4) culture. However, to ensure consistency with the terminology utilized in the existing literature, each of these headings has been relabeled. Thus, the model presents the etiology of referee abuse by organizing variables of influence into the following categories: (1) ontological, (2) microsystem, (3) ecosystem, and (4) macrosystem (Cicchetti \& Lynch, 1993; Stirling \& Kerr, 2014). At each level of ecology, discussed below, variables present may determine perceptions of abuse or one's likelihood to abuse a referee.

\section{Ontological Attributes}

The ontological level of the ETM-FIRAR includes the factors within the individual that could be associated with being a perpetrator of referee abuse (Stirling \& Kerr, 2014). In this instance, such factors may include one's personal characteristics, one's coaching philosophy, or one's own professional development. In each instance, the factor influencing referee abuse is directly tied to the individual. In the case of a coach perpetrating referee abuse, the coach's innate aggressiveness or egotistical nature may play a role. In this way, a coach who tends to react aggressively to conflict may bring these character traits into the understandings and behaviors regarding referee abuse. For example, the coach with an aggressive personality is frustrated by a penalty called against his or her team. The aggressive nature of this coach may lead to vulgarities being yelled 
at the referee. As seen in this example, the coach perceives this aggressive response to be appropriate because this response aligns with his or her behaviors in other contexts. Thus, individuals cannot disassociate from their personal tendencies, and the coaches interviewed believed these personal characteristics could influence referee abuse.

Another such example would be that of personal development. Many of the coaches in this study noted that as young or inexperienced coaches, they were more likely to engage in referee abuse behaviors. As such, one's personal growth and development can be a factor that influences abuse. When individual coaches became more reflective and engaged in both professional and personal development, they seemed to find less value in abusing the referee. Throughout the coaches' maturation process, they noted that referee abuse does not accomplish any positive outcomes and thus was not a valuable practice. We see that the personal development has led to a change in perceptions of referee abuse.

The final personal factor that arose in the findings of this study was one's coaching philosophy. This seemed to result from a unification of personal values or characteristics and one's own personal development. As in other studies, a coach's philosophy is instrumental in influencing behaviors (e.g., Martens et al., 2006; Mastroleo et al., 2012). The coaches perceived the same to hold true regarding referee abuse behaviors. If, within one's coaching philosophy, all participants in the game are to be respected, then it follows that referee abuse should always be considered an affront to that philosophy. As such, the coaching philosophy serves to set a behavioral standard regarding referee abuse and has the potential to influence one's perceptions of abuse. 
When considering these examples, it is important to note that for each positive influence that one of these factors may have, there is also the possibility of a negative influence. For example, if a coach's philosophy is founded on winning at any cost, the well-being of the referee may be seen as inconsequential to the coach. These personal attributes have the potential to influence referee abuse both positively and negatively. Further, there is also the potential for interaction among the factors within each ecological level. Coaches' personal value systems might impact their willingness to pursue personal development, creating the potential for intra-level interactions. These intra-level interactions are depicted by the two-way arrow shown at each ecological level. The ETM-FIRAR proposes that an individual's personal characteristics, personal development, and coaching philosophy are all ontological attributes that influence referee abuse.

\section{Microsystem}

The next level of the Ecological Transactional Model of Factors Influencing Referee Abuse in Rugby, the microsystem, encompasses one's immediate relationships. Referee abuse is shown to be influenced by peers, role modeling relationships, and relationships between the coach and the referee specifically. When considering peer influence as a factor that impacts referee abuse, the people with whom the coach associates may impact the coach's behavior. For example, if a coach is surrounded by a community of fans shouting abuse at the referee, that coach could become associated with this mob mentality and perceive the abuses to be acceptable or rationalized. The opposite may also hold true; if the coach is surrounded by assistant coaches who value and respect the referee as part of the game, the coach may not engage in referee abuse 
behaviors in order to normalize his or her behaviors within the coaching context. Thus, peers can be both a positive or negative influence on abuse behavior.

The coaches in this study also noted that their own role-modeling relationships played a part in the way that they perceived and approached the subject of referee abuse. According to Bandura and Walters (1977), human behavior is learned through modeling; from observing others, one develops a template for behavior and later utilizes this behavioral understanding to guide one's action. In this way, the behaviors of the coaches have the potential to impact the future behavior of their players. The coaches in this study strongly believed that they serve as role models for their players and, aligning with Bandura and Walter's belief, found that their players mirrored their own behaviors. The potential for role modeling behavior influenced these coaches to self-report that they exhibit positive reactions to and relationships with referees. In this way, the coaches sought to prevent their players from modeling negative behavior. Interestingly, their focus on avoiding poor behavior modeling deterred abuse behaviors from the coach.

Finally, the relationship between the coach and the referee was suggested to be an incredibly influential factor when considering referee abuse. Coaches noted that if they had personal relationships with the referees, they were less likely to engage in referee abuse behaviors. Additionally, these relationships helped to create mutual respect between the two parties and allowed for opportunities to air grievances before they led to abuse behaviors. The coaches noted that the development of relationships between referees and coaches would be beneficial in influencing abuse behaviors and changing perceptions of abuse. 


\section{Exosystem}

In this model, the exosystem includes the communities that a rugby coach may operate within (e.g. Belsky, 1980; Cicchetti \& Lynch, 1993; Stirling \& Kerr, 2014). Because this study was limited to one league (Division I-A) within a single sporting entity (USA Rugby), the impact of various communities on a coach's perception of referee abuse is underdeveloped. Within the model, the researcher noted organization as a community that the coaches exist within but had limited data regarding other communities that may also influence abuse. The first factor that was seen to influence abuse at a community level was the lack of adherence to standards. The coaches noted that referee abuse was regularly occurring but that few had seen sanctions enacted for abuses. If there were no detrimental outcomes for the coach who engaged in negative behaviors, there was no reason for the behaviors to be discontinued. The participants in this study also noted that increasing and enacting consequences and standards would send a message through the coaching ranks regarding USA Rugby's stance on referee abuse. Sanctioning coaches who engaged in referee abuse would potentially influence the behaviors of repeat offenders or set an example for other coaches who had been engaging in similar behaviors.

In addition to consequences for engaging in referee abuse, referee quality and quantity were both seen as community level factors that influence abuse. In the coaches' perceptions, USA Rugby should be adequately training officials and providing a team of three to Division I-A matches. Both of these issues suggest that the referee was not important within the sporting structure. Not only are they undertrained, but they are also expected to maintain control of a game with no support. That creates a scenario in which 
coaches felt that their knowledge of the game was superior, which then gave them permission to question and critique the referee. In terms of referee quantity, USA Rugby, or their subsidiaries, were only assigning one referee to a game that should require three officials. In this way, the referee is left with no support and faces a 30-to-1 player-toreferee ratio and a 2-to-1 head-coach-to-referee ratio. In both scenarios, they begin the match outnumbered. The lack of support can create the potential for abuses as coaches are intrinsically provided a position of power. The factors that influence referee abuse at the exosystem level were strongly related to the coaches' expectations of the organization and for their competition. When these expectations were unmet, the potential for referee abuse increased.

\section{Macrosystem}

In the Ecological Transactional Model of Factors Influencing Referee Abuse in Rugby, the final level of consideration is the macrosystem. Within the macrosystem, one's perceptions may be impacted by culture, government, and bodies of knowledge (Stirling \& Kerr, 2014). In the case of referee abuse in rugby, the coach's primary focus was the impact of culture. Though all of the participants interviewed in this study resided in the United States, world cultures and sporting cultures were both noted as factors that influenced referee abuse. As previously mentioned, American sporting culture tends to be one that normalizes and, in some instances, condones abuses (e.g., Kellett \& Shilbury, 2007; Nelson, 1980). In this way, traditional American sports such as football, baseball, and basketball were believed to allow for abuses within the confines of their sporting cultures. The coaches suggested that these American sporting cultures had the potential to negatively influence one's perception of abuse, as abuse behaviors may be considered 
acceptable. It was also suggested that American sporting culture influenced rugby culture as the sport continued to grow. Players from other sports were beginning to pursue rugby, and they were seen to bring their sporting values and norms into the sport. The coaches believed that rugby culture, also referred to as international rugby culture, had the potential to decrease abuse behaviors. Rugby culture was founded on the principle of respect (World Rugby, 2014). In this way, all participants in the game were to be respected, including the referee. As such, the coaches believed that those who had been immersed in rugby culture would perceive referee abuse as unwelcome in the sport. On the other hand, those who had been exposed only to American sporting cultures or international soccer culture would find abuse acceptable, thus perpetuating the belief that referee abuse is a convention of sport. The exposure to and immersion in varying sporting cultures thus had the potential to influence abuse perceptions and, subsequently, abuse behaviors.

\section{Intra-Level and Inter-Level Interaction}

Based on the proposed model, there are numerous factors that may influence referee abuse in rugby, with four categories of ecology that help to organize these factors. The ecological levels do not exist within their own sphere of influence; instead, each level (ontological, microsystem, exosystem, macrosystem) has the potential to impact the other levels. Such an example would include relocation to a different country. In relocating, the individual would be exposed to a new culture and, potentially, new sporting cultures. These new experiences may then impact one's coaching philosophy, and as previously mentioned, one's coaching philosophy then influences one's perception of referee abuse. These inter-level interactions are depicted by the arrows between each 
level of the model. While the model only shows interaction to exist with the next level, the double-sided arrow that travels through each level is meant to show the potential for interaction among any of the levels.

\section{Perceptions of Abuse and Solutions}

Additionally, the large arrows in the diagram, which point from the coach's ecology towards proposed solutions and perceptions of referee abuse, suggest that these ecological factors not only impact one's perception of referee abuse but also the way that they may suggest future change. The aforementioned sections detail the ways in which these factors influence one's perception of referee abuse. In regard to solutions, future solutions proposed by the coaches were often directly related to the various factors within an ecological level. Such an example would be the development of joint education programs between referees and coaches. This proposed solution reaffirmed the lack of relationships with referees as a problem that leads to referee abuse. These perceptions led to the suggestion of a joint educational opportunity as a viable solution to help deter referee abuse. In this way, many of the solutions proposed by the coaches as part of this study sought to counteract the factors that negatively influence referee abuse or to emphasize and prioritize the factors that deter referee abuse.

The Ecological Transactional Model of Factors Influencing Referee Abuse in Rugby is proposed as a means to conceptualize the factors that influence abuse behaviors in the sport. The model extends previous models put forth by Cicchetti and Lynch (1993) and Stirling and Kerr (2014) addressing the etiology of referee abuse. Unlike these previous models, the ETM- FIRAR explores factors that influence the perpetration of abuses, rather than the vulnerability to abuses. As suggested by the Division I-A rugby 
coaches, perceptions of referee abuse and abuse behaviors are strongly influenced by environment. Thus, the model divides environment into four sections in order to better understand each component. The ontological level of the model represents one's personal characteristics, or internal environment. The microsystem explores the relationships of the coach, or their immediate social environment. The exosystem highlights the communities within which the coach exists. Finally, the macrosystem encompasses the cultural understandings of one's world. As previously mentioned, each of these environments has the potential for both intra- and inter-level interaction, where factors existing within each level may influence one another and the levels themselves may follow suit. Because this study is exploratory in nature, this proposed model should be applied to other contexts and stakeholder groups to determine how it functions in other settings.

\section{Practical Implications}

As suggested by the coaches in this study, the prevention of referee abuse requires a holistic approach and the development of interventions that address the etiology of referee abuse at various levels. A proactive approach to referee abuse is important along with the buy-in from various stakeholder groups. As the coaches have mentioned, they do not want to see rugby become a sport like football or soccer where referee abuse is accepted. Additionally, the consensus that referee abuse is impacting referee recruitment and retention emphasizes the issue for the sport. Thus, turning an eye toward referee abuse should become an immediate priority within the sport of rugby. While the coaches have highlighted numerous solutions that should be considered in curbing referee abuse, this section serves to emphasize them. 
The relationships built between referees, peers, and players are incredibly influential. As the coaches mentioned, providing additional opportunities for referees and coaches to interact would be instrumental in curbing referee abuse. Such opportunities may include the reintroduction of after-match functions in areas where these traditions have fallen away. They provides an informal setting in which coaches, referees, and players can address any issues or inconsistencies and come to know one another as individuals. Further, joint education programs would also help to develop the relationships between referees, coaches, and the coach's peer group. In this way, groups would be able to explore the same topic and share their perspectives, ideally creating consistency for all parties involved.

There are several organizational implications that have arisen from this study. The coaches have noted that protecting referees from referee abuse extends beyond the impact on the individual and has the potential to impact the availability of the game. As a result, it is paramount that educational programming not only defines referee abuse and presents sanctions for abuse behaviors but also provides a rationale for the importance of such protocols and consequences. OY mentioned previously that one cannot simply enact new policies but must explain why. These explanations create the foundation for buy-in within various sporting stakeholder communities. Beyond this, policies, procedures, and consequences regarding referee abuse should be created in collaboration with various stakeholder groups. Each group has its own perspective, and this is paramount in generating support for new ideas and regulations. Again, in regard to regulations, administrators and coaches alike must then uphold the regulations put forth. This cannot be an empty promise, rather the standard must be set and followed. Beyond this, a team 
of three referees should be provided for each match. An adequate number of referees at the match provides support for the center referee but also helps to reduce error as there are three sets of eyes viewing every action. Further, continuing education should become a requirement for the referee. It is inadequate for the referee to be provided a lifetime certification for the completion of a single eight-hour course. Additional education requirements may help to enhance referees' knowledge, help them to maintain currency, and counter the argument that referees do not invest enough time in their own training and education. Finally, as was consistently suggested by the coaches in this study, another strategy to curb referee abuse would be to require coaches to be certified as referees as part of their coaching certification. This dual certification would help create empathy between the two parties and would also provide a great opportunity to implement joint education and training. Though not suggested by the participants in this study, it may also be beneficial for referees to become dual certified as coaches, providing the same benefits for referees that referee certification may provide for coaches.

Beyond this, the coaches have highlighted the importance of retaining the rugby culture in the ever-changing sporting environment. The first requirement here is the indoctrination of new participants into the sporting culture. This may mean that coach education could also include information on the rugby culture and values of rugby. It may also require the personal commitment from coaches to uphold certain behavior standards and embody the values of rugby.

Finally, the findings of this study suggest that referee abuse is believed to be a regularly occurring issue in rugby. The coaches in this study and referees in numerous 
previous studies (e.g., Dell et al., 2016; Rayner et al., 2016; Webb, Rayner, \& Thelwell, 2018) concur that referee abuse exists and should be minimized. As this study recommends, considering interventions from the ontological to macro spheres of influence may be effective in curbing abuse behaviors, and this proposed model provides a structure for addressing referee abuse holistically. Working together, sport managers, coaches, and referees may be able to utilize the ecological approach to help deter referee abuse in rugby.

The creation of the Ecological Transactional Model of Factors Influencing Referee Abuse in Rugby creates a foundation from which sport managers can begin to develop programming that seeks to reduce referee abuse. The ETM-FIRAR provides a framework that illuminates numerous factors that influence referee abuse. Thus, new programming can follow this model in order to address various factors that underlie the existence of referee abuse. The reduction of referee abuse is of utmost importance to all sporting stakeholders, and the coaches in this study have confirmed that referee abuse likely leads to recruitment and retention issues for referees. That makes referee abuse a relevant issue for sport managers.

Further, this study presents a myriad of solutions for referee abuse, as articulated by rugby coaches. The development of these solutions from a coach's perspective may help to solicit buy-in from additional coaches. Not only are coaches common perpetrators of referee abuse, but coaches are also incredibly influential within the sporting sphere. Thus, the inclusion of coaches in the development of solutions that may reduce abuses creates a strong foundation for future change. 


\section{Limitations}

Several limitations existed within this research study. As a result of the phenomenological nature of the inquiry, this study sought to explore Division I-A rugby coaches' perceptions of referee abuse within a specific context. Because of this, it is difficult to extend those understandings of referee abuse to other settings. While this study reflects rugby coaches' understandings of referee abuse, these perceptions may be limited to the rugby sporting context. Future extrapolation would require the application of the findings presented in this study to other contexts to help determine the generalizability of these findings.

The study was further limited by the data, which were $100 \%$ self-reported. Because the data in a phenomenological study sought to represent the meanings and experiences of the individual as lived, self-reporting was the primary means for data collection. The difficulty in utilizing self-reported data was that there were no means to independently verify the perceptions put forth. Thus, the data may be biased by the selective memory of the participants or social desirability. Selective memory occurs when participants include and exclude particular memories in their discussions. Selective memory may potentially impact the findings of this study as the individuals may have chosen to include memories that shed a positive light on coaches or devalued the referee.

Further, the inclusion or exclusion of experiences may come from the desire for social desirability. Because abuse of any sort can be considered taboo, it is possible that the coaches interviewed responded to questions regarding referee abuse in a manner that would have been perceived as socially acceptable. Socially acceptable responses may have included the demonization of referee abuses in rugby and the suggestion that abuses 
are occurring but rarely within the DI-A competition. As such, social desirability has the potential to influence the findings of this study. Though there is the potential for both selective memory and social desirability to impact a participant's self-reported data, the researcher completed two interviews with each participant in order to create an open and honest environment. That provided coaches with an opportunity to feel comfortable discussing their true thoughts and feelings regarding referee abuse. Further, many of the coaches discussed their own participation in referee abuse and were honest in reporting their own negative behaviors. Such responses led the researcher to believe that social desirability and selective memory had a limited influence on the outcomes of this study.

Finally, this study was limited by the researcher's perceptions and understandings of the data. As part of a hermeneutic phenomenological study, it is understood that all individuals participating in research have their own understandings of the world, which are influenced by their experiences. These experiences cannot be $100 \%$ eliminated from research; thus, unlike transcendental phenomenology, bracketing out one's experiences is believed to be difficult, if not impossible. Thus, in understanding the existence of my innate biases and experiences, I present myself as a limitation to this study. As the sole researcher for this project, I was able to create, review, eliminate, and create again the various themes and categorizations put forth as a part of this study. In reviewing the data, another researcher may categorize various themes in different ways or identify other literature that may be relatable to the topic of referee abuse. While I believe that the interpretation of the data presented appropriately analyzed the findings, there may be other ways to consider this data as well. To ensure that my own biases have been controlled within this study, I have presented a researcher's stance that acknowledges my 
background in rugby. In addition to the researcher's stance, I have utilized an inductive data analysis method, spending weeks organizing codes into themes, naming themes, reorganizing themes, and renaming themes. Inductive analysis helped to ensure that the data, rather than the researcher's pre-existing understandings, guided the outcomes of the study.

\section{Future Research}

Because this study is exploratory in nature, there are numerous avenues for future research. One minimally discussed yet potentially interesting finding was the potential for generational differences to impact referee abuse. Generational differences among coaches may be related to the chronosystem, which is a component that has been presented in other ecological models. The chronosystem, presented as the fifth level of Bronfenbrenner's (1979) model, includes the socio-historical events that may impact an individual. As such, the generations that coach are born into may impact their perceptions of referee abuse based on their experience of varying socio-historical events. While these ideas are merely conjecture based upon the minimal discussion of coaches in this study, the inquiry into generational differences among coaches warrants further inquiry.

In addition to generational differences, considering one's gender may be valuable in understanding perceptions of referee abuse. In this particular study, there was only one female represented. As such, there was not enough data to compare the perceptions of male and female coaches. When it comes to institutional violence, women are more commonly exposed to such behaviors, and this may impact the way that they understand referee abuse. As a result, understandings of referee abuse may vary by gender. 
Beyond the future consideration of generational and gender differences, it would also be valuable to consider the use of the proposed Ecological Transactional Model of Factors that Influence Referee Abuse within other sporting contexts. Rugby may differ from other American sports as it is founded on the traditions of Muscular Christianity (Watson, et al., 2005). Sports that do not have the same ties to religion or the values that coincide with this religious foundation may perceive referee abuse and the factors underlying abuse in different ways. Further, the historical foundations of rugby may differ from other American sports.

Finally, the researcher implores future research to be conducted with other sporting stakeholders. While this model suggested the factors that may influence the abuse behavior of any non-referee, it has been produced through the perceptions of coaches alone. Players, spectators, or administrators may understand referee abuse differently, potentially extending the model. Additionally, it would be interesting to note the ways in which referees perceive the factors presented in this model. Alignment among various stakeholder groups would help to increase the value of the model within a sporting context and would serve to enhance the trustworthiness of the model as it relates to abuse perceptions and potential solutions. This study is simply a starting point in understanding various perspectives of referee abuse in collegiate rugby in the United States. The presented model should be tested, extended, or, if necessary, reimagined. The value of understanding referee abuse in various contexts and through the lens of a myriad of stakeholders should not be overlooked. 


\section{Conclusion}

The current study expanded the literature on referee abuse. First, the study addressed the need for exploring referee abuse through the lens of a non-referee. Referees alone are unable to create a holistic picture of the phenomenon of referee abuse; thus, this study provided an opportunity to view a phenomenon such as referee abuse through a non-traditional lens. As seen in this study, utilizing a non-traditional stakeholder group to explore referee abuse has corroborated the perspectives of referees in numerous other studies. Further, studying referee abuse from the perspective of the coach has allowed for the consideration of factors that may underlie abuses. Exploring referee abuse from the perspective of the coach allows for investigation of the perpetrator's mentality, which ultimately opens up new avenues for exploration and, ideally, combating abuses.

Because this study was completed with the help of coaching participants, the factors that influence perceptions of abuse and abuse behaviors were discussed. These factors were categorized into the proposed Ecological Transactional Model of Factors that Influence Referee Abuse. This model and the factors that influence referee abuse that fit within it highlight the impact of one's ecology on their beliefs and behaviors. Understanding the foundations of referee abuse from a coach's perspective seemed to assist the coaches in developing solutions that may decrease such behaviors. These same notions could be applied to sport managers and administrators as they seek to deter referee abuse within their sporting structures. In essence, this study has found that it may be difficult to combat referee abuse without addressing the factors that underlie its existence. 
In addition to the proposal of the Ecological Transactional Model of Factors that Influence Referee Abuse, the findings in this study revealed coaches' understandings of referee abuse. Their understandings of referee abuse seem to mirror the understandings of referee abuse that exist in the literature, primarily derived from the referees. They found referee abuse to be challenging to define, prevalent in the rugby context, and marred by numerous negative consequences. The concurrence of these understandings between referees and coaches may prove invaluable in creating a unified approach to referee abuse.

Finally, this study proposed three avenues that sporting stakeholders may explore to combat referee abuse. The coaches in this study proposed that collaboration, education, and the formalization and enforcement of regulations may have a significant impact on referee abuse. Within each of these categorizations, the coaches proposed numerous strategies that may advent positive change. It is important to note that the coaches did not place all of the responsibility for such change on others. Rather, they suggested that coaches, too, had a role to play in the development and execution of solutions regarding referee abuse.

As this study was exploratory in nature, the outcomes represent the understandings of referee abuse from the perspective of one group of rugby coaches. Further, the proposed model serves as a first iteration of the ecological organization of factors that influence referee abuse. Future research can and should expand upon this model considering its fit with additional sports and varying stakeholders. In addition, future research may help to illuminate additional aspects of referee abuse that have yet to 
be uncovered. This exploratory research has led to the development of numerous additional inquiries and serves as a strong foundation for such research. 


\section{REFERENCES}

Ackery, A. D., Tator, C. H., \& Snider, C. (2012). Violence in Canadian amateur hockey: The experience of referees in Ontario. Clinical Journal of Sport Medicine, 22(2), 86-90.

Adler, N. J., Doktor, R., \& Redding, S. G. (1986). From the Atlantic to the Pacific century: Cross-cultural management reviewed. Journal of Management, 12, 295318.

Al-Emadi, A. A. Q., Schwabenland, C., \& Wei, Q. (2015). The vital role of employee retention in human resource management: A literature review. IUP Journal of Organizational Behavior, 14(3), 7-32.

Anderson, E. (2017, July 14). Soccer referee declared dead after being punched in game. USA Today. Retrieved from https://www.usatoday.com/story/sports/soccer/ 2014/07/01/soccer-referee-punched-john-bienewicz-dead/11906323/

Anshel, M. H., \& Weinberg, R. S. (1995). Sources of acute stress in American and Australian basketball referees. Journal of Applied Sport Psychology, 7(1), 11-22.

Anshel, M. H., \& Weinberg, R. S. (1996). Coping with acute stress among American and Australian basketball referees. Journal of Sport Behavior, 19(3), 180-203.

Anshel, M. H., Kang, M., \& Jubenville, C. (2013). Sources of acute sport stress scale for sports officials: Rasch calibration. Psychology of Sport and Exercise, 14(3), 362370. 
Armstrong, G. (1999). Football cultures and identities. New York: Springer.

Armstrong, D., Gosling, A., Weinman, J., \& Marteau, T. (1997). The place of inter-rater reliability in qualitative research: An empirical study. Sociology, 31(3), 597-606.

Arthur-Banning, S., Wells, M. S., Baker, B. L., \& Hegreness, R. (2009). Parents behaving badly? The relationship between the sportsmanship behaviors of adults and athletes in youth basketball games. Journal of Sport Behavior, 32(1), 3-18.

Ashforth, B. E., \& Mael, F. (1989). Social identity theory and the organization. Academy of Management Review, 14(1), 20-39.

Auger, D., Fortier, J., Thibault, A., \& Gravelle, F. (2010). Characteristics and motivations of sports officials in the province of Québec. International Journal of Sport Management, Recreation, \& Tourism, 5, 29-50.

Baldwin, C. (2008). The ref cost us the game: The role of sports commentators and journalists in creating stresses on sports referees. Power and Place, 7, 9-11.

Bandura, A. (1973). Aggression: A social learning analysis. Englewood Cliffs, NJ: Prentice-Hall.

Bandura, A., \& Walters, R. H. (1977). Social learning theory. Englewood Cliffs, NJ: Prentice-Hall.

Becker, H. S. (1986). Writing for social scientists: How to start and finish your thesis, book, or article. Chicago: University of Chicago Press.

Belsky, J. (1980). Child maltreatment: An ecological integration. American Psychologist, 35(4), 320-335.

Bertaux, D. (Ed.). (1981). Bibliography and society: The life history approach in the social sciences. London: Sage. 
Bloom, G. A., Durand-Bush, N., Schinke, R., \& Samela, J. H. (1998). The importance of mentoring in the development of coaches and athletes. International Journal of Sport Psychology, 29, 267-281.

Borsari, B., \& Carey, K. B. (2003). Descriptive and injunctive norms in college drinking: A meta-analytic integration. Journal of Studies on Alcohol, 64(3), 331-341.

Brackenridge, C. H. (1997a) 'He owned me basically...' Women's experiences of sexual abuse in sport. International Review for the Sociology of Sport 32(2): 115-130.

Brackenridge, C. H. (1997b). Sexual harassment and sexual abuse in sport. In Researching women and sport (pp. 126-141). London: Palgrave Macmillan. Brackenridge, C., Bringer, J. D., \& Bishopp, D. (2005). Managing cases of abuse in sport. Child Abuse Review, 14(4), 259-274.

Brackenridge, C., Fasting, K., Kirby, S., \& Leahy, T. (2010). Protecting children from violence in sport: A review with a focus on industrialized countries. Florence: UNICEF Innocenti Research Centre.

Brackenridge, C., Pitchford, A., \& Wilson, M. (2011). Respect: Results of a pilot project designed to improve behaviour in English football. Managing Leisure, 16(3), 175191.

Braun, V., \& Clarke, V. (2006). Using thematic analysis in psychology. Qualitative Research in Psychology, 3(2), 77-101.

Brennan, W. (2001). Dealing with verbal abuse. Emergency Nurse, 9(5), 15-17.

Britten, N. (1995). Qualitative interviews in medical research. The BMJ, 311, 251-253. 
Brockner, J., \& Wiesenfeld, B. M. (1996). An integrative framework for explaining reactions to decisions: Interactive effects of outcomes and procedures. Psychological Bulletin, 120(2), 189-208.

Bronfenbrenner, U. (1979). Contexts of child rearing: Problems and prospects. American Psychologist, 34(10), 844-850.

Burris, E. R., Detert, J. R., \& Romney, A. C. (2013). Speaking up vs. being heard: The disagreement around and outcomes of employee voice. Organization Science, 24(1), 22-38.

Caelli, K. (2000). The changing face of phenomenological research: Traditional and American phenomenology in nursing. Qualitative Health Research, 10(3), 366377.

Chiafullo, C. M. (1998). From personal foul to personal attack: How sports officials are the target of physical abuse from players, coaches and fans alike. Seton Hall Journal of Sport Law, 8, 201-225.

Chory-Assad, R. M., \& Paulsel, M. L. (2004). Classroom justice: Student aggression and resistance as reactions to perceived unfairness. Communication Education, 53(3) $253-273$.

Cikara, M., Bruneau, E., Van Bavel, J. J., \& Saxe, R. (2014). Their pain gives us pleasure: How intergroup dynamics shape empathic failures and counter-empathic responses. Journal of Experimental Social Psychology, 55, 110-125.

Cleland, J., O’Gorman, J., \& Bond, M. (2015). The English Football Association's Respect Campaign: The referees' view. International Journal of Sport Policy and Politics, 7(4), 551-563. 
Cicchetti, D., \& Lynch, M. (1993). Toward an ecological/transactional model of community violence and child maltreatment: Consequences for children's development. Psychiatry, 56(1), 96-118.

Collins, C. L., Fields, S. K., \& Comstock, R. D. (2008). When the rules of the game are broken: What proportion of high school sports-related injuries are related to illegal activity? Injury Prevention, 14, 34-48.

Collins, T. (2009). A social history of English rugby union. London: Routledge.

Colquitt, J. A., Greenberg, J., \& Zapata-Phelan, C. P. (2005). What is organizational justice? A historical overview. Handbook of Organizational Justice, 1, 3-58.

Colwell, S. (2000). The 'letter' and the 'spirit': Football laws and refereeing in the twenty-first century. Soccer \& Society, 1(1), 201-214.

Conroy, D. E., \& Coatsworth, J. D. (2006). Coach training as a strategy for promoting youth social development. The Sport Psychologist, 20(2), 128-144.

Creswell, J. W. (2013). Qualitative inquiry \& research design: Choosing among five approaches. Thousand Oaks, CA: Sage.

Crotty, M. (1998). The foundations of social research: Meaning and perspective in the research process. Thousand Oaks, CA: Sage.

Cuskelly, G., \& Hoye, R. (2013). Sports officials' intention to continue. Sport Management Review, 16(4), 451-464.

Cuskelly, G., Evans, G., \& Hoye, R. (2004). Problems and issues in the recruitment and retention of sports officials: A report prepared for the Australian Sports Commission. Queensland, Australia: Griffith Business School. 
Cuskelly, G., Hoye, R., \& Auld, C. (2006). Working with volunteers in sport: Theory and practice. New York: Routledge.

Dahlberg, K., Drew, N., \& Nystrom, M. (2008). Reflective lifeworld research (2nd ed.). Lund, Sweden: Studentlitteratur.

Dell, C., Gervis, M., \& Rhind, D. J. (2016). Factors influencing soccer referees' intentions to quit the game. Soccer \& Society, 17(1), 109-119.

Denzin, N. K. (1983). Interpretive interactionism. In G. Morgan (Ed.), Beyond method: Strategies for social research (pp. 129-146). Beverly Hills, CA: Sage.

De Vaus, D. (2014). Surveys in social research (6th ed.). New York: Routledge.

Division I-A Rugby. (n.d.). About Division I-A Rugby. Retrieved from http://d1 arugby.com/about-d1a/

Dodge, K. A., Dishion, T. J., \& Lansford, J. E. (Eds.). (2007). Deviant peer influences in programs for youth: Problems and solutions. New York: Guilford Press.

Dorsch, K. D., \& Paskevich, D. M. (2007). Stressful experiences among six certification levels of ice hockey officials. Psychology of Sport and Exercise, 8(4), 585-593.

Dressman, M. (2009). Using social theory in educational research: A practical guide. New York: Routledge.

Duda, J. L., Olson, L. K., \& Templin, T. J. (1991). The relationship of task and ego orientation to sportsmanship attitudes and the perceived legitimacy of injurious acts. Research Quarterly for Exercise and Sport, 62(1), 79-87.

Dunning, E., \& Sheard, K. (2005). Barbarians, gentlemen and players: A sociological study of the development of rugby football (2nd ed.). London: Routledge. 
Duxbury, J. (2002). An evaluation of staff and patient views of and strategies employed to manage inpatient aggression and violence on one mental health unit: A pluralistic design. Journal of Psychiatric and Mental Health Nursing, 9(3), 325337.

Evans, P. (2009). The verbally abusive relationship, expanded third edition: How to recognize it and how to respond. New York: Simon and Schuster.

Fields, S. K., Collins, C. L., \& Comstock, R. D. (2007). Conflict on the courts: A review of sports-related violence literature. Trauma, Violence, \& Abuse, 8(4), 359-369.

Finkelhor, D. (1984). Child sexual abuse. New theory \& research. New York: Free Press.

Finkelhor, D., \& Baron, L. (1986). Risk factors for child sexual abuse. Journal of Interpersonal Violence, 1(1), 43-71.

Finlay, L. (2003). Through the looking glass: Intersubjectivity and hermeneutic reflection. In L. Finlay, \& B. Gough (Eds.), Reflexivity: A practical guide for researchers in health and social sciences (pp. 105-119). Oxford: Blackwell Science.

Fischer, C. T. (2009). Bracketing in qualitative research: Conceptual and practical matters. Psychotherapy Research, 19(4-5), 583-590.

Folkesson, P., Nyberg, C., Archer, T., \& Norlander, T. (2002). Soccer referees' experience of threat and aggression: Effects of age, experience, and life orientation on outcome of coping strategy. Aggressive Behavior, 28(4), 317-327.

Folkman, S., \& Lazarus, R. S. (1985). If it changes it must be a process: Study of emotion and coping during three stages of college examination. Journal of Personality and Social Psychology, 48, 150-170. 
Fouche, F. (1993). Phenomenological theory of human science. In J. Snyman (Ed.), Conceptions of social inquiry (pp. 87-112). Cape Town, South Africa: HRSC Press.

Fraenkel, J. R., Wallen, N. E., \& Hyun, H. H. (2011). How to design and evaluate research in education (8th ed.). New York: McGraw-Hill.

Friman, M., Nyberg, C., \& Norlander, T. (2004). Threats and aggression directed at soccer referees: An empirical phenomenological psychological study. The Qualitative Report, 9(4), 652-672.

Fusch, P. I., \& Ness, L. R. (2015). Are we there yet? Data saturation in qualitative research. The Qualitative Report, 20(9), 1408-1416.

Gadamer, H. G. (1975). Hermeneutics and social science. Cultural hermeneutics, 2(4), 307-316.

Galletta, A. (2013). Mastering the semi-structured interview and beyond. New York: New York University Press.

Gardner, M., \& Steinberg, L. (2005). Peer influence on risk taking, risk preference, and risky decision making in adolescence and adulthood: An experimental study. Developmental Psychology, 41(4), 625-635.

Gencay, S. (2009). Magnitude of psychological stress reported by soccer referees. Social Behavior and Personality: An International Journal, 37(7), 865-868.

Glaser, B. G., \& Strauss, A. L. (1967). The discovery of grounded theory: Strategies for qualitative research. New York: Aldine de Gruyter.

Glaser, D. (2002). Emotional abuse and neglect (psychological maltreatment): A conceptual framework. Child Abuse \& Neglect, 26(6-7), 697-714. 
Go, M. H., Green, H. D., Kennedy, D. P., Pollard, M., \& Tucker, J. S. (2010). Peer influence and selection effects on adolescent smoking. Drug \& Alcohol Dependence, 109(1), 239-242.

Goldkuhl, G. (2012). Pragmatism vs interpretivism in qualitative information systems research. European Journal of Information Systems, 21(2), 135-146.

Goldsmith, P. A., \& Williams, J. M. (1992). Perceived stressors for football and volleyball officials from three rating levels. Journal of Sport Behavior, 15(2), 106-118.

Green, A. J., \& Holloway, D. G. (1997). Using a phenomenological research technique to examine student nurses' understandings of experiential teaching and learning: A critical review of methodological issues. Journal of Advanced Nursing, 26(5), 1013-1019.

Greenberg, J., \& Cropanzano, R. (2001). Advances in organizational justice. Stanford, CA: Stanford University Press.

Grimm, P. (2010, December). Social desirability bias. Wiley International Encyclopedia of Marketing. Retrieved from https://doi.org/10.1002/9781444316568.wiem02057

Guba, E. G., \& Lincoln, Y. S. (1994). Competing paradigms in qualitative research. Handbook of Qualitative Research, 2, 105-117.

Guest, G., Bunce, A., \& Johnson, L. (2006). How many interviews are enough? An experiment with data saturation and variability. Field Methods, 18(1), 59-82.

Guest, G., MacQueen, K. M., \& Namey, E. E. (2011). Applied thematic analysis. Thousand Oaks, CA: Sage. 
Hahn-Smith, S., \& Springer, F. (2005). Social norms theory. Prevention Tactics, 8(9), 16.

Hamlyn, D. W. (1995). Epistemology, history of. In T. Honderich (Ed.), The Oxford companion to philosophy (pp. 242-245). Oxford: Oxford University Press.

Harthill, M. (2009). The sexual abuse of boys in organized male sports. Men and Masculinities, 12(2), 225-249.

Haugaard, J. J. (2000). The challenge of defining child sexual abuse. American Psychologist, 55(9), 1036-1039.

Hays, D. G., \& Singh, A. A. (2012). Qualitative inquiry in clinical and educational settings. New York: The Guilford Press.

Hein, G. (1999). Is meaning making constructivism? Is constructivism meaning making? The Exhibitionist, 18(2), 15-18.

Henwood, K. L., \& Pidgeon, N. F. (1992). Qualitative research and psychological theorizing. British Journal of Psychology, 83(1), 97-111.

Hill, C. E., Knox, S., Thompson, B. J., Williams, E. N., Hess, S. A., \& Ladany, N. (2005). Consensual qualitative research: An update. Journal of Counseling Psychology, 52, 196-205.

Hill, C. E., Thompson, B. J., \& Williams, E. N. (1997). A guide to conducting consensual qualitative research. The Counseling Psychologist, 25, 517-572.

Hirschman, A. O. (1970). Exit, voice, and loyalty: Responses to decline in firms, organizations, and states. Cambridge, MA: Harvard University Press.

Hofstede, G. (1980). Culture's consequences: International differences in work-related values. Beverly Hills. CA: Sage. 
Huffman, R. C., \& Hegarty, W. H. (1993). Top management influence on innovations: Effects of executive characteristics and social culture. Journal of Management, 19(3), 549-574.

Hughes, T. (1895). Tom Brown's school days. Cambridge, MA: Houghton, Mifflin, \& Co.

Humphrey, J. A., \& Palmer, S. (2013). Deviant behavior: Patterns, sources, and control. Berlin: Springer Science \& Business Media.

Husman, B. F., \& Silva, J. M. (1984). Aggression in sport: Definitional and theoretical considerations. In J. M. Silva, \& R. S. Weinberg (Eds.), Psychological foundations of sport (pp. 246-260). Champaign, IL: Human Kinetics.

Husserl, S. (1946). Reflections on writing phenomenology. Human Studies, 18(1), 41-62.

Hycner, R. H. (1985). Some guidelines for the phenomenological analysis of interview data. Human studies, 8(3), 279-303.

Jacob, S. A., \& Furgerson, S. P. (2012). Writing interview protocols and conducting interviews for students new to the field of qualitative research. The Qualitative Report, 17(2), 1-10.

Johnson, G. (2009, February 10). Officially speaking. NCAA News. Retrieved from http://www.ncaa.com/news/ncaa/article/2009-02-10/officially-speaking

Jones, R. L., Armour, K. M., \& Potrac, P. (2002). Understanding the coaching process: A framework for social analysis. Quest, 54(1), 34-48.

Kafle, N. P. (2013). Lived experiences of middle level leaders in the Nepali institutional schools. Journal of Education and Research, 3(2), 59-76. 
Kaissidis-Rodafinos, A., \& Anshel, M. H. (2000). Psychological predictors of coping responses among Greek basketball referees. The Journal of Social Psychology, 140(3), 329-344.

Kaissidis-Rodafinos, A., Anshel, M. H., \& Porter, A. (1997). Personal and situational factors that predict coping strategies for acute stress among basketball referees. Journal of Sports Sciences, 15(4), 427-436.

Kassing, J. W., \& Barber, A. M. (2007). Being a good sport: An investigation of sportsmanship messages provided by youth soccer parents, officials, and coaches. Human Communication, 10(1), 61-68.

Keegan, R. J., Harwood, C. G., Spray, C. M., \& Lavallee, D. E. (2009). A qualitative investigation exploring the motivational climate in early career sports participants: Coach, parent and peer influences on sport motivation. Psychology of Sport and Exercise, 10(3), 361-372.

Total Youth Football. (2008). Keep it shut! The results. Total Youth Football, 5, 24-27. Kellett, P., \& Shilbury, D. (2007). Umpire participation: Is abuse really the issue? Sport Management Review, 10(3), 209-229.

Kellett, P., \& Warner, S. (2011). Creating communities that lead to retention: The social worlds and communities of umpires. European Sport Management Quarterly, 11, 471- 494.

Kelly, S., \& Waddington, I. (2006). Abuse, intimidation and violence as aspects of managerial control in professional soccer in Britain and Ireland. International Review for the Sociology of Sport, 41(2), 147-164. 
Kerka, S. (1998). New perspectives on mentoring. Retrieved from http://www.ericdigests.org/1998-3/ mentoring.html

Kerr, G., Stirling, A., \& MacPherson, E. (2014). A critical examination of child protection initiatives in sport contexts. Social Sciences, 3(4), 742-757.

Kim, S. (2017). Perceived organizational support as a mediator between distributive justice and sports referees' job satisfaction and career commitment. Annals of Leisure Research, 20(2), 169-187.

Kirschenbaum, D. S., \& Smith, R. J. (1983). A preliminary study of sequencing effects in simulated coach feedback. Journal of Sport Psychology, 5(3), 332-342.

Knight, M. (2009). Between the lines-the spirit of South African rugby. South Africa: Penguin.

Knox, S., \& Burkard, A. (2009). Qualitative research interviews. Psychotherapy Research, 19(4-5), 566-575.

Langdridge, D. (2007). Phenomenological psychology: Theory, research and method. London: Pearson Education.

Lazarus, R. S. (1991). Cognition and motivation in emotion. American Psychologist, 46(4), 352-367.

Lazarus, R. S. (1993). Coping theory and research: Past, present, and future. Psychosomatic Medicine, 55(3), 234-237.

LeBlanc, J. E., \& Dickson, L. (1997). Straight talk about children and sport: Advice for parents, coaches, and teachers. Oakville, Canada: Mosaic Press.

Liehr, P., \& Smith, M. J. (1999). Middle-range theory: Spinning research and practice to create knowledge for the new millennium. Advances in Nursing Science, 21(4), 8191. 
Lincoln, Y. S., \& Guba, E. G. (1985). Naturalistic inquiry (Vol. 75). Newbury Park, CA: Sage.

Livingston, L. A., \& Forbes, S. L. (2007). Factors contributing to the attrition of Canadian amateur ice hockey officials: Survey results from an Ontario-based district hockey association. Avante, 11(1), 1-14.

Loy, J. W., McPherson, B., \& Kenyon, G. (1978). Sport and social systems. Reading, MA: Addison-Wesley.

Malinowska, A., \& Lebek, K. (Eds.). (2016). Materiality and popular culture: The popular life of things (Vol. 92). New York: Taylor \& Francis.

Malley-Morrison, K., \& Hines, D. (2004). Family violence in a cultural perspective: Defining, understanding, and combating abuse. Thousand Oaks, CA: Sage.

Mangan, J. A., \& Walvin, J. (1987). Manliness and morality: Middle-class masculinity in Britain and America, 1800-1940. Oxford, England: Manchester University Press.

Mano, B. (2017, June). Taking care of us [Video file]. Retrieved from http://www.referee.com/pubmemo

Marcus, A. C., \& Crane, L. A. (1986). Telephone surveys in public health research. Medical Care, 24, 97-112.

Martens, M. P., Dams-O'Connor, K., \& Beck, N. C. (2006). A systematic review of college student-athlete drinking: Prevalence rates, sport-related factors, and interventions. Journal of Substance Abuse Treatment, 31(3), 305-316.

Mastroleo, N. R., Marzell, M., Turrisi, R., \& Borsari, B. (2012). Do coaches make a difference off the field? The examination of athletic coach influence on early college student drinking. Addiction Research \& Theory, 20(1), 64-71. 
Maxwell, J. A. (2008). Designing a qualitative study. The SAGE handbook of applied social research methods, 2, 214-253.

May, K. A. (1991). Interview techniques in qualitative research: Concerns and challenges. In J. M. Morse (Ed.), Qualitative nursing research: A contemporary dialogue (Rev. ed.). Thousand Oaks, CA: Sage.

May, C. R., Mair, F., Finch, T., MacFarlane, A., Dowrick, C., Treweek, S., ... \& Murray, E. (2009). Development of a theory of implementation and integration: Normalization process theory. Implementation Science, 4(1), 4-29.

Merriam, S. B., \& Tisdell, E. J. (2016). Qualitative research: A guide to design and implementation (4th ed.). San Francisco: Jossey-Bass.

Mertens, D. M. (2010). Research and evaluation in education and psychology: Integrating diversity with quantitative, qualitative, and mixed methods (3rd ed.). Thousand Oaks, CA: Sage.

Miles, M. B., \& Huberman, A. M. (1994). Qualitative data analysis-An expanded sourcebook. Newbury Park, CA: Sage.

Morse, J. M. (1994). Designing qualitative research. In N. K. Denzin \& Y. S. Lincoln (Eds.), Handbook of qualitative inquiry (pp. 220-235). Thousand Oaks, CA: Sage.

Moore, A. (2000). Opera of the proletariat: Rugby league, the labour movement and working-class culture in New South Wales and Queensland. Labour History, 79, 57-70.

Mountjoy, M., Brackenridge, C., Arrington, M., Blauwet, C., Carska-Sheppard, A., Fasting, K., ... \& Starr, K. (2016). International Olympic Committee consensus 
statement: Harassment and abuse (non-accidental violence) in sport. British Journal of Sports Medicine, 50(17), 1019-1029.

Moustakas, C. (1994). Phenomenological research methods. Thousand Oaks, CA: Sage. Musselwhite, K., Cuff, L., McGregor, L., \& King, K. M. (2006). The telephone interview is an effective method of data collection in clinical nursing research: A discussion paper. International Journal of Nursing Studies, 44, 1064-1070.

National Association for Sport Officials (2018). 2018 Sports Officials Legislative Scorecard. Retrieved from https://www.naso.org/resources/legislation/sportsofficials-legislative-scorecard/

Nazarudin, M. N., Omar-Fauzee, M. S., \& Din, A. (2009). Malaysian rugby referees' perception on the athletes, coaches, and spectator's misconduct: A case study. European Journal of Social Sciences, 7(4), 102-106.

Neave, N., \& Wolfson, S. (2003). Testosterone, territoriality, and the 'home advantage'. Physiology \& Behavior, 78(2), 269-275.

Nelson, K. H. (1980). The relationship between support for authority, support for sport officials, and disrespectful behavior in sport. Educational Resources Information Center (pp. 2-20). Retrieved from https://eric.ed.gov/?id=ED190496

Nevo, B. (1985). Face validity revisited. Journal of Educational Measurement, 22(4), 287-293.

O'Hagan, K. P. (1995). Emotional and psychological abuse: Problems of definition. Child Abuse \& Neglect, 19(4), 449-461. 
Oke, W. (2002). The recruitment and retention of soccer referees in New South Wales: NSW Soccer Federation Limited State Referees Report. Sydney: Soccer New South Wales.

Ortlipp, M. (2008). Keeping and using reflective journals in the qualitative research process. The Qualitative Report, 13(4), 695-705.

Palinkas, L. A., Horwitz, S. M., Green, C. A., Wisdom, J. P., Daun, N., \& Hoagwood, K. (2015). Purposeful sampling for qualitative data collection and mixed method implementation research. Administrative Policy Mental Health, 42(5), 533-544.

Pappas, D. (2016, October 18). The officiating crisis: What can I do? National Federation of State High School Associations. Retrieved from https://www.nfhs.org/articles/the-officiating-crisis-what-can-i-do/

Patrick, G. T. W. (1903). The psychology of football. The American Journal of Psychology, 14(3/4), 104-117.

Patton, M. Q. (1987). How to use qualitative methods in evaluation. London: Sage.

Perez, A. J. (2016, February 17). Soccer referee shot and killed on field by player he redcarded. USA Today. Retrieved from https://www.usatoday.com/story/sports/ soccer/2016/02/17/referee-shot-killed-red-card-soccer/80497628/

Perna, F. M., Zaichkowsky, L., \& Bocknek, G. (1996). The association of mentoring with psychosocial development among male athletes at termination of college career. Journal of Applied Sport Psychology, 8(1), 76-88.

Peters, K., \& Halcomb, E. (2015). Interviews in qualitative research. Nurse Researcher, $22(4), 6-7$. 
Phillips, P., \& Fairley, S. (2014). Umpiring: A serious leisure choice. Journal of Leisure Research, 46(2), 184-202.

Polkinghorne, D. E. (1989). Phenomenological research methods. In Existentialphenomenological perspectives in psychology (pp. 41-60). Boston: Springer.

Raakman, E., Dorsch, K., \& Rhind, D. (2010). The development of a typology of abusive coaching behaviours within youth sport. International Journal of Sports Science \& Coaching, 5(4), 503-515.

Rainey, D. (1995). Sources of stress among baseball and softball umpires. Journal of Applied Sport Psychology, 7(1), 1-10.

Rainey, D., \& Winterich, D. (1995). Magnitude of stress reported by basketball referees. Perceptual and Motor Skills, 81(3), 1241-1242.

Rainey, D. W., \& Duggan, P. (1998). Assaults on basketball referees: A statewide survey. Journal of Sport Behavior, 21(1), 113-120.

Rainey, D. W., \& Hardy, L. (1999). Sources of stress, burnout and intention to terminate among rugby union referees. Journal of Sports Sciences, 17(10), 797-806.

Ranby, K. W., Aiken, L. S., MacKinnon, D. P., Elliot, D. L., Moe, E. L., McGinnis, W., \& Goldberg, L. (2009). A mediation analysis of the ATHENA intervention for female athletes: Prevention of athletic-enhancing substance use and unhealthy weight loss behaviors. Journal of Pediatric Psychology, 34(10), 1069-1083.

Randolph, W., \& Viswanath, K. (2004). Lessons learned from public health mass media campaigns: Marketing health in a crowded media world. Annual Review of Public Health, 25, 419-437. 
Rayner, M., Webb, T., \& Webb, H. (2016). The occurrence of referee abuse in Rugby Union: Evidence and measures through an online survey. International Journal of Sport Management, Recreation and Tourism, 21, 66-81.

Ridinger, L. L. (2015). Contributors and constraints to involvement with youth sports officiating. Journal of Amateur Sport, 1(2), 103-127.

Ridinger, L. L., Kim, K. R., Warner, S., \& Tingle, J. K. (2017). Development of the Referee Retention Scale. Journal of Sport Management, 31(5), 514-527.

Rowe, M., \& Sherlock, H. (2005). Stress and verbal abuse in nursing: Do burned out nurses eat their young? Journal of Nursing Management, 13(3), 242-248.

Rudd, A. (2016). Do spectators have limits on their aggressive behaviors? Journal of Contemporary Athletics, 10(3), 177-184.

Rugby's Values. (2014, November 11). Retrieved from: https://www.worldrugby.org/ welcome-to-rugby/rugbys-values?lang=en

Ryan, G. W. (1999). Measuring the typicality of text: Using multiple coders for more than just reliability and validity checks. Human Organization, 58, 313-322.

Ryba, T. V., Schinke, R. J., \& Tenenbaum, G. (2010). The cultural turn in sport psychology. Morgantown, WV: Fitness Information Technology.

Sabaini, D. (2001). How to get \& keep officials. Special Report, The National Association of Sports Officials (pp. 1-41). Retrieved from https://www.docme.ru/ doc/410971/how-to-get-officials---national-association-of-sports-officials

SafeSport. (n.d.). Who we are. Retrieved from https://safesport.org/who-we-are 
Salvy, S. J., De La Haye, K., Bowker, J. C., \& Hermans, R. C. (2012). Influence of peers and friends on children's and adolescents' eating and activity behaviors. Physiology \& Behavior, 106(3), 369-378.

Sandelowski, M., \& Leeman, J. (2012). Writing usable qualitative health research findings. Qualitative Health Research, 22(10), 1404-1413.

Sapolsky, R. M. (2000). Stress hormones: Good and bad. Neurobiology of Disease, 7 , $540-542$.

Sarker, S. K. (1994). A critique of phenomenology. New Delhi, India: Atlantic Publishers. Schneider, S. C. (1989). Strategy formulation: The impact of national culture. Organization Studies, 10(2), 149-168.

Schwandt, T. A. (2015). The Sage dictionary of qualitative inquiry (4th ed.). Thousand Oaks, CA: Sage.

Scofield, D. E., \& Unruh, S. (2006). Dietary supplement use among adolescent athletes in central Nebraska and their sources of information. Journal of Strength and Conditioning Research, 20(2), 452-455.

Seitz, C. M., Wyrick, D. L., Rulison, K. L., Strack, R. W., \& Fearnow-Kenney, M. (2014). The association between coach and teammate injunctive norm reference groups and college student-athlete substance use. Journal of Alcohol and Drug Education, 58(2), 7-26.

Siemiatycki, J. (1979). A comparison of mail, telephone, and home interview strategies for household health surveys. American Journal of Public Health, 69, 238-245. 
Silk, M. (2005). Sporting ethnography: Philosophy, methodology \& reflection. In D. Andrews, D. Mason, \& M. Silk (Eds.), Qualitative methods in sports studies (pp. 65-103). Oxford, England: Berg.

Silva, J. M., \& Weinberg, R. S. (1984). Psychological foundations of sport. Champaign, IL: Human Kinetics.

Silverman, D. (1970). The theory of organizations: A sociological framework. London: Heineman Education.

Simmons, P. (2006). Tackling abuse of officials: Attitudes and communication skills of experienced football referees. Retrieved from https://www.adelaide.edu.au/anzca2006/conf_proceedings/simmons_peter_tacklin g_abuse_football_referees.pdf

Slabbert, A. D., \& Ukpere, W. I. (2010). A preliminary comparative study of rugby and football spectator attitudes towards violence. African Journal of Business Management, 4(4), 459-466.

Sokatch, A. (2006). Peer influences on the college-going decisions of low socioeconomic status urban youth. Education and Urban Society, 39(1), 128-146.

Solomon, A. (2014, October 27). USA Rugby announces new 10-year agreement with IMG. DI-A College Rugby News. Retrieved from http://d1arugby.com/news/usarugby-announces-new-10-year-agreement-with-img/

Sparkes, A. C., \& Smith, B. (2014). Qualitative research methods in sport, exercise and health: From process to product. New York: Routledge.

Sport Fitness Industry Association. (2016). Rugby participation report. Retrieved from https://www.sfia.org/reports/428_Rugby-Participation-Report-2016 
Sport Fitness Industry Association. (2016). Sports, fitness, and leisure activities topline participation report. Retrieved from https://www.sfia.org/reports/411_2016Sports\%2C-Fitness\%2C-and-Leisure-Activities-Topline-Participation-Report

Sport Fitness Industry Association. (2017). Rugby participation report. Retrieved from https://www.sfia.org/reports/557_Rugby-Participation-Report-2017

Sport Fitness Industry Association. (2017). Sports, fitness, and leisure activities topline participation report. Retrieved from https://www.sfia.org/reports/512_2017Sports\%2C-Fitness\%2C-and-Leisure-Activities-Topline-Participation-Report Sporting Goods Manufacturers Association. (2011). Sports, Fitness, and Recreational Activities Topline Participation Report. Retrieved from https://www.sfia.org/reports/276_2011-Sports\%2C-Fitness-\%26amp\%3BRecreational-Activities-Topline-Participation-Report

Stewart, D., \& Mickunas, A. (1990). Exploring phenomenology: A guide to the field and its literature. Athens, OH: Ohio University Press.

Stevenson, C. (2001). The third team: An inside look at officiating in ice hockey. Retrieved from http://www.canoe.ca/hockeyrefereeing/home.html

Stewart, M. J., \& Ellery, P. J. (1998). Sources and magnitude of perceived psychological stress in high school volleyball officials. Perceptual and Motor Skills, 87(3), $1275-1282$.

Stirling, A. E., \& Kerr, G. A. (2008). Defining and categorizing emotional abuse in sport. European Journal of Sport Science, 8(4), 173-181. 
Stirling, A. E., \& Kerr, G. A. (2013). The perceived effects of elite athletes' experiences of emotional abuse in the coach-athlete relationship. International Journal of Sport and Exercise Psychology, 11(1), 87-100.

Stirling, A. E., \& Kerr, G. A. (2014). Initiating and sustaining emotional abuse in the coach-athlete relationship: An ecological transactional model of vulnerability. Journal of Aggression, Maltreatment \& Trauma, 23(2), 116-135.

Strauss, A. (1987). Qualitative analysis for social scientists. Cambridge: Cambridge University Press.

Suri, H. (2011). Purposeful sampling in qualitative research synthesis. Qualitative Research Journal, 11(2), 63-75.

Tajfel, H. (1979). Individuals and groups in social psychology. British Journal of Clinical Psychology, 18(2), 183-190.

Tajfel, H., \& Turner, J. (2001). An integrative theory of intergroup conflict. In M. A. Hogg, \& D. Abrams (Eds.), Key readings in social psychology. Intergroup relations: Essential readings (pp. 94-109). New York: Psychology Press.

Tajfel, H., \& Turner, J. C. (1985). The social identity theory of intergroup behavior. In S. Worchel, \& W. G. Austin (Eds.), Psychology of intergroup relations. (2nd ed.) (pp. 7-24). Chicago: Nelson-Hall.

Taylor, A. H., Daniel, J. V., Leith, L., \& Burke, R. J. (1990). Perceived stress, psychological burnout and paths to turnover intentions among sport officials. Journal of Applied Sport Psychology, 2(1), 84-97.

Turner, J. C., Brown, R. J., \& Tajfel, H. (1979). Social comparison and group interest in ingroup favouritism. European Journal of Social Psychology, 9(2), 187-204. 
Tenenbaum, G., Singer, R. N., Stewart, E., \& Duda, J. (1997). Aggression and violence in sport: An ISSP position stand. The Sport Psychologist, 11(1), 1-7.

Terrell, A. (2017, April 29). Shocking moment rugby league referee is knocked-out with a huge punch by player. The Sun. Retrieved from https://www.thesun.co.uk/ sport/rugbyleague/3436519/rugby-league-referee-knocked-out-punch/

Topp, B. (2001, May). Sports officials becoming endangered species. The National Association of Sports Officials. Retrieved from http://www.naso.org/ Research/st80.htm

Top Quartile Performance Institute. (2016). About strategy 2020. Retrieved from https://www.rugbystrategy.us/

Trucco, E. M., Colder, C. R., \& Wieczorek, W. F. (2011). Vulnerability to peer influence: A moderated mediation study of early adolescent alcohol use initiation. Addictive Behaviors, 36(7), 729-736.

Turner, D. W. (2010). Qualitative interview design: A practical guide for novice investigators. The Qualitative Report, 15(3), 754-760.

USA Rugby. (n.d.). About USA Rugby. Retrieved from https://www.usarugby.org/aboutusa-rugby/

USA Rugby Coaches. (n.d.). USA Rugby. Retrieved from https://www.usarugby.org/ coaching-requirements/

Van den Bos, K., Vermunt, R., \& Wilke, H. A. (1997). Procedural and distributive justice: What is fair depends more on what comes first than on what comes next. Journal of Personality and Social Psychology, 72(1), 95-104. 
Van Manen, M. (1990). Beyond assumptions: Shifting the limits of action research. Theory into Practice, 29(3), 152-157.

Van Manen, M. (Ed.). (2003). Writing in the dark: Phenomenological studies in interpretive inquiry. Walnut Creek, CA: Left Coast Press.

Vella, S., Oades, L., \& Crowe, T. (2011). The role of the coach in facilitating positive youth development: Moving from theory to practice. Journal of Applied Sport Psychology, 23(1), 33-48.

Voight, M. (2009). Sources of stress and coping strategies of US soccer officials. Stress and Health, 25(1), 91-101.

Vitaro, F., Brendgen, M., Ladouceur, R., \& Tremblay, R. E. (2001). Gambling, delinquency, and drug use during adolescence: Mutual influences and common risk factors. Journal of Gambling Studies, 17(3), 171-190.

Walters, S., Schluter, P., Stamp, D., Thomson, R., \& Payne, D. (2016). Coaches and referees' perspectives of sideline behaviour in children's team sports: a crosssectional study. International Journal of Sport Management, Recreation, and Tourism, 23, 51-74.

Walton, G. M. (1992). Beyond wining: The timeless wisdom of great philosopher coaches. Champaign, IL: Human Kinetics.

Warner, S., Tingle, J. K., \& Kellett, P. (2012). An administrative mess: A case study from the officiating community. Sport Management Review, 15(3), 368-380.

Warner, S., Tingle, J. K., \& Kellett, P. (2013). Officiating attrition: The experiences of former referees via a sport development lens. Journal of Sport Management, 27(4), 316-328. 
Watson, N. J., Weir, S., \& Friend, S. (2005). The development of Muscular Christianity in Victorian Britain and beyond. Journal of Religion and Society, 7, 1-21.

Webb, T., Rayner, M., \& Thelwell, R. (2018). An explorative investigation of referee abuse in English Rugby League. Journal of Applied Sport Management, 10(2), $14-25$.

Weinberg, R. S., \& Richardson, P. A. (1990). Psychology of officiating. Champaign, IL: Leisure Press.

Weiss, J. A., \& Tschirhart, M. (1994). Public information campaigns as policy instruments. Journal of Policy Analysis and Management, 13(1), 82-119.

Whittle, H., Hamilton-Giachritsis, C., Beech, A., \& Collings, G. (2013). A review of young people's vulnerabilities to online grooming. Aggression and Violent Behavior, 18(1), 135-146.

Wiederman, M. W. (1999). Volunteer bias in sexuality research using college student participants. Journal of Sex Research, 36(1), 59-66.

Wolfson, S., \& Neave, N. (2007). Coping under pressure: Cognitive strategies for maintaining confidence among soccer referees. Journal of Sport Behavior, 30(2), 232-247.

Woods, R. B. (2007). Social issues in sport. Chicago: Human Kinetics.

World Rugby. (2014, November 16). World Rugby Vision. Retrieved from https://www.worldrugby.org/vision?lang=en

World Rugby. (2017). Laws of the game. Dublin: World Rugby.

World Rugby. (n.d.). A beginner's guide to Rugby union. Retrieved from https://passport.worldrugby.org/?page=beginners \&p=7 
WSF. (2016, July 19). Addressing the issue of verbal, physical and psychological abuse of athletes: The foundation position. Retrieved from https://www.womenssports foundation.org/advocate/title-ix-and-issues/title-ix-positions/addressing-issueverbal-physical-psychological-abuse-athletes-foundation-position/

Yendle, C., Leverton, J., Kemp, J., \& Batts, F. (2007). Brief history of soccer referees. Retrieved from http://www.gdsra.com.au/history.htm

Zoller, S. (1985). Learning how to live with stress. Referee Magazine, 10(5), 48-51. 


\section{APPENDICES}

Appendix A: Demographic Questionnaire \& Consent Form

Project Title: Exploring Referee Abuse through the Lens of the Collegiate Rugby Coach Researcher: Brittany Jacobs (brittany.jacobs@unco.edu)

Research Advisors: Dr. Mark Smith \& Dr. Brent Oja

Email: Mark.Smith@unco.edu; Brent.Oja@unco.edu

This research study aims to better understand referee abuse in the rugby sporting structure. This information can help sport managers to consider referee abuse holistically, rather than utilizing only the perceptions of referees. It also may provide the opportunity for practical solutions regarding referee abuse. This study will seek to fill a gap in the existing literature, as no previous research has addressed referee abuse from a non-referee perspective.

By completing and returning this questionnaire, you are agreeing to participate in this study. You may be solicited to participate in future one-on-one, audio recorded interviews where you will be invited to share your experiences and perceptions. Your experiences, along with other members of this study, will be transcribed verbatim by the investigator and analyzed to develop several themes describing what you have shared regarding referee abuse.

No compensation will be provided to participants in this study. Foreseeable risks are not greater than those that might be encountered in a classroom environment or a conversation with a colleague about one's rugby experiences.

Participation is voluntary. You may decide not to participate in this study at any time. Even if you have begun participation, you may still decide to stop and withdraw at any time. Your decision will be respected and will not result in loss of benefits to which you are otherwise entitled. Having read the above information and having had an opportunity to ask any questions, please proceed with the pre-screening questionnaire if you would like to participate in this research. By completing the questionnaire, you are providing your permission for participation. You may keep this form for future reference.

If you have any concerns about your selection or treatment as a research participant, please contact Sherry May, IRB Administrator, Office of Sponsored Programs, 25 Kepner Hall, University of Northern Colorado Greeley, CO 80639; 970-351-1910

1. Name

2. Are you currently registered (CIPPed) as a coach with USA Rugby?

3. Are you currently a men's DI collegiate rugby coach?

4. Have you ever witnessed referee abuse within the sport of rugby? [Referee abuse is defined as a verbal statement or physical act which implies or threatens physical harm to a referee or a referee's property or equipment. Abuse includes, 
but is not limited to, the following acts committed on a referee: using foul or abusive language toward a referee; spewing any beverage on a referee's personal property; or verbally threatening a referee....(Chiafullo, 1998, p. 202)]

5. How many years have you coached men's Division I-A rugby?

6. How many years have you coached rugby at any level (including Division I-A rugby)?

7. Would you be willing to complete two interviews regarding officiating abuse within the rugby sporting context? The interviews will each take approximately one hour and may be scheduled at your convenience during the months of March and April. All information provided during this interview will be kept confidential and will not be associated with your name.

a. If you are willing to participate, please provide the following information:

i. Email address

ii. Phone number 
Appendix B: Institutional Review Board Approval

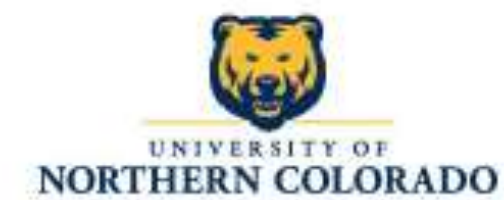

\section{Institutional Review Board}

DATE:

March 6, 2018

TO:

Brittany Jacobs

FROM:

University of Northem Colorado (UNCO) IRB

PROJECT TITLE:

[1205875-1] Exploring Referee Abuse Through the Lens of the Collegiate Rugby Coach

SUBMISSION TYPE:

New Project

ACTION:

APPROVALVERIFICATION OF EXEMPT STATUS

DECISION DATE:

March 6, 2018

EXPIRATION DATE:

March 6, 2022

Thank you for your submission of New Project materials for this project. The University of Northem Colorado (UNCO) IRB approves this project and verifies its status as EXEMPT according to federal IRB regulations.

Brittany -

Thank you for a thorough and clear IRB application for your dissertation research. All protocols and materials are approved and you may begin participant recruitment and data collection.

Best wishes with your study. Please don't hesitate to contact me with any IRB-related questions or concerns.

Sincerely.

Dr. Megan Stellino, UNC IRB Co-Chair

We will retain a copy of this correspondence within our records for a duration of 4 years.

If you have any questions, please contact Sherry May at 970-351-1910 or Sherry.Mayqunco.edu. Please include your project title and reference number in all correspondence with this committee.

This ietter has been eiectronicalty signed in accordance with al applicable regulations, and a copy la retained wthin University of Northern Colorado (UNCO) IRB's recorda. 


\section{Appendix C: Participation Solicitation Email to Coaches}

\section{Dear USA Rugby Coaches,}

My name is Brittany Jacobs and I am currently a doctoral candidate in the School of Sport \& Exercise Science at the University of Northern Colorado. As part of my dissertation, I am conducting a study regarding perceptions of referee abuse in rugby from the perspective of the rugby coach. I would like to invite you to participate in this study! Your insights will be incredibly helpful, and your participation would be much appreciated.

The study will be qualitative in nature, and should you be interested in participating, I would ask that you complete two 40 - 60 minute phone interviews. These interviews can be conducted at your convenience. Your participation is vitally important to this study and will seek to broaden the understanding of referee abuse with the rugby sporting structure.

If you are interested in participating in this study, please complete this brief survey in order to determine your eligibility for the study. Thank you, in advance, for your consideration and please feel free to contact me at Brittany.Jacobs@unco.edu with any questions or concerns!

Thank you,

Brittany Jacobs, M.Ed.

Brittany.Jacobs@unco.edu

School of Sport \& Exercise Science

The University of Northern Colorado 
Appendix D: Screening Questionnaire

Q1 Project Title: Exploring Referee Abuse through the Lens of the Collegiate Rugby Coach Researcher: Brittany Jacobs (brittany.jacobs@unco.edu)

Research Advisors: Dr. Mark Smith \& Dr. Brent Oja

Email: Mark.Smith@unco.edu; Brent.Oja@ unco.edu

This research study aims to better understand referee abuse in the rugby sporting structure. This information can help sport managers to consider referee abuse holistically, rather than utilizing only the perceptions of referees. It also may provide the opportunity for practical solutions regarding referee abuse. This study will seek to fill a gap in the existing literature, as no previous research has addressed referee abuse from a non-referee perspective.

The following screening questionnaire should take approximately 5 minutes to complete. Should you meet the requisite criteria for the study, you may be solicited to participate in one-on-one, audio recorded interviews where you will be invited to share your experiences and perceptions. Interviewees will be provided a pseudonym to protect the confidentiality of their responses. Your experiences, along with other members of this study, will be transcribed verbatim by the investigator and analyzed to develop several themes describing what you have shared regarding referee abuse.

No compensation will be provided to participants in this study. Foreseeable risks are not greater than those that might be encountered in a classroom environment or a conversation with a colleague about one's rugby experiences.

Participation is voluntary. You may decide not to participate in this study at any time. Even if you have begun participation, you may still decide to stop and withdraw at any time. Your decision will be respected and will not result in loss of benefits to which you are otherwise entitled. Having read the above information, please proceed with the prescreening questionnaire if you would like to participate in this research. By completing the questionnaire, you will be screened for participation and are ultimately providing your permission for participation. You may keep this form for future reference. If you would not like to participate, please close your browser window at this time.

If you have any concerns about your selection or treatment as a research participant, please contact Sherry May, IRB Administrator, Office of Sponsored Programs, 25 Kepner Hall, University of Northern Colorado Greeley, CO 80639; 970-351-1910

I agree to complete the screening questionnaire (1)

Page Break 
Q2 What is your full name?

Q3 What is the best email address to contact you in regard to this study?

Q4 What is the best phone number to reach you for this study?

Q6 Are you currently registered (CIPPed) as a coach with USA Rugby?

Yes (1)

No (2)

End of Block: Default Question Block

Start of Block: Block 3

Q7 Are you currently a men's DI-A collegiate rugby coach?

Yes (1)

No (2)

End of Block: Block 3

Start of Block: Block 2

Q5 Have you ever witnessed referee abuse in rugby? 
[Referee abuse is defined as: "a verbal statement or physical act which implies or threatens physical harm to a referee or a referee's property or equipment. Abuse includes, but is not limited to, the following acts committed on a referee: using foul or abusive language toward a referee; spewing any beverage on a referee's personal property; or verbally threatening a referee..."(Chiafullo, 1998, p. 202)

Yes (1)

No (2)

\section{End of Block: Block 2}

\section{Start of Block: Block 1}

Q8 How many years have you coached men's DI-A collegiate rugby?

$$
\begin{array}{lllllllllll}
0 & 4 & 8 & 12 & 16 & 20 & 24 & 28 & 32 & 36 & 40
\end{array}
$$

Years coaching men's DI-A collegiate

rugby ()

Q10 How many years have you coached rugby (at any level)?

Years coaching rugby ()

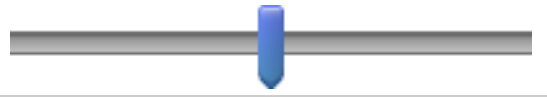

Q11 1. Are you willing to complete two interviews regarding referee abuse in rugby? The interviews will each take approximately one hour and may be scheduled at your convenience. Interviews will commence on March 20th. All information provided during these interviews will be kept confidential and will not be associated with your name.

Yes, I would like to participate in the study (1)

No, I would not like to participate in this study (2) 
End of Block: Block 1

Start of Block: Block 4

Q12 Thank you for completing this brief questionnaire. You have qualified to participate in this study. Are you prepared to schedule your first interview at this time?

Yes, please allow me to schedule my first interview (1)

No, please contact me to schedule my first interview (2)

End of Block: Block 4 
Appendix E: Consent Form

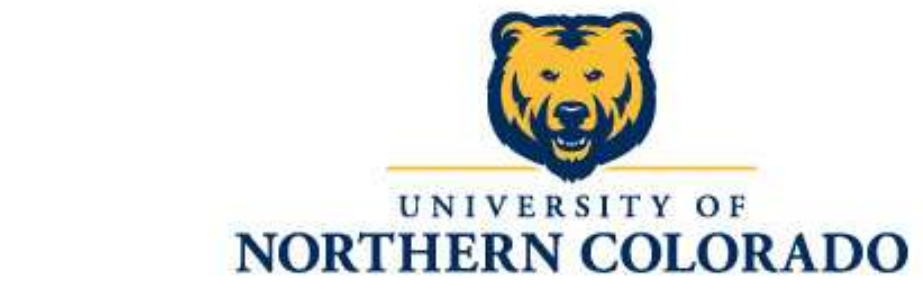

\title{
CONSENT FORM FOR HUMAN PARTICIPANTS IN RESEARCH UNIVERSITY OF NORTHERN COLORADO
}

\author{
Project Title: Exploring Referee Abuse through the Lens of the Collegiate Rugby Coach \\ Researcher: Brittany Jacobs (brittany.jacobs@unco.edu) \\ Research Advisors: Dr. Mark Smith \& Dr. Brent Oja \\ Email: Mark.Smith@unco.edu; Brent.Oja@unco.edu
}

This research study seeks to better understand referee abuse in the rugby sporting structure. This information can help sport managers to consider referee abuse holistically, rather than utilizing only the perceptions of referees. It also may provide the opportunity for practical solutions regarding referee abuse. This study will seek to fill a gap in the existing literature, as no previous research has addressed referee abuse from a non-referee perspective.

Through participating in this one-on-one, audio recorded, interview, you will be invited to share your experiences and perceptions. Your experiences, along with other members of this study, will be transcribed verbatim by the investigator and analyzed to develop several themes describing what you have shared regarding referee abuse. We estimate that the two interview sessions will each take between forty and sixty minutes to complete. You may also be contacted for further clarification in confirming the accuracy of the interview transcripts. Your personal information will be altered as we will assign a pseudonym for you, and only the local investigators will know the name connected with a pseudonym. Data collected and analyzed for this study will be kept in a password-protected file in each investigator's office. Only pseudonyms will be used to report data and the data will be destroyed three years after collection.

The cost for participating in this study is that of the time invested in participating in the interview. No compensation will be provided to participants in this study. Foreseeable risks are not greater than those that might be encountered in a classroom environment or a conversation with a colleague about one's rugby experiences.

Participation is voluntary. You may decide not to participate in this study at any time. Even if you have begun participation, you may still decide to stop and withdraw at any time. Your decision will be respected and will not result in loss of benefits to which you are otherwise entitled. Having read the above information and having had an opportunity to ask any questions, please proceed with the interview if you would like to participate in this research. By completing the interview, you are providing your permission for participation. You may keep this form for future reference.

If you have any concerns about your selection or treatment as a research participant, please contact Sherry May, IRB Administrator, Office of Sponsored Programs, 25 Kepner Hall, University of Northern Colorado Greeley, CO 80639; 970-351-1910 
Appendix F: Interview Guide I

Date:

Interview Start Time:

Interview Completion Time:

Interviewee:

Pseudonym for Interviewee:

Interviewee Contact Phone number:

\section{Introduction:}

Hi! I'm Brittany Jacobs, a third-year doctoral student in Sport Administration at the University of Northern Colorado. I am currently working on a research project that seeks to explore coaches' perceptions of referee abuse and potential solutions. As such, this interview will include questions related to your perceptions and experiences with referee abuse in the rugby-sporting context. Please note that there are no right or wrong answers and that you will be provided a pseudonym so that all of your responses remain anonymous.

\section{Distribute and explain the informed consent and anonymity}

- Review the consent form with the participant

- Solicit verbal acceptance of the consent form

- Provide the participant with an emailed copy of the informed consent for their records

Interview Questions: The following questions are representative of the questions that will be asked during the interviews. Because the interviews are semi-structured additional topics may be explored, as well.

1. Rugby background
a. Experience
b. Roles
c. How you got involved

2. In your opinion, what actions constitute referee abuse?

3. For this study we will define referee abuse as: "a verbal statement or physical act which implies or threatens physical harm to a referee or a referee's property or equipment. Abuse includes, but is not limited to, the following 
acts committed [against] a referee: using foul or abusive language toward a referee; spewing any beverage on a referee's personal property; or verbally threatening a referee" (Chiafullo, 1998, p. 202). Based on this definition can you, please, describe any instances of referee abuse that you have seen or experienced during your time in rugby?

a. How do you see the referee abuse impacting the sport of rugby, if at all?

b. Can you describe the most egregious act of referee abuse that you have seen?

c. What types of referee abuse do you see most commonly? (verbal, physical?)

3. Who do you feel is responsible for the majority of referee abuse in rugby? (Players, coaches, spectators, etc.)

a. What have you seen or experienced that makes you believe this group is primarily responsible for referee abuse?

4. Why do you think that people participate in referee abuse?

5. What factors (contexts or situations) have influenced or affected your experiences with referee abuse?

a. Other's likelihood to engage in referee abuse?

b. Does the ___ impact one's likelihood to abuse a referee?

i. Personal characteristics?

1. Previous exposure to abuses

2. Current/previous roles 


\section{Coaching philosophy}

ii. Peers

iii. Collegiate rugby community? USA Rugby community? Coaching community?

iv. US sporting culture (beyond rugby?)

v. International sporting culture - history of rugby?

vi. Situational factors

1. quality of the referee

2. the caliber of the competition

3. the formality of the match

6. Do you think that there are situations in which referee abuse is warranted or justified? If so, please describe these situations.

7. If you were writing the definition of 'referee abuse' for rugby in the United states, how would you define referee abuse?

8. What do you think that coaches can do to help with the issue of referee abuse?

a. Are there any other solutions would you suggest to help mitigate the problem of referee abuse?

\section{Conclusion:}

- Thank you for your participation in this study - your input will be invaluable!

- Again, we will use a pseudonym when using any of your responses in order to increase your confidentiality

- If you have any questions or concerns, or would like to remove yourself from this study, please feel free to contact me at any time. 
Appendix G: Interview Guide II

Date: Interview II Start Time: Interview II Completion

Time:

Interviewee:

Pseudonym for Interviewee:

Interviewee Contact Phone number:

Date of completion of Interview I:

Interview Questions: The following questions are representative of the questions that will be asked during the interviews. Because the interviews are semi-structured additional topics may be explored, as well.

Reminder: Please note that there are no right or wrong answers and that you have been provided a pseudonym so that all of your responses remain anonymous.

\section{Introduction:}

Hi! I hope that all has been well since our last chat. Since our previous chat are there any concerns or additional thoughts that you would like to add at this time?

\section{Understanding ref abuse}

1. Current definition- 'behavior which implies or threatens physical harm'

a. Should abuse definition only include things that imply or threaten physical harm? If no, what else?

b. Many used the terms 'threaten or intimidate' to describe abuse - what does this mean to you?

c. What behaviors would be threatening or intimidating?

2. Perceptions of impact of ref abuse on:
a. Sporting stakeholders
b. Match
c. Community
d. Sport itself

3. Intervention in referee abuse:

a. When/why do you interject?

b. What actions do you take as a coach? Are those actions dependent on the type of abuse?

c. Are you seeing others self-policing? What actions are they taking? 
d. Does referee abuse reflect negatively on the coach/competition? Why/why not?

\section{Factors Impacting Referee Abuse}

1. Understanding Rugby Culture
a. What is American Sporting Culture
b. What is rugby sporting culture?
c. Do they align?
d. How do you teach/establish 'rugby culture'?

2. US vs. Them

a. Are you seeing an us vs. them mentality between referees and coaches?

b. What factors impact the us vs. them mentality?

c. Do coaches/refs regularly interact?

d. Power struggle?

3. Professionalization of rugby vs. amateur rugby impact on referee abuse

a. Win at all costs?

4. Safety?

5. Fairness?

Solutions

1. How do we remedy the issues listed above?
a. Us vs. them
b. Lack of safety/fairness
c. Cultural issues

2. Continuing Education noted as a good solution - what do you want to see in these programs?

3. What are the impediments to enacting the previous solutions put forth?
a. Education
b. Collaboration
c. All coaches certified as refs, as many players as possible
d. Managing appointments

\section{Conclusion:}

- Next steps - send transcript for review

- Thank you for your participation in this study - your input has been invaluable!

- Again, we will use your pseudonym when using any of your responses in order to increase your anonymity

- If you have any questions or concerns, or would like to remove yourself from this study, please feel free to contact me at any time 